\title{
Operator Algebras and Conformal Field Theory
}

\author{
Fabrizio Gabbiani and Jürg Fröhlich \\ Institut für Theoretische Physik, ETH-Hönggerberg, CH-8093 Zürich, Switzerland
}

Received October 7, 1992; in revised form February 19, 1993

\begin{abstract}
We define and study two-dimensional, chiral conformal field theory by the methods of algebraic field theory. We start by characterizing the vacuum sectors of such theories and show that, under very general hypotheses, their algebras of local observables are isomorphic to the unique hyperfinite type III $_{1}$ factor. The conformal net determined by the algebras of local observables is proven to satisfy Haag duality. The representation of the Moebius group (and presumably of the entire Virasoro algebra) on the vacuum sector of a conformal field theory is uniquely determined by the Tomita-Takesaki modular operators associated with its vacuum state and its conformal net. We then develop the theory of Moebius covariant representations of a conformal net, using methods of Doplicher, Haag and Roberts. We apply our results to the representation theory of loop groups. Our analysis is motivated by the desire to find a "background-independent" formulation of conformal field theories.
\end{abstract}

\section{Contents}

I. Introduction . . . . . . . . . . . . . . . . . . . . . . . . . . . 570

II. Structure of the vacuum representation of a conformal field theory $\quad$. . . . . . . . . 574

II.1 Conformal nets of operator algebras . . . . . . . . . . . . . . . . . . 574

II.2 Moebius covariance and Tomita-Takesaki modular operators; Haag duality . . . 581

II.3 Local internal symmetries of vacuum sectors . . . . . . . . . . . . . . . 587

III. The conformal nets associated to positive-energy representations of loop groups . . . 591

III.1 Simple, simply laced Lie algebras and groups . . . . . . . . . . . . . . . 591

III.2 The affine Lie algebra $\hat{\mathscr{G}}$ associated with $\mathscr{G}$. . . . . . . . . . . . . . . 592

III.3 The loop group $L G_{0}$ and its central extension $\widehat{L G}_{0} \quad \ldots . . . \quad . \quad . \quad . \quad . \quad . \quad . \quad . \quad 593$

III.4 The local structure of $L G_{0}$ and $\widehat{L G}_{0} \quad$. . . . . . . . . . . . . . . . . . . . . 595

III.5 The extension of $\hat{\mathscr{G}}$ by the Virasoro algebra _. . . . . . . . . . . . . . 596

III.6 The root space decomposition of $\hat{\mathscr{G}}$; dominant integral weights . . . . . . . 597

III.7 Properties of irreducible integrable highest weight modules of $\hat{\mathscr{G}}^{e} \quad$. . . . . . 598

\footnotetext{
$\star$ Typeset using $\mathrm{T}_{\mathrm{E} X}$
} 
III.8 Vacuum representations of $\widehat{L G}_{0}$; the basic representation _ . . . . . . . . . 599

III.9 Local algebras in irreducible representation of $\widehat{L G}_{0}$. . . . . . . . . . . . 600

IV. Representation theory of conformal nets . . . . . . . . . . . . . . . . . . . 601

IV.1 Definitions, basic properties . . . . . . . . . . . . . . . . . . . . . . 601

IV.2 Composition of sectors . . . . . . . . . . . . . . . . . . . . . . . . 604

IV.3 Haag-Kastler subnets on light-rays . . . . . . . . . . . . . . . . . . . 610

IV.4 Braid statistics operators . . . . . . . . . . . . . . . . . . . . . . . 614

IV.5 Superselection sectors with finite statistical dimension . . . . . . . . . . . 616

IV.6 Superselection structure of conformal nets constructed from vacuum representations of loop groups . . . . . . . . . . . . . . . . . . . . . . . . . . . . . . 626

Appendix I. Notation and Geometry . . . . . . . . . . . . . . . . . . . . . . 628

Appendix II. Proof of Lemma 2.22 . . . . . . . . . . . . . . . . . . . . . . . . . 633

Appendix III. Proof of Theorem 4.42 . . . . . . . . . . . . . . . . . . . . . . 636

Acknowledgments . . . . . . . . . . . . . . . . . . . . . . . . . . . . . . 637

References . . . . . . . . . . . . . . . . . . . . . 638

\section{Introduction}

Eight years ago, the seminal paper of Belavin, Polyakov and Zamolodchikov [1] triggered renewed interest among theoretical physicists in two-dimensional conformal field theories and their infinite-dimensional symmetry algebras (the Virasoro algebra, Kac-Moody algebras and $W$-algebras). An impressive body of knowledge concerning conformal field theories has since been accumulated. Two-dimensional conformal field theories have been found to be important tools in the theoretical analysis of many physical systems. Among such systems are two-dimensional statistical systems at a critical point (theory of critical phenomena) [2], systems of condensed matter physics, such as quantum Hall fluids [3], polymers [4], Kondo systems [5]. Quite generally, physical systems exhibiting critical phenomena involving infinitely many degrees of freedom which - possibly after dimensional reduction - form a subsystem in a twodimensional space-time can be studied with the help of techniques from conformal field theory.

Perhaps the deepest applications of these theories have been made in the context of string theory [6]. Conformal field theory is to string theory what the theory of irreducible, unitary representations of the Poincare group is to relativistic quantum field theory. A classification of conformal field theories would therefore appear to represent an important issue. Much work has, in fact, been devoted to this problem.

One of the fascinating aspects of conformal field theories is that their analysis involves a wide range of fairly sophisticated mathematical tools: the theory of infinitedimensional Lie algebras and loop groups [7], algebraic geometry [8], the theory of tensor categories [9], operator algebra theory, in particular Jones' theory of inclusion of subfactors [10], BRST cohomology and quantum group theory [11], etc ...

Algebraic quantum field theory, founded by $\mathrm{R}$. Haag in 1955, is a precise mathematical formulation of quantum systems with infinitely many degrees of freedom in the language of the theory of $C^{*}$ - and von Neumann algebras. For a survey of results and methods and an account of the history see [12] (and refs. given there). Algebraic quantum field theory enables one to study structural features of the quantum theory of systems with infinitely many degrees of freedom in a general and mathematically clean way independent of special models. Among general properties of such systems studied with the methods of algebraic quantum field theory are: the classification of parastatistics [13, 14], the localization properties of charged fields in quantum field theories with a non-vanishing mass gap [15], quantum electrodynamics and 
scattering theory of infraparticles [16], the quantum-mechanical Noether theorem [17], a general formulation of the Goldstone theorem [18], etc ... Most important, the algebraic formulation of quantum field theory provides a precise mathematical description of superselection rules and the conserved charges associated with them (e.g., the electric charge, the baryon number, isospin, ...) [19], although it does not directly elucidate their physical origin. Superselection sectors are interpreted as Hilbert spaces of states carrying inequivalent representations of a net of algebras of local "observables". Superselection sectors can be composed in a way analogous to taking the tensor product of two representations (or the corresponding representation spaces) of a compact group. Mathematically, they can be viewed as the objects of a tensor category [20,10]. Recently, Doplicher and Roberts have brought the general theory of superselection rules and quantum statistics to a high degree of perfection by showing that the tensor categories of superselection sectors in four- or higher dimensional quantum field theories are isomorphic to categories of representations of compact groups. These groups play the role of symmetry- or global gauge groups of the underlying quantum field theory. When they are Lie groups their Lie algebras have the physical interpretation of algebras of conserved charges associated with the superselection rules. Doplicher and Roberts also show how one can reconstruct algebras of charged fields with Bose-Einstein- or Fermi-Dirac statistics from the net of algebras of local observables and the superselection rules.

Unfortunately, most of their results do not hold, in general, for quantum field theories in space-times of dimension two and three. This can be understood by recalling that particles in space-times of dimension three may obey some quantum statistics other than Bose Einstein- or Fermi-Dirac statistics, so-called braid statistics [21]. It had already been noticed in the early seventies that charged fields of certain quantum field theories in two space-time dimensions describing topological solitons can form algebras not described by local commutation- or anticommutation relations; (one then speaks of "exchange algebras" and "fields with braid (group) statistics"). The same phenomena are encountered in three-dimensional Chern-Simons gauge theories. The structure of superselection sectors in such theories cannot be reconstructed from the representation theory of a compact symmetry group, and one must search for an adequate notion of "quantized symmetries", such as quantum groups, whose representation theory reproduces that structure (see, e.g., [10]). It has turned out that the algebraic formulation of quantum field theory provides the right tools leading to a general understanding of braid statistics in two [22] and three [23, 24, 25] space-time dimensions. In these studies, conformal- or gauge invariance were not used. As a consequence, certain structural properties that one can derive e.g., from conformal invariance (structure of local algebras of observables, Haag duality, ...) simply had to be assumed from the outset. The purpose of this paper is to present a general definition of two-dimensional conformal field theories in the context of algebraic quantum field theory and to study their properties by algebraic methods. There are several reasons which motivate this investigation. Before presenting them, we wish to sketch how local algebras arise in conformal field theories on the example of a theory whose chiral algebra is generated by its energy-momentum tensor (i.e., the Virasoro algebra). Some other examples arising from different chiral algebras (current algebras) will be examined in detail in Chap. III.

We assume the central charge $c$ of the theory to be fixed. Because of conformal invariance, the stress-energy tensor splits into a left-moving and a right-moving part, each one defined over a compactified light ray, $S^{1}=\{z \in \mathbb{C}|| z \mid=1\}$, [26]. The left-moving part of the energy-momentum tensor, $T(z)$, for example, can be used to 
define von Neumann algebras of operators acting on the vacuum sector of the theory (i.e., in the representation corresponding to the lowest weight $h=0$ ) by setting

$$
\mathscr{B}(I):=\left\{\exp i T(f) \mid f \text { a real } C^{\infty} \text { function with supp } f \subset I\right\}^{\prime \prime} .
$$

We recall that $\mathscr{C}^{\prime}$ denotes the algebra of bounded operators commuting with $\mathscr{A}$, and $\mathscr{C}^{\prime \prime}:=\left(\mathscr{A}^{\prime}\right)^{\prime}$. In this way we obtain a collection, $\{A(I)\}_{I \subset S^{1}}$, of von Neumann algebras indexed by open, non-dense intervals on $S^{1}$ satisfying the following properties (see Definition 2.3).

(i) Isotony: $I \subseteq J \Rightarrow \mathscr{A}(I) \subseteq \mathscr{A}(J)$.

(ii) Locality: $I \cap J=\varnothing \Rightarrow \mathscr{A}(I) \subseteq \mathscr{A}(J)^{\prime}$.

(iii) Moebius covariance: There exists a unitary representation $\pi_{0}$ of the Moebius group $P S U(1 ; 1)$ on the vacuum sector such that

$$
\pi_{0}(A) \mathscr{b}(I) \pi_{0}(A)^{*}=\mathscr{b}(A \cdot I), \forall A \in P S U(1 ; 1), \forall I \subset S^{1},
$$

(see Appendix I for the notation).

It turns out that, quite independently of the choice of an algebra of local, chiral fields, i.e., of the so-called chiral algebra (e.g. a current algebra), the local algebras (defined similarly as in Eq. (1.1)) are always isomorphic to the unique hyperfinite type $\mathrm{III}_{1}$ factor and satisfy properties (i)-(iii). Of course, the superselection structure of the theory under consideration depends on the choice of the underlying chiral algebra. After passing from chiral algebras of unbounded fields to local von Neumann algebras of bounded operators which, as just mentioned, are universal, the properties of the theory are coded into the assignment of an algebra $\mathscr{A}(I)$ to every interval $I \subset S^{1}$, in such a way that properties (i)-(iii) hold. A more concise way of specifying a conformal field theory is to choose a unitary representation $\pi_{0}$ of the Moebius group $P S U(1 ; 1)$ on a separable Hilbert space $\mathscr{H}$ which "acts properly" on a subalgebra $\mathscr{A}$ contained in the algebra of all bounded operators on $\mathscr{H}$ isomorphic to the hyperfinite type $\mathrm{III}_{1}$ factor. What we mean by a "proper action" of $P S U(1 ; 1)$ on $\mathscr{f}$ will be explained in Definition 2.2.

Next, we explain why we wish to analyse conformal field theories in such generality. One reason originates in an aspect of string theories which we regard as unsatisfactory: their very formulation is usually based on choosing a specific model of classical space-time. This is rather unsatisfactory, because one would hope that a quantum theory of gravity can be formulated without reference to a classical spacetime concept and that it will in fact predict possible models of classical space-time, rather than involve them in its formulation. Following this line of thought, one would like to define the concept of a string vacuum independently of a classical model of space-time and then derive constraints on the structure of space-time from properties of string vacua. It is expected that string vacua can be constructed from $N=2$ superconformal field theories of adequate central charge, and there is growing evidence that one can associate classical space-times (e.g. Calabi-Yau manifolds) to them [27, 28]. We thus consider the model-independent formulation of conformal field theory developed in this work as part of a program aiming at defining what is meant by a string vacuum independently of any a priori ideas about classical space-time and then reconstructing as much as possible of the structure of space-time from algebraic invariants associated with a given string vacuum. In this regard, we expect that a formulation of world-sheet supersymmetry in the framework of algebraic quantum field theory will be of importance for further developments; see the remarks at the end of Chap. II. 
Another one of our motivations comes from the theory of loop groups. Our results show that methods of algebraic quantum field theory are useful to study positive-energy representations of loop groups. In particular, we show how one can introduce a notion of generalized tensor product of representations corresponding to the composition of sectors in algebraic quantum field theory. Similar considerations are also contained in interesting recent work of Wassermann [29].

Finally, as already advocated by Buchholz, Mack and Todorov, algebraic quantum field theory might provide a useful framework for the classification of rational conformal field theories [30]. In particular, we expect that their results concerning the $U(1)$-current algebra can be extended to models based on general loop groups (see Chap. III).

Besides the work of Wassermann and of Buchholz, Mack and Todorov mentioned above, we attract the reader's attention to the work of Buchholz and Schulz-Mirbach [31], of Fredenhagen [32] and to a recent preprint by Guido and Longo which, among other things, contains some very useful results on charge conjugation and Moebius covariance [33]; (similar issues will be discussed in detail in Chap. IV ${ }^{1}$ ). However, as far as we know, there hasn't been, yet, a serious effort aimed at a general definition of conformal and superconformal field theories in the algebraic framework and at investigating detailed structural properties of such theories.

Next, we briefly summarize the contents of this paper. Chapter II is devoted to the definition of conformal field theories in the algebraic context and to the study of properties of the vacuum sector. We emphasize the point of view that a conformal field theory can be defined by specifying a single von Neumann algebra and a unitary representation of the Moebius group acting "properly" on this algebra (Definition 2.2). A complementary point of view is to specify a net of local algebras on a Hilbert space and a vector cyclic and separating for each algebra. It is likely that these two approaches are equivalent. The relation between them is explained in Conjecture 2.27. In Chap. II we also investigate properties of the vacuum sector: the local algebras are shown to be hyperfinite type III 1 factors (Theorem 2.13) if the infinitesimal generator of rotations has certain trace-class properties (Lemma 2.12), and Haag duality is proven to be generally valid (Theorem 2.19). A converse of a recent result of Borchers concerning a relation between the representation of the Moebius group and TomitaTakesaki theory is then proven (Theorem 2.20). We complete our description of the vacuum sector by identifying its local internal symmetries (Lemma 2.22) and by explicitly constructing the antiunitary operators implementing the inversions in the representation of the Moebius group (Theorem 2.26). Some of the technical proofs are given in Appendix II, whereas Appendix I summarizes basic geometrical facts about Moebius transformations and sets up the notation.

In Chap. III, we apply the general results of Chap. II to loop groups of the A-D-E series. Some basic facts about the representation theory of loop groups and of corresponding Kac-Moody algebras are summarized in Sect. III.1-III.7. In Sect. III.8, the vacuum sectors are identified, and, in Sect. III.9, we prove that the local algebras satisfy all the properties of Chap. II in a vacuum representation (Theorem 3.2) and are hyperfinite type $\mathrm{III}_{1}$ factors in charged sectors (Theorem 3.3). The corresponding result for vacuum representations of the Virasoro algebra is stated in Theorem 3.4.

Chapter IV contains an analysis of the superselection structure of conformal field theories. After some definitions and basic properties (Sect. IV.1), we define

\footnotetext{
1 As this work was already written, we received a preprint of Fredenhagen, Rehren and Schroer as well as one by Wiesbrock on related matters; see ref. [77]
} 
a composition of representations in Sect. IV.2. We then analyse subnets associated to the punctured circle and prove that they satisfy essential duality. In Sect. IV.4 we recall the definition of braid statistics operators by now familiar in algebraic field theory [22-25], and in Sect. IV.5, we extend classical results of Doplicher Haag and Roberts $[13,14]$ to the present setting. When analysing representations which are not locally irreducible (see Def. 4.22) we use a technical assumption which is given in Eq. (4.19). This section closes with a conjecture concerning the statistical dimension of a charged sector, Eq. (4.27), which has far reaching consequences. In Sect. IV.6, we sketch some applications to the representation theory of loop groups.

It is worth mentioning that many interesting problems are left unsolved in this work. Besides our conjecture on the statistical dimension of a sector and a careful elaboration of results which are only sketched in Sect. IV.6, we expect that one can construct charged fields ("vertex operators") for chiral WZW-models along the lines presented in [30].

\section{Structure of the Vacuum Representation of a Conformal Field Theory}

\section{II.1. Conformal Nets of Operator Algebras}

Let $\mathscr{H}$ be a separable Hilbert space, $\mathscr{B}(\mathscr{H})$ the set of bounded operators on $\mathscr{H}$, $\mathscr{U}(\mathscr{H})$ its unitary group and $\pi: P S U(1 ; 1) \rightarrow \mathscr{C}(\mathscr{H})$ a strongly continuous, projective representation of the Moebius group. ${ }^{2}$

Remark 2.1. A projective (unitary) representation $\pi$ of $P S U(1 ; 1)$ always defines a projective (unitary) representation of $S U(1 ; 1)$ such that $+\mathbb{1}$ and $-\mathbb{1}$ are mapped onto the same element in $\mathscr{U}(\mathscr{H})$. We find it convenient to denote this representation by the same symbol $\pi$.

Definition 2.2. A representation $\pi$ of the Moebius group on $\mathscr{H}$ acts properly on a von Neumann algebra $\mathscr{A}$ (or equivalently, the von Neumann algebra $\mathscr{A}$ transforms properly under the representation $\pi$ ), if

(i) the one-parameter subgroup of dilatations $D(t)$ is an automorphism group of $\mathscr{b}$ :

$$
\pi(D(t)) \mathscr{C} \pi(D(t))^{*}=\mathscr{A} \quad \forall t \in \mathbb{R},
$$

(ii) the rotation through an angle $\pi$ on $S^{1}$,

$$
z \mapsto-z=R_{0} \cdot z, \quad R_{0}:=\left(\begin{array}{cc}
i & 0 \\
0 & -i
\end{array}\right) \in S U(1 ; 1),
$$

maps $\mathscr{A}$ into its commutant:

$$
\pi\left(R_{0}\right) \mathscr{b} \pi\left(R_{0}\right)^{*} \subseteq \mathscr{C}^{\prime}
$$

(iii) the one-parameter subgroups of translations, $T(p)$ and of special conformal transformations $S(n)$ act as one-sided compressions of $A$ :

$$
\begin{array}{ll}
\pi(T(p)) \mathscr{A} \pi(T(p))^{*} \subseteq \mathscr{A} \quad & \forall p>0, \\
\pi(S(n)) \mathscr{A} \pi(S(n))^{*} \subseteq \mathscr{A} \quad & \forall n>0,
\end{array}
$$

\footnotetext{
2 All group representations considered in this paper are tacitly assumed to be strongly continuous

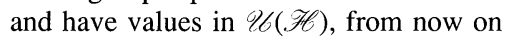




\section{(cf. Appendix I for the notation).}

Our first goal is to show that a von Neumann algebra . $\mathscr{A}$ which transforms properly under the Moebius group determines a local, isotone net of von Neumann algebras which transforms covariantly under the Moebius group.

Definition 2.3. Let $\{\mathscr{t}(I)\}_{I \subset S^{1}}$ be a collection of von Neumann algebras acting on the Hilbert space $\mathscr{H}$, indexed by open, non-dense intervals of the circle. ${ }^{3}$ The collection $\{\mathscr{A}(I)\}_{I \subset S^{1}}$ is called a local, isotone, Moebius covariant net of von Neumann algebras (shortly, a conformal net) if it satisfies the following properties:

(i) $I_{1} \subseteq I_{2} \Rightarrow \mathscr{C}\left(I_{1}\right) \subseteq \mathscr{A}\left(I_{2}\right)$ (isotony);

(ii) $I_{1} \subseteq I_{2}^{\prime} \Rightarrow \mathscr{C}\left(I_{1}\right) \subseteq \mathscr{A}\left(I_{2}\right)^{\prime}$ (locality); here $I^{\prime}:=S^{1} \backslash \bar{I}$;

(iii) there exists a projective representation $\pi$ of the Moebius group on $\mathscr{H}$ such that

$$
\pi(A) \cdot \mathscr{C}(I) \pi(A)^{*}=\mathscr{\ell}(A \cdot I), \forall A \in P S U(1 ; 1), I \subset S^{1},
$$

where $A \cdot I$ denotes the image of the open non-dense interval $I$ under the action of $A$.

Lemma 2.4. (i) If to is a von Neumann algebra transforming properly under a projective representation $\pi$ of the Moebius group on $\mathscr{H}$, then there exists a conformal net $\{. \mathscr{A}(I)\}_{I \subset S^{1}}$ such that $\mathscr{A}=. \mathscr{A}\left(S_{+}\right)$, where $S_{+}:=\left\{z \in S^{1} \mid \operatorname{Im} z>0\right\}$.

(ii) If $\left\{A_{C}(I)\right\}_{I \subset S^{1}}$ is a conformal net of von Neumann algebras acting on a Hilbert space $\mathscr{H}$, then $\mathscr{t}:=. \mathscr{C}\left(S_{+}\right)$transforms properly under the Moebius group.

Proof. For the proof it will be convenient to use the non-compact picture of $S^{1}$ obtained by performing a stereographic projection which maps $-1 \in S^{1}$ onto $\infty$ (see Appendix I for details). Under this map, $S U(1 ; 1)$ is mapped onto $S L(2 ; \mathbb{R})$ and the action of $S U(1 ; 1)$ on $S^{1}$ corresponds to the action of $S L(2 ; \mathbb{R})$ as fractional linear transformations on $\mathbb{R}$.

Ad (i). If . th transforms properly under $S U(1 ; 1)$, we define

$$
\begin{aligned}
& \mathscr{b}\left(S_{+}\right):=\mathscr{b}, \\
& \mathscr{A}(I):=\pi(A) \cdot \mathscr{b} \pi(A)^{*},
\end{aligned}
$$

where $A \in S U(1 ; 1)$ is a group element which maps $S_{+}$onto $I: A \cdot S_{+}=I$. We first have to show that $\mathscr{A}(I)$ is well defined. Let $A_{1}, A_{2}$ be two elements of $S U(1 ; 1)$ which satisfy $A_{i} \cdot S_{+}=I, i=1,2$. Then $A_{3}:=A_{1}^{-1} A_{2}$ maps $S_{+}$onto $S_{+}$and the endpoint set $\{+1 ;-1\}$ of $S_{+}$onto itself. There are two possibilities:

(a) $A_{3}(+1)=+1$ and $A_{3}(-1)=-1$. It is then easy to check that $A_{3}= \pm D(t)$, for some $t$.

(b) $A_{3}(+1)=-1$ and $A_{3}(-1)=+1$. It follows that $A_{3}= \pm\left(\begin{array}{cc}i & 0 \\ 0 & -i\end{array}\right) D(t)$ for
some $t$, so that $A_{3}$ maps $S_{+}$onto $S_{-}$.

Therefore only case (a) can occur. By assumption, $\pi( \pm D(t))$ is an automorphism of . $\ell\left(S_{+}\right)$so that the right-hand side of Eq. (2.2) is independent of the choice of $A$.

To prove locality, it is sufficient to check that $\mathscr{A}\left(S_{-}\right) \subseteq \mathscr{A}\left(S_{+}\right)^{\prime}$. But

$$
\mathscr{A}\left(S_{-}\right)=\pi\left(R_{0}\right) \mathscr{A}\left(S_{+}\right) \pi\left(R_{0}\right)^{*} \subseteq \mathscr{C}^{\prime}=\mathscr{A}\left(S_{+}\right)^{\prime}
$$

by assumption.

\footnotetext{
3 Throughout this work, the intervals $I \subset S^{1}$ considered are always non-dense and open
} 
To prove isotony, it is sufficient to check that $\mathscr{A}(I) \subseteq \mathscr{A}\left(S_{+}\right)$for $I \subseteq S_{+}$. This is clearly the case if $I=T(a) \cdot S_{+}$or $I=S(a) \cdot S_{+}$, for a positive $a$. Let $I$ correspond to the interval $\left(c_{0} ; c_{1}\right) \subseteq \mathbb{R}_{+}, c_{0}, c_{1} \in \mathbb{R}_{+}$in the real picture. Then

$$
\tilde{S}\left(\frac{1}{c_{1}}\right) \tilde{T}\left(\frac{c_{0} c_{1}}{c_{1}-c_{0}}\right) \cdot \mathbb{R}_{+}=\left(c_{0} ; c_{1}\right) .
$$

Hence, $I=S\left(\frac{1}{c_{1}}\right) T\left(\frac{c_{0} c_{1}}{c_{1}-c_{0}}\right) \cdot S_{+}, 0<c_{0}<c_{1}<\infty$ and by assumption, $\mathscr{b}(I) \subseteq \mathscr{A}\left(S_{+}\right)$. Covariance of the net is obvious.

Ad (ii). In the real picture, $\mathbb{R}_{+}$is the image of $S_{+}$. Since

$$
\begin{aligned}
& \tilde{D}(t) \cdot \mathbb{R}_{+}=\mathbb{R}_{+}, \quad \forall t \in \mathbb{R}, \\
& \tilde{T}(a) \cdot \mathbb{R}_{+}=(a ; \infty) \subseteq \mathbb{R}_{+}, \quad a>0, \\
& \tilde{S}(a) \cdot \mathbb{R}_{+}=\left(0 ; \frac{1}{a}\right) \subseteq \mathbb{R}_{+}, \quad a>0,
\end{aligned}
$$

(i) and (iii) of Definition 2.2 follow by covariance of the net and isotony.

Finally, by covariance and locality,

$$
\pi\left(R_{0}\right) \mathscr{A}\left(S_{+}\right) \pi\left(R_{0}\right)^{*}=\mathscr{b}\left(R_{0} \cdot S_{+}\right)=\mathscr{A}\left(S_{-}\right) \subseteq A\left(S_{+}\right)^{\prime},
$$

so that (ii) of Definition 2.2. holds. This completes the proof of the lemma.

We now restrict our attention to an algebra $\mathscr{C}$ and a representation $\pi_{0}$ of the Moebius group associated with "the vacuum sector" of a conformal field theory. That is, we assume the existence of a unique "vacuum vector" invariant under the action of $P S U(1 ; 1)$.

Definition 2.5. Let $\mathscr{H}$ be a separable Hilbert space, $\pi_{0}$ a unitary representation of the Moebius group on $\mathscr{H}$ which acts properly on a von Neumann algebra $\mathscr{A}$. We denote by $\{\mathscr{b}(I)\}_{I \subset S^{1}}$ the conformal net constructed in Lemma 2.3. Let us assume that

(i) the spectrum of the generator of rotations of $P S U(1 ; 1)$ is positive (positiveenergy representation).

(ii) There exists a unique vector $\Omega \in \mathscr{H}$ invariant under $P S U(1 ; 1)$, (vacuum vector).

(iii) $\Omega$ is cyclic for the von Neumann algebra $\mathfrak{A}:=\left\{\bigcup_{I \subset S^{1}} \mathscr{f}(I)\right\}^{\prime \prime}$ generated by
the net $\{\mathscr{C}(I)\}_{I \subset S^{1}}$.

If properties (i)-(iii) are satisfied, we say that $\left\{\mathscr{H} ; \pi_{0} ; \mathscr{b} ; \Omega\right\}$ determines the vacuum sector of a conformal field theory, or, equivalently, the vacuum representation of the conformal net $\{\mathscr{b}(I)\}_{I \subset S^{1}}$.

In a positive-energy representation, the generators of translations and of special conformal transformations have the following spectral properties.

Lemma 2.6. The spectrum of the generator of translations is always positive in a positive-energy representation $\pi_{0}$ of $P S U(1 ; 1)$. The spectrum of the generator of special conformal transformations is negative.

Proof. A unitary representation $\pi_{0}$ of $P S U(1 ; 1)$ lifts to a unitary representation $\pi_{0}$ of $S U(1 ; 1)$ such that $\pi_{0}(-\mathbb{1})=\pi_{0}(\mathbb{1})$. The positive-energy condition means that the spectrum of the generator of rotations $R(t)$ is positive (cf. Appendix I for 
the notation). The only irreducible unitary representations of $S L(2 ; \mathbb{R}) \cong S U(1 ; 1)$ which fulfill these two conditions are members of the holomorphic discrete series [40]: $\pi_{n}^{+}, n \in 2 \cdot \mathbb{Z}_{+}$. Hence $\pi_{0}$ is a direct sum of irreducible representations of the holomorphic discrete series. One checks easily, in the realization of these representations by operators acting on holomorphic functions on the upper half plane [41], that the spectrum of the generator of translations is positive. Similar arguments may be found in [42].

Since the one-parameter subgroups of translations and of special conformal transformations are conjugate in $S L(2 ; \mathbb{R}), R_{0} T(p) R_{0}^{-1}=S(-p)$, it follows that the spectrum of the generator of special conformal transformations is negative. This completes the proof of the lemma.

We now derive some standard properties of the local algebras in the vacuum sector. Most of our arguments are adaptations of well-known results to the present setting. The proof of Haag duality given in Sect. II.2 is an application of a recent theorem of Borchers [34]. The following theorem is obtained by applying twice the Reeh-Schlieder theorem.

Theorem 2.7. Let $\left\{\mathscr{H} ; \pi_{0} ; \mathscr{A} ; \Omega\right\}$ be the vacuum sector of a conformal field theory. Then

$$
\overline{\mathfrak{A} \Omega}=\overline{\mathscr{A} \Omega},
$$

where the symbol means closure in the Hilbert space norm, and $\mathfrak{A}$ has been defined in Definition 2.5, (iii).

Corollary 2.8. (Reeh-Schlieder) The vacuum vector $\Omega$ is cyclic and separating for each local algebra $\mathscr{b}(I), I \subset S^{1}$.

Proof of Corollary 2.8. Since the net $\{\mathscr{b}(I)\}_{I \subset S^{1}}$ is Moebius-covariant and $\Omega$ is invariant under Moebius transformations, it follows at once from Theorem 2.7 that $\Omega$ is cyclic for any local algebra $\mathscr{A}(I)$. The separating property is now a consequence of locality. This completes the proof of the corollary.

Proof of Theorem 2.7. Given an interval $I \subset S^{1}$ define

$$
\mathscr{S}(I):=\left\{\bigcup_{t \in \mathbb{R} / 2 \mathbb{Z}} \pi_{0}(R(t)) \mathscr{C}(I) \pi_{0}(R(t))^{*}\right\}^{\prime \prime} .
$$

If $l(I)$ is the length of the interval $I$, normalized in such a way that $l\left(S^{1}\right)=2 \pi$, then the von Neumann algebras $\mathscr{B}(I)$ have the following properties:

(i) $l(I)=l(J)$ implies $\mathscr{B}(I)=\mathscr{B}(J)$.

(ii) $l(I)<l(J)$ implies $\mathscr{B}(I) \subseteq \mathscr{B}(J)$.

(iii) If $\left\{I_{n}\right\}_{n=1,2, \ldots}$ is an increasing sequence of intervals such that $l\left(I_{n}\right) \rightarrow 2 \pi$ $(n \rightarrow \infty)$ then

$$
\mathfrak{A}=\left(\bigcup_{n} \mathscr{B}\left(I_{n}\right)\right)^{\prime \prime}
$$

(iv) The one-parameter group of rotations $\pi_{0}(R(t))$ induces automorphisms of $\mathscr{B}(I)$ satisfying the spectrum condition. By applying the Reeh-Schlieder theorem, as given in [35], we obtain that

$$
\overline{\mathscr{B}(I) \Omega} \subseteq \overline{\mathscr{A}(J) \Omega},
$$

where $J$ is any interval on $S^{1}$ such that $l(J)>l(I)$. 
Next, we consider intervals on the circle of the type $I=(\alpha ;-1), \alpha \in S^{1} \backslash\{-1\}$ (see Appendix I for the notation, or the next figure),

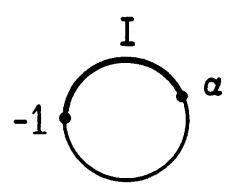

and define

$$
\mathscr{C}:=\left\{\bigcup_{\substack{I=(\alpha ;-1) \\ \alpha \in S^{1} \backslash\{-1\}}} \mathscr{A}(I)\right\}^{\prime \prime} .
$$

The von Neumann algebra $\mathscr{C}$ has the following properties:

(v) The translations define a one-parameter automorphism group of $\mathscr{C}$ satisfying the spectrum condition.

(vi) If $\left\{J_{n}=\left(\alpha_{n} ;-1\right), \alpha_{n} \in S^{1} \backslash\{-1\}\right\}_{n=1,2, \ldots}$ is an increasing sequence of intervals such that $l\left(J_{n}\right) \rightarrow 2 \pi(n \rightarrow \infty)$ then $\mathscr{A}\left(J_{n}\right) \subseteq \mathscr{A}\left(J_{n+1}\right)$ and

$$
\mathscr{C}=\left(\bigcup_{n} \mathscr{C}\left(J_{n}\right)\right)^{\prime \prime} \text {. }
$$

(vii) Applying once again the Reeh-Schlieder theorem [35] we see that

$$
\overline{\mathscr{C} \Omega} \subseteq \overline{\mathscr{A}\left(S_{+}\right) \Omega} \text {, with } \mathscr{f}\left(S_{+}\right) \equiv \mathscr{A} \text {. }
$$

Let $\left\{I_{n}\right\}_{n=1,2, \ldots}$ be an increasing sequence of intervals as in (iii). Then

$$
\overline{\mathfrak{A} \Omega}=\overline{\left(\bigcup_{n} \mathscr{B}\left(I_{n}\right)\right)^{-s} \Omega},
$$

where $^{-s}$ means strong closure. But

$$
\begin{aligned}
\overline{\left(\bigcup_{n} \mathscr{B}\left(I_{n}\right)\right)^{-s} \Omega} & =\overline{\left(\bigcup_{n} \mathscr{B}\left(I_{n}\right)\right) \Omega} \\
& =\overline{\bigcup_{n}\left(\mathscr{B}\left(I_{n}\right) \Omega\right)} \\
& \subseteq \overline{\bigcup_{n} \overline{\mathscr{B}\left(I_{n}\right) \Omega}} \subseteq \overline{\bigcup_{n} \overline{\mathscr{B}\left(J_{n}\right) \Omega}} .
\end{aligned}
$$

Here we applied the Reeh-Schlieder theorem as formulated in (iv) and we chose $J_{n}$ such that $J_{n}=\left(\alpha_{n} ;-1\right), l\left(J_{n}\right)>l\left(I_{n}\right)$, i.e., the sequence of intervals $\left\{J_{n}\right\}_{n=1,2, \ldots}$ satisfies the properties of (vi). Hence,

$$
\begin{aligned}
\overline{\bigcup_{n} \overline{\mathscr{A}\left(J_{n}\right) \Omega}} & =\overline{\bigcup_{n}\left(\mathscr{C}\left(J_{n}\right) \Omega\right)}=\overline{\left(\bigcup_{n} \mathscr{A}\left(J_{n}\right)\right) \Omega} \\
& =\overline{\mathscr{C} \Omega} \subseteq \overline{\mathscr{A}\left(S_{+}\right) \Omega}
\end{aligned}
$$


where we applied again the Reeh-Schlieder theorem, as given in (vii). We have shown that

$$
\overline{\mathfrak{A} \Omega} \subseteq \overline{\mathscr{A}\left(S_{+}\right) \Omega} .
$$

Since the converse inclusion is obvious, this concludes the proof of the theorem.

The Reeh-Schlieder property means that each local algebra $A(I), I \subset S^{1}$ is in standard form [36]. We can then define the Tomita operators [36] $S_{I}, \forall I \subset S^{1}$, as the closure of

$$
S_{I} A \Omega:=A^{*} \Omega, \quad A \in \mathcal{A}(I) .
$$

The modular conjugation $J_{I}$ and the modular operator $\Delta_{I}^{1 / 2}$ are obtained by polar decomposition of $S_{I}$,

$$
S_{I}=J_{I} \cdot \Delta_{I}^{1 / 2}
$$

They have the property [36] that

$$
\begin{aligned}
J_{I} \mathscr{C}(I) J_{I} & =\mathscr{A}(I)^{\prime}, \\
\Delta_{I}^{i t} \mathscr{A}(I) \Delta_{I}^{-i t} & =\mathscr{A}(I), \quad \forall I \subset S^{1}, t \in \mathbb{R} .
\end{aligned}
$$

We will show, later on, that these operators always implement geometric transformations [37] in the present situation.

We may now use well-known arguments of Driessler [43] to show that the local algebras of the vacuum sector of a conformal field theory are factors of type III $_{1}$ [44].

Lemma 2.9. In the vacuum sector of a conformal field theory, the local algebras . $A(I), I \subset S^{1}$, are factors of type $I I I_{1}$.

Proof. It is sufficient to show that $\mathscr{t}=\mathscr{C}\left(S_{+}\right)$is a factor of type $\mathrm{III}_{1}$. In the real picture, $S_{+}$is mapped onto $\mathbb{R}_{+}$. Since $\tilde{T}(a) \mathbb{R}_{+} \subseteq \mathbb{R}_{+}, \forall a>0$, it follows that $\mathbb{R}_{+}$is monotone in the sense of [43]. Let $P_{\Omega}$ be the orthogonal projection onto the vacuum vector $\Omega$. It follows from the spectral properties of the translation operator in a positive-energy representation $\pi_{0}$ of $P S U(1 ; 1)$ that $\pi_{0}(T(a))$ converges weakly to $P_{\Omega}$ as $a \rightarrow \infty$ [45]. The proof of the lemma is now completed by applying Theorem 2.2. of [43].

Definition 2.10. Let $I_{1}, I_{2}$ be two intervals on the circle; we write $I_{1} \Subset I_{2}$ if the closure of $I_{1}$ is contained in the open interval $I_{2}$.

Definition 2.11. The split property is said to hold for a conformal net if, given two intervals $I_{1}, I_{2}$ such that $I_{1} \Subset I_{2}$, there exists a type $I_{\infty}$ factor $\mathscr{M}$ such that

$$
\mathscr{A}\left(I_{1}\right) \subseteq \mathscr{M} \subseteq \subseteq \mathscr{A}\left(I_{2}\right)
$$

The split property for a vacuum conformal net follows from a result of Buchholz, D'Antoni and Fredenhagen [46]. We formulate this result under assumptions which are typical of conformal field theories constructed from the representation theory of loop groups (see [47], Remark 13.13).

Lemma 2.12. Let $\left\{\mathscr{H} ; \pi_{0} ; \mathscr{A} ; \Omega\right\}$ be the vacuum sector of a conformal field theory and $K$ the generator of the one-parameter group of rotations on $S^{1}$. If for $\beta>0$ the operator $e^{-\beta K}$ is trace-class and there exist positive constants $\beta_{0}$, n such that

$$
\left\|e^{-\beta K}\right\|_{1} \leq e^{\left(\beta_{0} / \beta\right)^{n}}, \quad \beta>0, \beta \rightarrow 0
$$

holds then the split property holds for the net $\{. A(I)\}_{I \subset S^{1}}$ of Definition 2.5. 
Proof. The results of [46] are formulated in terms of a local net on Minkowski space-time. However, the proof given there does not use any properties specific to this particular situation. We reformulate the theorem of Buchholz, D'Antoni and Fredenhagen under prerequisites which are sufficiently general for our purposes: ${ }^{4}$

(i) Let $\mathscr{A}_{1} \subseteq \mathscr{A}_{2} \subseteq \mathscr{A}_{3} \subseteq \mathscr{A}_{4}$ be a sequence of inclusions of von Neumann algebras.

(ii) Let $U(t)$ be a one-parameter group of unitaries such that for $|t|<\delta$,

$$
U(t) \mathscr{A}_{i} U(t)^{*} \subseteq \mathscr{A}_{i+1}, \quad i=1,2,3 .
$$

(iii) Assume that the generator $K$ of $U(t)=e^{i K t}$ has positive spectrum including the eigenvalue zero with multiplicity one, and let $\Omega$ be the unique (up to a phase) corresponding unit eigenvector; $\Omega$ is supposed to be cyclic and separating for $\mathscr{A}_{i}$, $i=1,2,3,4$.

(iv) Assume that the maps $\theta_{\beta}: \mathscr{b}_{\imath} \rightarrow \mathscr{H}, i=1,2,3,4, \beta>0$ defined by

$$
\theta_{\beta}(A):=e^{-\beta K} A \Omega, \quad A \in \mathscr{C}_{i}, i=1,2,3,4
$$

are nuclear and that the estimate (2.5) holds, as $\beta$ tends to zero, for the trace norm of $\theta_{\beta}$ (see [46]). Then there exists a type $I_{\infty}$ factor $\mathscr{M} b$ such that $\mathscr{A}_{1} \subseteq \mathscr{A b} \subseteq \mathscr{b}_{4}$.

The proof follows word for word the one given in [46] with the identification $\mathscr{b}_{1} \rightarrow \mathscr{b}\left(\mathscr{O}_{a}\right), \mathscr{b}_{4} \rightarrow \mathscr{A}\left(\mathscr{O}_{b}^{\prime}\right), \mathscr{b}_{2} \rightarrow \mathscr{A}\left(\tilde{\mathscr{O}}_{a}\right), \mathscr{A}_{3} \rightarrow \mathscr{A}\left(\tilde{\mathscr{O}}_{b}\right)$.

Under the assumptions of the lemma, $e^{-\beta K}$ is trace class so that the maps $\theta_{\beta}$ are nuclear for any local algebra $\mathscr{\ell}(I), I \subset S^{1}$. Given $I_{1} \Subset I_{4}$, there exist $I_{2}, I_{3}$, such that $I_{1} \Subset I_{2} \Subset I_{3} \Subset I_{4}$. Setting $\mathscr{C}_{i}:=\mathscr{C}\left(I_{i}\right), i=1,2,3,4$, and applying the previous argument completes the proof of the lemma.

An immediate consequence [50] of Lemma 2.12 is that the local algebras in the vacuum sector of a conformal field theory are hyperfinite (i.e. are the weak limit of matrix algebras), since $\mathscr{A}(I), I \subset S^{1}$, can be continuously approximated from the inside (or the outside) by local algebras [31]. We summarize the previous discussion in the following theorem.

Theorem 2.13. Under the assumptions introduced in Definition 2.5 and in Lemma 2.12, each local algebra $\mathscr{f}(I), I \subset S^{1}$ in the vacuum sector of a conformal field theory is isomorphic to the unique hyperfinite factor of type $I I I_{1}$.

Remark 2.14. It follows from Theorem 2.13 that the whole information about a conformal quantum field theory is encoded in the map

$$
I \rightarrow \mathscr{A}(I)
$$

assigning to each interval a hyperfinite type $I I I_{1}$ factor in $\mathscr{H}$. As observed in Lemma 2.4, the map (2.6) is fully determined by the representation $\pi_{0}$ of the Moebius group on the vacuum sector and the algebra $\mathscr{b}=\mathscr{A}\left(S_{+}\right)$. We shall also prove, in Theorem 2.19, that the net $I \mapsto \mathscr{C}(I)$ and the vacuum state $\Omega$ already determine the representation $\pi_{0}$ of the Moebius group (provided $\pi_{0}$ exists).

$\overline{4}$ The following conditions are certainly not optimal. For recent developments, see $[48,49]$ 


\section{II.2 Moebius Covariance and Tomita-Takesaki Modular Operators; Haag Duality}

We start this section with a definition of Haag duality.

Definition 2.15. In the vacuum sector of a conformal field theory, the net $\{. \mathscr{A}(I)\}_{I \subset S^{1}}$ is said to satisfy Haag duality (to be dual), if for any open, non-dense interval $I \subset S^{1}$,

$$
\mathscr{b}(I)^{\prime}=\mathscr{A}\left(I^{\prime}\right)
$$

holds, where $I^{\prime}:=\left(S^{1} \backslash I\right)^{0}$ is the interior of the complement of $I$ in $S^{1}$.

Haag duality was checked by different methods in several models [51-53]. It is a crucial property for the applicability of the algebraic framework to the analysis of the superselection structure of quantum field theories [13]. It is then natural to investigate under which general assumptions models give rise to local algebras satisfying Haag duality. J. Bisognano and E. Wichmann showed [37] that Wightman quantum field theories lead to (essentially) dual nets, provided that the underlying quantum fields satisfy some regularity conditions (see also [54] for a general formulation of the Bisognano-Wichmann theorem). Recently, D. Buchholz and H. Schulz-Mirbach [31] adapted the arguments of J. Bisognano and E. Wichmann to prove Haag duality for conformal field theories. We shall give a proof of duality for the net $\{\mathscr{f}(I)\}_{I \subset S^{1}}$ in the vacuum sector of a conformal field theory which does not make reference to the underlying (Wightman) quantum fields. This result will then be applied to the construction of models, starting from the representation theory of loop groups.

Let $S U(1 ; 1)_{ \pm}$be the extension of $S U(1 ; 1)$ by matrices having determinant -1 and $P S U(1 ; 1)_{ \pm}$the corresponding group of geometric transformations of the circle (see Appendix I). We denote by $\mathscr{C}(\mathscr{H} \mathscr{b})_{\text {_ }}$ the set of antilinear unitary operators in $\mathscr{H}$ and by $\mathscr{C}(\mathscr{H})_{ \pm}$the group of unitary and antiunitary operators on $\mathscr{H}$. Following the terminology introduced by Wigner [55], we define a corepresentation as follows.

Definition 2.16. Let $G$ be a group. A homomorphism

$$
\hat{\pi}: G \rightarrow \mathscr{C}(\mathscr{H})_{ \pm}
$$

into the group of (anti-) unitary operators of a Hilbert space $\mathscr{H}$ is, called a corepresentation of $G$.

Remark 2.17. (i) Clearly, a group $G$ admits non-trivial corepresentations $\hat{\pi}$ only if it has a normal subgroup $G_{0}$ of order two such that $\hat{\pi}\left(G_{0}\right) \subseteq \mathscr{U} \mathscr{C}(\mathscr{H})$. We will denote by $\pi$ the restriction of $\hat{\pi}$ to $G_{0}$.

(ii) We are interested in strongly continuous corepresentations of $S U(1 ; 1)_{ \pm}$which map matrices of determinant $+1,-1$ into $\mathscr{C}(\mathscr{H})_{+}, \mathscr{U}(\mathscr{H})_{-}$, respectively.

(iii) Let $\hat{\pi}$ be a corepresentation of $S U(1 ; 1)_{ \pm}$. Then $\pi:=\left.\hat{\pi}\right|_{S U(1 ; 1)}$ is a unitary representation of $S U(1 ; 1)$, and $\tilde{J}_{S_{+}}:=\hat{\pi}\left(I_{S_{+}}\right)$is a conjugation $\left(\tilde{J}_{S_{+}}^{2}=\mathbb{1}\right)$ satisfying

$$
\tilde{J}_{S_{+}} \pi(A) \tilde{J}_{S_{+}}=\pi(\bar{A}), \quad \forall A \in S U(1 ; 1) .
$$

Conversely, since $S U(1 ; 1)_{ \pm}$is the semi-direct product of $S U(1 ; 1)$ and $\left\{\mathbb{1} ; I_{S_{+}}\right\}$ by the outer automorphism $\phi$ of $S U(1 ; 1)$ which maps $A \in S U(1 ; 1)$ onto $\bar{A}$ (see Appendix I), given a representation $\pi$ of $S U(1 ; 1)$ and an antiunitary conjugation $\tilde{J}_{S_{+}}$such that Eq. (2.7) holds, we can construct a corepresentation $\hat{\pi}$ of $S U(1 ; 1)_{ \pm}$ such that $\left.\hat{\pi}\right|_{S U(1 ; 1)}=\pi$ and $\hat{\pi}\left(I_{S_{+}}\right)=\tilde{J}_{S_{+}}$. 
Definition 2.18. Let $\hat{\pi}$ be a corepresentation of $P S U(1 ; 1)_{ \pm}$on the Hilbert space $\mathscr{H}$. A local net $\{\mathscr{b}(I)\}_{I \subset S^{1}}$ is said to transform covariantly, under improper Moebius transformations if

$$
\hat{\pi}(A) \mathscr{A}(I) \hat{\pi}(A)^{*}=\mathscr{A}(A \cdot I), \quad \forall A \in P S U(1 ; 1)_{ \pm}, I \subset S^{1},
$$

where $A \cdot I$ denotes the image of $I$ under the action of $A$.

Theorem 2.19. Let $\left\{\mathscr{H} ; \pi_{0} ; \mathscr{b} ; \Omega\right\}$ be the vacuum sector of a conformal field theory.

(i) The modular conjugation $J_{S_{+}}$of $\mathscr{b}=\mathscr{A}\left(S_{+}\right)$with respect to $\Omega$ satisfies the following commutation relations with $S U(1 ; 1)$ :

$$
J_{S_{+}} \pi_{0}(A) J_{S_{+}}=\pi_{0}(\bar{A}), \quad \forall A \in S U(1 ; 1) ;
$$

consequently, $\pi_{0}$ extends to a corepresentation $\hat{\pi}_{0}$ of $S U(1 ; 1)_{ \pm}$on $\mathscr{H}$.

(ii) The net $\{\mathscr{f}(I)\}_{I \subset S^{1}}$ is dual and transforms covariantly under the corepresentation $\hat{\pi}_{0}$ of the improper Moebius transformations.

(iii) The representation $\pi_{0}$ of the Moebius group is uniquely determined by the net

$$
I \mapsto \mathscr{C}(I)
$$

and the vacuum vector $\Omega$. It is the unique representation of $S U(1 ; 1)$ on $\mathscr{H}$ for which the generator of translations has positive spectrum, which has $\Omega$ as an invariant vector and under which the net transforms covariantly.

(iv) The one-parameter group of dilatations of $S U(1 ; 1)$ coincides with the modular group of the algebra $\mathscr{A}\left(S_{+}\right)$:

$$
\pi_{0}(D(t))=\Delta_{S_{+}}^{i t} .
$$

Proof. Ad (i). Since any element of $S U(1 ; 1)$ can be written as a product of dilatations, translations and special conformal transformations (see Appendix I), it is sufficient to check that $J_{S_{+}}$has the right commutation relations with these three one-parameter subgroups. That is, the equations

$$
\begin{aligned}
& J_{S_{+}} \pi_{0}(T(p)) J_{S_{+}}=\pi_{0}(T(-p)), \\
& J_{S_{+}} \pi_{0}(S(n)) J_{S_{+}}=\pi_{0}(T(-n)), \\
& J_{S_{+}} \pi_{0}(D(t)) J_{S_{+}}=\pi_{0}(D(t)),
\end{aligned}
$$

hold. By Lemma 2.6 the generator of the one-parameter group $V(p):=\pi_{0}(T(p))$ has positive spectrum and the generator of $W(n):=\pi_{0}(S(n))$ has negative spectrum. By assumption, $V(p), p>0$, and $W(n), n>0$, act as one-sided compressions of the algebra $\mathscr{A}=\mathscr{A}\left(S_{+}\right)$(see Def. 2.2). Hence, we may apply Theorem II.9 of Borchers [34] to obtain

$$
\begin{aligned}
J_{S_{+}} \pi_{0}(T(p)) J_{S_{+}} & =\pi_{0}(T(-p)) \\
\Delta_{S_{+}}^{i t} \pi_{0}(T(p)) \Delta_{S_{+}}^{-t t} & =\pi_{0}\left(T\left(e^{-2 \pi t} p\right)\right) \\
& =\pi_{0}\left(D(t) T(p) D(t)^{-1}\right)
\end{aligned}
$$

and

$$
\begin{aligned}
J_{S_{+}} \pi_{0}(S(n)) J_{S_{+}} & =\pi_{0}(T(-n)) \\
\Delta_{S_{+}}^{i t} \pi_{0}(S(n)) \Delta_{S_{+}}^{-i t} & =\pi_{0}\left(S\left(e^{+2 \pi t} n\right)\right) \\
& =\pi_{0}\left(D(t) S(n) D(t)^{-1}\right)
\end{aligned}
$$


Since $\pi_{0}(D(s))$ is a one-parameter group of automorphisms of teaving the vacuum vector $\Omega$ invariant, it follows from a well-known result of Takesaki [36] that $\pi_{0}(D(s))$ commutes with $J_{S_{+}}$and $\Delta_{S_{+}}^{i t}$ :

$$
\begin{aligned}
J_{S_{+}} \pi_{0}(D(s)) J_{S_{+}} & =\pi_{0}(D(s)), \\
\Delta_{S_{+}}^{i t} \pi_{0}(D(s)) \Delta_{S_{+}}^{-i t} & =\pi_{0}(D(s)) .
\end{aligned}
$$

This completes the proof of (i).

Ad (ii). We first prove duality. It is sufficient to prove the $\mathscr{A}\left(S_{-}\right)=\mathscr{A}\left(S_{+}\right)^{\prime}$ since by $S U(1 ; 1)$-covariance of the net, this implies $\mathscr{C}(I)^{\prime}=\mathscr{C}\left(I^{\prime}\right)$ for an arbitrary interval $I \subset S^{1}$. From locality, we know that

$$
\mathscr{A}\left(S_{-}\right) \subseteq \mathscr{A}\left(S_{+}\right)^{\prime}
$$

so that it is sufficient to check the converse inclusion. The rotation $R_{0}=R(1 / 2)=$ $\left(\begin{array}{rr}i & 0 \\ 0 & -i\end{array}\right) \in S U(1 ; 1)$ maps $S_{+}$onto $S_{-}$and satisfies $\bar{R}_{0}=R_{0}^{-1}$. Hence,

$$
\begin{aligned}
\mathscr{C}\left(S_{-}\right) & =\pi_{0}\left(R_{0}\right) \mathscr{A}\left(S_{+}\right) \pi_{0}\left(R_{0}\right)^{*} \\
& =\pi_{0}\left(R_{0}\right) \mathscr{A}\left(S_{+}\right) \pi_{0}\left(R_{0}^{-1}\right),
\end{aligned}
$$

by covariance of the net under $S U(1 ; 1)$. Thus,

$$
\begin{aligned}
J_{S_{+}} \mathscr{A}\left(S_{-}\right) J_{S_{+}} & =J_{S_{+}} \pi_{0}\left(R_{0}\right) \mathscr{A}\left(S_{+}\right) \pi_{0}\left(R_{0}^{-1}\right) J_{S_{+}} \\
& =\pi_{0}\left(\bar{R}_{0}\right) J_{S_{+}} \mathscr{A}\left(S_{+}\right) J_{S_{+}} \pi_{0}\left(R_{0}\right) \\
& =\pi_{0}\left(R_{0}^{-1}\right) \mathscr{C}\left(S_{+}\right)^{\prime} \pi_{0}\left(R_{0}\right) \\
& =\left(\pi_{0}\left(R_{0}^{-1}\right) \mathscr{A}\left(S_{+}\right) \pi_{0}\left(R_{0}\right)\right)^{\prime} \\
& =\mathscr{A}\left(S_{-}\right)^{\prime},
\end{aligned}
$$

where we used successively the commutation relations shown in (i), property (2.3) of the modular conjugation and the covariance of the net under $S U(1 ; 1)$. Conjugating on both sides by $J_{S_{+}}$we obtain

$$
\mathscr{A}\left(S_{-}\right)=J_{S_{+}} \mathscr{A}\left(S_{-}\right)^{\prime} J_{S_{+}} .
$$

By locality, $\mathscr{A}\left(S_{+}\right) \subseteq \mathscr{A}\left(S_{-}\right)^{\prime}$ so that

$$
\mathscr{C}\left(S_{+}\right)^{\prime}=J_{S_{+}} \mathscr{A}\left(S_{+}\right) J_{S_{+}} \subseteq J_{S_{+}} \mathscr{A}\left(S_{-}\right)^{\prime} J_{S_{+}}=\mathscr{A}\left(S_{-}\right) .
$$

The first equality follows again by Tomita-Takesaki theory and the second from Eq. (2.11). This proves duality.

To prove $S U(1 ; 1)_{ \pm}$covariance of the net, it is sufficient to check that the net transforms covariantly under $J_{S_{+}}$, since $S U(1 ; 1)_{ \pm}$is the semi-direct product of $S U(1 ; 1)$ and $\left\{\mathbb{1} ; I_{S_{+}}\right\}$. Let $I \subset S^{1}$ be an interval and $A \in S U(1 ; 1)$ be such that $A \cdot S_{+}=I$. Then by $S U(1 ; 1)$-covariance of the net, (i) and duality,

$$
\begin{aligned}
J_{S_{+}} \mathscr{A}(I) J_{S_{+}} & =J_{S_{+}} \pi_{0}(A) \mathscr{A}\left(S_{+}\right) \pi_{0}\left(A^{-1}\right) J_{S_{+}} \\
& =\pi_{0}(\bar{A}) J_{S_{+}} \mathscr{A}\left(S_{+}\right) J_{S_{+}} \pi_{0}\left(\bar{A}^{-1}\right) \\
& =\pi_{0}(\bar{A}) \mathscr{A}\left(S_{-}\right) \pi_{0}\left(\bar{A}^{-1}\right) \\
& =\mathscr{A}\left(\bar{A} \cdot S_{-}\right) .
\end{aligned}
$$


But $\bar{A} \cdot S_{-}=\bar{A} \cdot I_{S_{+}} \cdot S_{+}=\bar{A} \cdot I_{S_{+}} \cdot A^{-1} \cdot I$, and one checks by direct computation that $\bar{A} \cdot I_{S_{+}} \cdot A^{-1}=I_{S_{+}}, \forall A \in S U(1 ; 1)$. Hence,

$$
J_{S_{+}} \mathscr{b}(I) J_{S_{+}}=\mathscr{A}\left(I_{S_{+}} \cdot I\right)
$$

This completes the proof of (ii).

Ad (iii). Let $(\alpha ; \beta),(\gamma ; \delta) \subset S^{1}$ and $A \in S U(1 ; 1)$ be such that $A \cdot(\alpha ; \beta)=(\gamma ; \delta)$ (see Appendix I for the notation). If $\left\{J_{(\alpha ; \beta)} ; \Delta_{(\alpha ; \beta)}^{1 / 2}\right\}$ is the modular data associated to $\{\mathscr{f}((\alpha ; \beta)) ; \Omega\}$ (see Eqs. (2.3), (2.4)), then it follows easily from $S U(1 ; 1)$ covariance of the net and $S U(1 ; 1)$-invariance of the vacuum vector that

$$
\begin{gathered}
\pi_{0}(A) J_{(\alpha ; \beta)} \pi_{0}(A)^{*}=J_{(\gamma ; \delta)}, \\
\pi_{0}(A) \Delta_{(\alpha ; \beta)}^{1 / 2} \pi_{0}(A)^{*}=\Delta_{(\gamma ; \delta)}^{1 / 2} .
\end{gathered}
$$

In particular,

$$
\begin{aligned}
\hat{\pi}_{0}\left(I_{(\alpha ; \beta)}\right) & =\pi_{0}\left(N_{(\alpha ; \beta)}\right) J_{S_{+}} \pi_{0}\left(N_{(\alpha ; \beta)}\right)^{*} \\
& =J_{(\alpha ; \beta)} .
\end{aligned}
$$

Notice also that, by duality,

$$
J_{(\alpha ; \beta)}=J_{(\beta ; \alpha)}, \quad \Delta_{(\alpha ; \beta)}^{1 / 2}=\Delta_{(\beta ; \alpha)}^{-1 / 2},
$$

so that by functional calculus,

$$
\Delta_{(\alpha ; \beta)}^{i t}=\Delta_{(\beta ; \alpha)}^{-i t} .
$$

Since $S U(1 ; 1)$ is generated by products of reflections and $\hat{\pi}_{0}\left(I_{(\alpha ; \beta)}\right)=J_{(\alpha ; \beta)}$, it follows that the representation $\pi_{0}$ of $S U(1 ; 1)$ is entirely determined by the modular structure of the net, that is, by the vacuum state and the net. Uniqueness follows from the uniqueness of the modular conjugations with respect to $\Omega$. This completes the proof of (iii).

Ad (iv). It follows from (2.8), (2.9) and (2.10) that

$$
\Delta_{S_{+}}^{i t} \pi_{0}(A) \Delta_{S_{+}}^{-i t}=\pi_{0}\left(D(t) A D(t)^{-1}\right), \quad \forall A \in S U(1 ; 1) .
$$

Since the equation

$$
\Delta_{S_{+}}^{\imath t} J_{S_{+}} \Delta_{S_{+}}^{-\imath t}=J_{S_{+}}
$$

may be written as

$$
\Delta_{S_{+}}^{i t} \hat{\pi}_{0}\left(I_{S_{+}}\right) \Delta_{S_{+}}^{-i t}=\hat{\pi}_{0}\left(I_{S_{+}}\right)
$$

it follows that

$$
\Delta_{S_{+}}^{i t} \hat{\pi}_{0}(A) \Delta_{S_{+}}^{-i t}=\hat{\pi}_{0}\left(D(t) A D(t)^{-1}\right), \quad \forall A \in S U(1 ; 1)_{ \pm} .
$$

In particular,

$$
\begin{aligned}
\Delta_{S_{+}}^{\imath t} J_{(-i ; i)} \Delta_{S_{+}}^{-\imath t} & =\Delta_{S_{+}}^{i t} \hat{\pi}_{0}\left(I_{(-i ; i)}\right) \Delta_{S_{+}}^{-i t} \\
& =\hat{\pi}_{0}\left(D(t) I_{(-i ; i)} D(t)^{-1}\right) \\
& =\hat{\pi}_{0}\left(I_{(D(t)(-i) ; D(t) i)}\right)
\end{aligned}
$$

holds. 
We temporarily assume that we have proven the following relation:

$$
J_{(-i ; i)} \Delta_{S_{+}}^{2 t}=\Delta_{S_{+}}^{-i t} J_{(-i ; i)} .
$$

Equation (2.15) implies that

$$
\Delta_{S_{+}}^{i t} J_{(-i ; i)} \Delta_{S_{+}}^{-\imath t}=\Delta_{S_{+}}^{2 i t} J_{(-i ; i)}
$$

and, plugging this in Eq. (2.14), we obtain

$$
\Delta_{S_{+}}^{2 i t}=J_{(D(t)(-i) ; D(t)(i))} J_{(-i ; i)}=\pi_{0}(D(2 t)),
$$

where the last equality follows from the representation of dilatations as products of reflections (see Appendix I, Eq. (AI.6)). This completes the proof of (iv) provided Eq. (2.15) can be verified.

It follows from Eq. (2.12) and functional calculus that if $A \in S U(1 ; 1)$ and $I=A \cdot S_{+} \subset S^{1}$ then

$$
\pi_{0}(A) \Delta_{S_{+}}^{i t} \pi_{0}(A)^{*}=\Delta_{I}^{2 t}, \quad \forall t \in \mathbb{R}
$$

Now, let $A:=R(-1 / 4)=\left(\begin{array}{cc}e^{-i \pi / 4} & 0 \\ 0 & e^{i \pi / 4}\end{array}\right)$. One checks by direct computation that $A \cdot S_{+}=(-i ; i)$ and that

$$
I_{(-i ; i)} \cdot A^{-1}=A \cdot I_{(-i ; i)} .
$$

From Eq. (2.16) it follows that

$$
\pi_{0}(A) \Delta_{S_{+}}^{\imath t} \pi_{0}(A)^{*}=\Delta_{A \cdot S_{+}}^{i t}=\Delta_{(-i ; i)}^{i t},
$$

and hence

$$
\begin{aligned}
J_{(-i ; i)} \Delta_{S_{+}}^{i t} & =J_{(-i ; \imath)} \pi_{0}\left(A^{-1}\right) \Delta_{(-i ; i)}^{2 t} \pi_{0}(A) \\
& =\hat{\pi}_{0}\left(I_{(-i ; i)} \cdot A^{-1}\right) \Delta_{(-i ; \imath)}^{i t} \pi_{0}(A) \\
& =\hat{\pi}_{0}\left(A I_{(-i ; i)}\right) \Delta_{(-i ; i)}^{2 t} \pi_{0}(A) \\
& =\hat{\pi}_{0}(A) J_{(-i ; i)} \Delta_{(-i ; i)}^{i t} \pi_{0}(A) \\
& =\pi_{0}(A) \Delta_{(-i ; i)}^{i t} J_{(-i ;)} \pi_{0}(A) \\
& =\pi_{0}(A) \Delta_{(-i ; i)}^{2 t} \pi_{0}\left(A^{-1}\right) J_{(-i ; \imath)}
\end{aligned}
$$

where we used Eqs. (2.17) and (2.18) twice.

But

$$
\begin{aligned}
\pi_{0}(A) \Delta_{(-i ; i)}^{i t} \pi_{0}\left(A^{-1}\right) & =\Delta_{A \cdot(-i ; i)}^{i t} \\
& =\Delta_{(-1 ; 1)}^{2 t} \\
& =\Delta_{(1 ;-1)}^{-\imath t}
\end{aligned}
$$

where we used Eq. (2.13) to obtain the last equality. Inserting the last equation in (2.19) we obtain

$$
J_{(-i ; i)} \Delta_{S_{+}}^{\imath t}=\Delta_{S_{+}}^{-i t} J_{(-i ; i)},
$$

and this completes the proof of the theorem.

The result obtained in the previous theorem can be summarized by saying that if the spectrum of the generator of translations of the representation $\pi_{0}$ is positive then 
the one-parameter group of dilatations $\pi_{0}(D(t))$ coincides with the modular group of the algebra $\mathscr{A}=\mathscr{A}\left(S_{+}\right)$with respect to $\Omega$. Another simple application of the results of Borchers [34] shows that the converse is also true:

Theorem 2.20. Let $\left\{\mathscr{H} ; \pi_{0} ; \mathscr{b} ; \Omega\right\}$ satisfy all properties of Definition 2.5, except for the spectrum condition and let $\{\mathscr{b}(I)\}_{I \subset S^{1}}$ be the corresponding conformal net. If the one-parameter subgroup of dilatations coincides with the modular group of the algebra $\mathscr{b}\left(S_{+}\right)$with respect to the vacuum state then the generator of translations has positive spectrum.

Proof. Let $V(p):=\pi_{0}(T(p))$ denote the one-parameter group of translations on $\mathscr{H}$. Since by assumption $\Delta_{S_{+}}^{i t}=\pi_{0}(D(t))$, the following commutation relation holds between $\Delta_{S_{+}}^{i t}$ and $V(p)$ :

$$
\begin{aligned}
\Delta_{S_{+}}^{i t} V(p) \Delta_{S_{+}}^{-i t} & =\pi_{0}(D(t)) \pi_{0}(T(p)) \pi_{0}\left(D(t)^{-1}\right) \\
& =\pi_{0}\left(T\left(e^{-2 \pi t} p\right)\right), \quad \forall t, p \in \mathbb{R} .
\end{aligned}
$$

Let now $p$ be positive and fixed. Since ad $V(p)$ maps $\mathscr{A}\left(S_{+}\right)$into $\mathscr{A}\left(S_{+}\right)$, Lemma II.3 of ref. [34] implies that the function

$$
t \mapsto \Delta_{S_{+}}^{\imath t} V(p) \Delta_{S_{+}}^{-\imath t}
$$

has an analytic extension to the $\operatorname{strip} S(-1 / 2 ; 0)=\{z \in \mathbb{C} \mid-1 / 2<\operatorname{Im} z<0\}$ as an operator-valued function and that furthermore, on $\overline{S(-1 / 2,0)}$, the bound

$$
\left\|\Delta_{S_{+}}^{i z} V(p) \Delta_{S_{+}}^{-i z}\right\| \leq 1
$$

holds uniformly in $z$. From Eq. (2.20) we conclude that the function

$$
t \mapsto V\left(e^{-2 \pi t} p\right)
$$

has an analytic extension to complex $t$ 's contained in the strip $S(-1 / 2 ; 0)$ and the bound

$$
\left\|V\left(e^{-2 \pi z} p\right)\right\| \leq 1
$$

holds for all $z \in S(-1 / 2 ; 0)$. Going over to the variable $y:=e^{-2 \pi t} \cdot p$ we see that the domain $S(-1 / 2 ; 0)$ is transformed into

$$
\mathbb{C}^{+}=\{w \in \mathbb{C} \mid \operatorname{Im} w>0\}
$$

so that, as a function of $y$,

$$
y \mapsto V(y)
$$

has an analytic extension into the upper half-plane $\mathbb{C}^{+}$, and on $\overline{\mathbb{C}^{+}}$it satisfies the bound $\|V(w)\| \leq 1$.

Let

$$
V(y)=\int_{-\infty}^{+\infty} e^{i p y} d E(p)
$$


be the spectral decomposition of $V(y)$. Assume that $E\left(p_{0}\right) \neq 0$ for some $p_{0}<0$. Then there exists a vector $\psi \in \mathscr{H}$ such that $E\left(p_{0}\right) \psi=\psi$ and $\|\psi\|=1$. If $w=y+i x$, $x \geq 0$ we can estimate the norm of $V(w) \psi$ as follows:

$$
\begin{aligned}
\|V(w) \psi\|^{2} & =\int_{-\infty}^{p_{0}} e^{-2 x p} d\|E(p) \psi\|^{2} \\
& \geq e^{-2 x p_{0}} \int_{-\infty}^{p_{0}} d\|E(p) \psi\|^{2} \geq e^{-2 x p_{0}} .
\end{aligned}
$$

If $x \rightarrow \infty$ the right-hand side of this inequality can be made arbitrarily large, contradicting the bound $\|V(w)\| \leq 1$. Hence $E(p)=0, \forall p<0$, that is, the spectrum of the generator of translations is contained in $\{0\} \cup \mathbb{R}^{+}$. This completes the proof of the theorem.

\section{II.3. Local Internal Symmetries of Vacuum Sectors}

In this section, we complete our description of the relation between the modular structure of the local algebras in the vacuum sector of a conformal field theory and the representation $\pi_{0}$ of the Moebius group by constructing the modular conjugation $J_{S_{+}}$from the representation $\pi_{0}$ of $S U(1 ; 1)$ and by identifying the group of local internal symmetries of the net. The proofs of the following results, which are of a technical nature, are relegated to Appendix II.

Definition 2.21. Let $\left\{\mathscr{H} ; \pi_{0} ; \mathscr{A} ; \Omega\right\}$ be the vacuum sector of a conformal field theory.

(i) The group $\mathscr{G} \subseteq \mathscr{U}(\mathscr{H})$ of unitaries such that

$$
U . \not C(I) U^{*}=\mathscr{C}(I), \quad \forall I \subset S^{1}, \quad U \Omega=\Omega,
$$

is called the group of local internal symmetries of the vacuum sector. ${ }^{5}$

(ii) Let $\hat{\pi}_{0}$ be the corepresentation of $S U(1 ; 1)_{ \pm}$constructed in Theorem 2.19 . We set

$$
\tilde{\mathscr{G}}:=\left\{U \in \mathscr{B}(\mathscr{H}) \mid U \hat{\pi}_{0}(A)=\hat{\pi}_{0}(A) U, \forall A \in S U(1 ; 1)_{ \pm} ; \text {and } U \Omega=\Omega\right\} .
$$

Lemma 2.22. Let $\left\{\mathscr{H} ; \pi_{0} ; \mathscr{b} ; \Omega\right\}$ be the vacuum sector of a conformal field theory. Then $\mathscr{G} \subseteq \tilde{\mathscr{G}}$.

Proof. See Appendix II.

Definition 2.23. Let $\left\{\mathscr{H} ; \pi_{0} ; \mathscr{b} ; \Omega\right\}$, be the vacuum sector of a conformal field theory. For $U \in \tilde{\mathscr{G}}$ we define

$$
\mathscr{C}^{U}(I):=\left\{A \in \mathscr{C}(I) \mid U A U^{*} \in \mathscr{C}(I)\right\}, \quad \forall I \subset S^{1} .
$$

The net $\left\{\mathscr{C}^{U}(I)\right\}_{I \subset S^{1}}$ is a local conformal net contained in $\{\mathscr{C}(I)\}_{I \subset S^{1}}$.

The following criteria identifies elements of $\mathscr{G}$ in $\tilde{\mathscr{G}}$.

\footnotetext{
5 This group was introduced in ref. [56], see also [37]
} 
Lemma 2.24. Let $\left\{\mathscr{H} ; \pi_{0} ; \mathscr{b} ; \Omega\right\}$ be the vacuum sector of a conformal field theory. Then $U \in \mathscr{G}$ if and only if $U \in \tilde{\mathscr{G}}$ and the vacuum vector $\Omega$ is cyclic for the von Neumann algebra $\mathscr{B}:=\left\{U_{I \subset S^{1}} \mathscr{b}^{U}(I)\right\}^{\prime \prime}$ generated by the net $\left\{\mathscr{C}^{U}(I)\right\}_{I \subset S^{1}}$.

Proof. See Appendix II.

Definition 2.25. Let $\left\{\mathscr{H}_{i} ; \pi_{0}^{i} ; \mathscr{A}_{i} ; \Omega_{i}\right\}, i=a, b$, be two vacuum sectors of conformal field theories. These two sectors are said to be equivalent if there exists an invertible isometry $V: \mathscr{H}_{a} \mapsto \mathscr{H}_{b}$ such that

$$
\begin{aligned}
V \pi_{0}^{a}(\cdot) & =\pi_{0}^{b}(\cdot) V, \\
V \Omega_{a} & =\Omega_{b}, \\
V \mathscr{A}_{a} V^{*} & =\mathscr{C}_{b} .
\end{aligned}
$$

Definition 2.26. Let $\mathscr{H}_{1}, \mathscr{H}_{2}$ be two Hilbert spaces, $e=\left\{e_{1}, \ldots e_{n}, n=\operatorname{dim} \mathscr{H}_{1}\right\}$ an orthonormal basis in $\mathscr{H}_{1}$ and $A$ an antilinear operator in $\mathscr{H}_{2}$. Each vector $\varphi \in \mathscr{H}_{1} \otimes \mathscr{H}_{2}$ can be written uniquely as

$$
\varphi=\sum_{i=1}^{\operatorname{dim} \mathscr{H}_{1}} e_{i} \otimes \varphi_{i}, \quad \varphi_{i} \in \mathscr{H}_{2} .
$$

(i) The conjugation $J(e)$ associated to the basis $e$ on $\mathscr{H}_{1}$ is the unique antilinear extension of

$$
J(e) e_{i}=e_{i} \quad i=1, \ldots \operatorname{dim} \mathscr{H}_{1} .
$$

(ii) A linear operator $B \in \mathscr{B}\left(\mathscr{H}_{1}\right)$ is said to be real with respect to the basis e of $\mathscr{H}_{1}$, if

$$
J(e) B J(e)=B .
$$

(iii) The antilinear extension of $A$ with respect to $e$ on $\mathscr{H}_{1} \otimes \mathscr{H}_{2}$ is the unique antilinear map $\hat{A}(e)$ defined by

$$
\hat{A}(e) \varphi:=\sum_{i=1}^{\operatorname{dim} \mathscr{H}_{1}} e_{i} \otimes A \varphi_{i}, \quad \varphi=\sum_{i=1}^{\operatorname{dim} \mathscr{H}_{1}} e_{i} \otimes \varphi_{i} .
$$

Lemma 2.27. Let $\left(\pi ; \mathscr{H}_{\pi}\right)$ be an irreducible representation of $S U(1 ; 1)$ belonging to the holomorphic discrete series. Then $\pi$ extends uniquely to an irreducible corepresentation of $S U(1 ; 1)_{ \pm}$. That is, there exists on $\mathscr{H}_{\pi}$ a (up to a phase factor) unique conjugation $J_{\pi}$ such that

$$
J_{\pi}^{2}=\mathbb{1}, \quad J_{\pi} \pi(A) J_{\pi}=\pi(\bar{A}), \quad \forall A \in S U(1 ; 1)
$$

holds.

Proof. See Appendix II.

Theorem 2.28. (Structure of the vacuum sector of a conformal field theory.) Let $\left\{\mathscr{H} ; \pi_{0} ; \mathscr{A} ; \Omega\right\}$ be the vacuum sector of a conformal field theory. Then $\left\{\mathscr{H} ; \pi_{0} ; \mathscr{A}\right.$; $\Omega\}$ is equivalent to a vacuum sector $\left\{\mathscr{H}^{\prime} ; \pi_{0}^{\prime} ; \mathscr{b}^{\prime} ; \Omega^{\prime}\right\}$ having the following properties:

(i) $\mathscr{H}^{\prime}=\mathscr{H}_{\Omega^{\prime}} \oplus \bigoplus_{i=1}^{\infty}\left(\mathscr{H}_{i} \otimes \mathscr{H}_{\pi^{i}}\right)$, where $\mathscr{H}_{\Omega^{\prime}}:=\left\{\mathbb{C} \Omega^{\prime}\right\}, \operatorname{dim} \mathscr{H}_{i}=i$. 
(ii) $\pi_{0}^{\prime}=\mathbb{1}_{1} \otimes \pi^{1} \oplus \mathbb{1}_{2} \otimes \pi^{2} \oplus \cdots \oplus \mathbb{1}_{\infty} \otimes \pi^{\infty}$, where the $\pi^{i}$ 's are disjoint, multiplicityfree direct sums of holomorphic discrete series representations ${ }^{6}$ of $S U(1 ; 1)$ on $\mathscr{H}_{\pi^{2}}$ : $\pi^{i}=\bigoplus_{\alpha} \pi^{i, \alpha}$, and each $\pi^{i, \alpha}$ is irreducible.

(iii) The modular conjugation $J_{S_{+}}^{\prime}$ of $\mathscr{A}^{\prime}\left(S_{+}\right)=\mathcal{A}^{\prime}$ is given by

$$
J_{S_{+}}^{\prime}=\hat{\pi}_{0}^{\prime}\left(I_{S_{+}}\right)=\hat{J}_{\pi^{1}}\left(e^{1}\right) \oplus \hat{J}_{\pi^{2}}\left(e^{2}\right) \oplus \cdots \oplus \hat{J}_{\pi^{\infty}}\left(e^{\infty}\right)
$$

where $e^{i}=\left\{e_{1}^{i} ; e_{2}^{i} ; \ldots e_{i}^{i}\right\}$ are orthonormal bases of $\mathscr{H}_{i}, i=1,2, \ldots \infty$, and $J_{\pi^{i}}=\bigoplus_{\alpha} J_{\pi^{2}, \alpha}$. The conjugations $J_{\pi^{i, \alpha}}$ are those constructed in Lemma 2.27.

(iv) If an operator $U \in \mathscr{Q} b\left(\mathscr{H}^{\prime}\right)$ is a local internal symmetry of the vacuum sector $\left\{\mathscr{H}^{\prime} ; \pi_{0}^{\prime} ; A^{\prime} ; \Omega^{\prime}\right\}$, then

$$
U=\mathbb{1}_{\Omega^{\prime}} \oplus \bigoplus_{i=1}^{\infty} U_{i} \otimes \mathbb{1}_{\mathscr{H}_{\pi^{2}}}
$$

with each $U_{i}$ unitary in $\mathscr{H}_{i}$ and real with respect to the orthonormal basis $e^{\imath}$ of $\mathscr{H}_{i}$, $i=1, \ldots \infty$.

Proof. See Appendix II.

In view of the previous results, we are led to believe that under appropriate assumptions, some version of the following conjecture is true.

Conjecture 2.29.

(i) Given a local, isotone and dual net $I \rightarrow \mathscr{A}_{(}(I)$ of hyperfinite type $I I I_{1}$ factors in $\mathscr{B}(\mathscr{H} b)$ indexed by intervals of the circle and a vector $\Omega$ cyclic and separating for each . $\ell_{(}(I), I \subset S^{1}$, one can construct, for each interval $I$, the modular group $\Delta_{i}^{2 t}$ and the modular conjugation $J_{I}$ of the algebra $\mathscr{A}(I)$. These correspond to the one-parameter groups of Moebius transformations leaving the end points of the interval $I$ fixed and to the inversion about the end points of $I$ (see Theorem 2.19). It should be possible to verify that these one-parameter groups and inversions form a representation of $S U(1 ; 1)_{ \pm}$under which the net transforms covariantly. To simplify the task, one could assume the existence of the one-parameter group of rotations satisfying the spectrum condition.

(ii) Given the vacuum sector of a conformal field theory $\left\{\mathscr{H} ; \pi_{0} ; \mathscr{t} ; \Omega\right\}$, one expects an algebraic version of the Lüscher-Mack theorem [1] to be true. Formally, it is possible to define an energy-momentum tensor $T(z)$ as an $h=2$ coefficient of the expansion

$$
\begin{aligned}
& \alpha_{t}(A)=(\Omega ; A \Omega) \mathbb{1}+\sum_{h \in \mathbb{Z}_{+}} t^{-h} \phi_{A}^{(h)}(z)(t \rightarrow \infty), \\
& \phi_{A}^{(2)}(z) \propto T(z),
\end{aligned}
$$

where $A$ is a suitable local observable and $\alpha_{t}(A):=\operatorname{ad} \pi_{0}(K(t))(A)$ for the oneparameter group $K(t)$ of $P S U(1 ; 1)$ which contracts intervals to the point $z \in S^{1}$ (for example, $K(t)=D(t)$ for $z=1$ ). The Fourier coefficients of $T(z)$ should then satisfy the commutation relations of the Virasoro algebra.

(iii) Another possible way of deriving the Lüscher-Mack theorem is to assume the theory to be supersymmetric in the following sense. Let $\mathscr{H}$ be a separable Hilbert space, $\pi_{0}$ a unitary representation of the Moebius group on $\mathscr{H}$ leaving a vector $\Omega$

\footnotetext{
6 possibly trivial
} 
invariant, and $\mathscr{t} 6$ a von Neumann algebra transforming properly under $\pi_{0}$. We use the standard notation, $L_{-1}, L_{0} \equiv K, L_{+1}$, for the infinitesimal generators of the Moebius group. We now assume the existence of an operator $G_{0}$ on $\mathscr{H}$ such that

$$
G_{0}^{2}=L_{0}-\frac{c}{24} \cdot \mathbb{1}_{\mathscr{H}}, \quad G_{0}=G_{0}^{*}
$$

and of a unitary involution $\Gamma$ such that

$$
\begin{aligned}
& \Gamma A=A \Gamma, \quad \forall A \in \mathcal{A}, \quad \Gamma \Omega=\Omega, \\
& \Gamma G_{0}=-G_{0} \Gamma, \quad \Gamma L_{\imath}=L_{\imath} \Gamma, \quad i=0,-1,1 .
\end{aligned}
$$

This implies that $\mathscr{H}$ is the direct sum of the eigenspaces of $\Gamma$,

$$
\mathscr{H}=\mathscr{H}_{+} \oplus \cdot \mathscr{H}_{-},\left.\quad \Gamma\right|_{\mathscr{H}_{+}}=\left.\mathbb{1}\right|_{\mathscr{H}_{+}},\left.\quad \Gamma\right|_{\mathscr{H}_{-}}=-\left.\mathbb{1}\right|_{\mathscr{H}_{-}}
$$

and that

$$
\overline{\mathscr{A} \Omega} \subseteq \mathscr{H}_{+} .
$$

By defining inductively

$$
\begin{aligned}
G_{1} & :=2\left[L_{1}, G_{0}\right], & G_{-1} & :=2\left[L_{-1}, G_{0}\right], \\
L_{2} & :=\frac{1}{2}\left[G_{1}, G_{1}\right]_{+} & L_{-2} & :=\frac{1}{2}\left[G_{-1}, G_{-1}\right]_{+}, \ldots, \\
G_{n} & :=\frac{1}{2}\left[L_{n}, G_{0}\right], & G_{-n} & :=2\left[L_{-n}, G_{0}\right], \\
L_{n+1} & :=\frac{1}{2}\left[G_{n}, G_{1}\right]_{+}, & L_{-(n+1)} & :=\left[G_{-n}, G_{-1}\right]_{+}, \quad n \in \mathbb{Z}_{+},
\end{aligned}
$$

where $[\cdot, \cdot]_{+}$denotes the anticommutator, we expect to obtain from $L_{-1}, L_{0}, L_{1}$ and $G_{0}$ a Ramond algebra specified by the commutation relations

$$
\begin{aligned}
{\left[L_{m}, L_{n}\right] } & =(m-n) L_{m+n}+\frac{c}{12} m\left(m^{2}-1\right] \delta_{m+n, 0}, \\
{\left[L_{m}, G_{n}\right] } & =\left(\frac{1}{2} m-n\right) G_{m+n}, \\
{\left[G_{m}, G_{n}\right]_{+} } & =2 L_{m+n}+\frac{c}{3}\left(m^{2}-1 / 4\right) \delta_{m+n, 0},
\end{aligned}
$$

having the correct grading

$$
\Gamma L_{\imath}=L_{i} \Gamma, \quad i \in \mathbb{Z}, \quad \Gamma G_{i}=-G_{i} \Gamma, \quad i \in \mathbb{Z}
$$




\section{The Conformal Nets Associated to Positive-Energy Representations of Loop Groups}

As an application of the results presented in Chap. II, we construct conformal nets in positive-energy representations of certain loop groups. Some of these nets will be shown to be nets in the vacuum sector of a conformal field theory in the sense of Chap. II. The analysis of their superselection structure will be sketched in the next chapter. Algebraic quantum field theories of this kind were already considered by Buchholz, Mack and Todorov [30] for the loop group $L T$ of the one-dimensional torus $T$, in order to classify quantum field theories having the $U(1)$-current algebra as a common germ. Indeed, some of the main ingredients of our construction were already mentioned in [30]. Results similar to those presented here have also been announced by Wassermann [29].

In the following sections, we gather all the necessary ingredients scattered through the literature.

\section{III.1. Simple, Simply Laced Lie Algebras and Groups}

Let $G_{0}$ be a simple, simply connected and simply laced compact Lie group. The Lie algebra, $\mathscr{G}_{0}$, of $G_{0}$ belongs to the $A, D$ or $E$ series. Let $\mathscr{G}$ be the complexification of $\mathscr{G}$. Fix a Cartan subalgebra $\mathfrak{h} \subseteq \mathscr{G}$ and let $\Delta$ denote the roots of $\mathfrak{h}$ on $\mathscr{G}$. We then have the direct sum decomposition

$$
\mathscr{G}=\mathfrak{h} \bigoplus_{\alpha \in \Delta} \mathscr{G}_{\alpha}
$$

where $\mathscr{G}_{\alpha}$ denote the one-dimensional root spaces. Choose a set of positive roots, $\Delta_{+}$, in $\Delta$, let $\pi=\left\{\alpha_{1}, \ldots \alpha_{l}, l=\operatorname{dim} \mathfrak{h}\right\}$ be the set of simple roots and let $\theta$ be the highest root in $\Delta_{+}$. Choose a non-degenerate, invariant symmetric bilinear form $(\cdot \mid \cdot)$ on $\mathscr{G}$, normalized as follows: the restriction of $(\cdot \mid \cdot)$ to $\mathfrak{h}$ is non-degenerate, hence induces an isomorphism $\nu: \mathfrak{h} \rightarrow \mathfrak{h}^{*}$ and a non-degenerate bilinear form $(\cdot \mid \cdot)$ on $\mathfrak{h}^{*}$. We require that

$$
(\theta \mid \theta)=2
$$

Then $(\alpha \mid \alpha)=2$, for any root $\alpha$ in $\Delta$, since all roots of a simply laced Lie algebra are long. Given a root $\alpha$, let $h_{\alpha}:=\nu^{-1}(\alpha) \in \mathfrak{h}$ be the corresponding coroot. We have that

$$
\theta=\sum_{i=1}^{l} a_{i} \cdot \alpha_{i}, \quad h_{\theta}=\sum_{i=1}^{l} a_{i} \cdot h_{\alpha_{\imath}}
$$

where the integers $a_{\imath}$ are positive and are given in Table I below. The number $g:=1+\sum_{i=1}^{l} a_{i}$ is the dual Coxeter number of $\mathscr{G}$.

Let $\Lambda_{\imath} \in \mathfrak{h}^{*}, i=1, \ldots l$, be the fundamental weights of $\mathscr{G}$ defined by $\Lambda_{i}\left(h_{\alpha_{\jmath}}\right)=$ $\delta_{i j}, j=1, \ldots l$. Define $\varrho:=\sum_{i=1}^{l} \Lambda_{i}$. 
Table I.

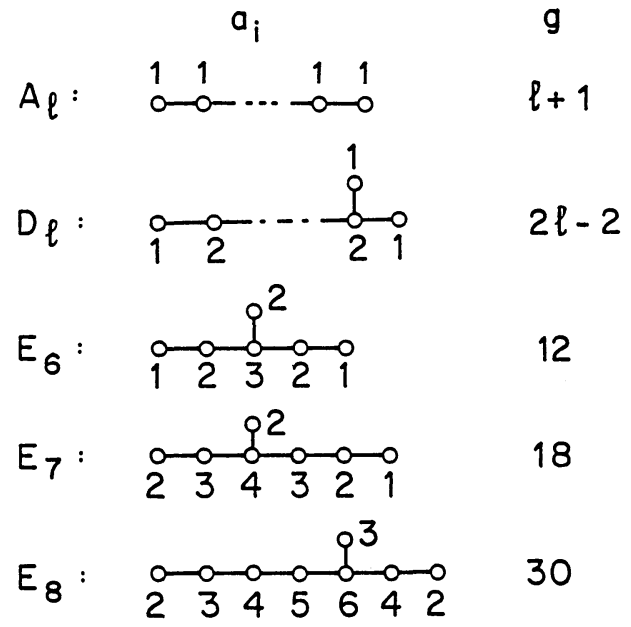

If we pick a Chevalley basis $\left\{e_{\alpha}\right\}_{\alpha \in \Delta} \cup\left\{h_{\alpha_{\imath}}, i=1, \ldots l\right\}$ of $\mathscr{G}$ then the real span of

$$
\left\{i h_{\alpha} ; e_{\alpha}-e_{-\alpha} ; i\left(e_{\alpha}+e_{-\alpha}\right)\right\}_{\alpha \in \Delta}
$$

is a compact real form $\mathscr{G}_{k}$ of $\mathscr{G}$ which is isomorphic to $\mathscr{G}_{0}$. We will identify $\mathscr{G}_{0}$ with $\mathscr{G}_{k}$ in the following. Let $\tau$ be the conjugation of $\mathscr{G}$ defined by the compact real form $\mathscr{G}_{k}$ and set $x^{*}:=-\tau(x)$ for $x \in \mathscr{G}$. Then

$$
\mathscr{G}_{k}=\left\{x \in \mathscr{G} \mid x^{*}=-x\right\} \text {. }
$$

Furthermore, $\left(x \mid y^{*}\right)$ is a positive Hermitian form on $\mathscr{G}$. Later on, we will need a pair of dual bases of $\mathscr{G}$, i.e., two bases $\left\{u_{i}\right\}$ and $\left\{u^{\jmath}\right\}$ of $\mathscr{G}$ such that $\left(u_{i} \mid u^{\jmath}\right)=\delta_{i j}$.

\section{III.2. The Affine Lie Algebra $\hat{\mathscr{G}}$ Associated with $\mathscr{G}$}

Let $\mathbb{C}\left[t ; t^{-1}\right]$ be the algebra of Laurent polynomials in $t$. The residue of a Laurent polynomial $f=\sum_{k \in \mathbb{Z}} c_{k} t^{k}$ (where all but a finite number of $c_{k}$ are zero) is defined by Res $f=c_{-1}$. Res is a linear functional on $\mathbb{C}\left[t ; t^{-1}\right]$ characterized by the properties

$$
\operatorname{Res} t^{-1}=1, \quad \operatorname{Res} \frac{d f}{d t}=0 .
$$

If we define

$$
\tilde{\mathscr{G}}:=\mathscr{G} \otimes_{\mathbb{C}} \mathbb{C}\left[t ; t^{-1}\right]
$$

then $\tilde{\mathscr{G}}$ is a Lie algebra under the obvious bracket operation. $\tilde{\mathscr{G}}$ can be viewed as a subalgebra of the rational maps from $\mathbb{C}^{\times}$to $\mathscr{G}$. The evaluation of $x \in \tilde{\mathscr{G}}$ on $S^{1}$ is then obtained by replacing $t$ by $e^{i \theta}$. We will often use the convenient notation $x(n)$ for $x \otimes t^{n}, x \in \mathscr{G}$ and identify $\mathscr{G}$ with $\mathscr{G} \otimes 1$ in $\tilde{\mathscr{G}}$.

Let $\Omega$ be the bilinear form on $\tilde{\mathscr{G}}$ defined by

$$
\Omega(f ; g):=\operatorname{Res}\left(\frac{d f}{d t} \mid g\right),
$$


where (.|.) is the invariant symmetric bilinear form of Sect. III.1. Thus, $\Omega(x(n)$; $y(m))=n \delta_{n,-m}(x \mid y)$ for $x, y \in \mathscr{G}$. One easily checks that $\Omega$ is a two-cocycle on $\tilde{\mathscr{G}}$, and we denote by $\hat{\mathscr{G}}$ the corresponding central extension of $\tilde{\mathscr{G}}$ :

$$
0 \rightarrow \mathbb{C} \rightarrow \hat{\mathscr{G}} \rightarrow \tilde{\mathscr{G}} \rightarrow 0 .
$$

As a vector space, we take $\hat{\mathscr{G}}=\tilde{\mathscr{G}} \oplus \mathbb{C} \cdot c$ with the commutation relations

$$
[f, g]=[f, g]_{0}+\Omega(f ; g) \cdot c,
$$

for $f, g \in \tilde{\mathscr{G}}$. Here [, ] denotes the bracket in $\tilde{\mathscr{G}}$, and $c$ is central. For $x, y \in \mathscr{G}$, we have

$$
[x(m), y(n)]=[x ; y](m+n)+m \delta_{m,-n}(x \mid y) c .
$$

$\hat{\mathscr{G}}$ is the affine Lie algebra associated to $\mathscr{G}$.

The map $x \mapsto x^{*}, x \in \mathscr{G}$, extends to a conjugate linear anti-automorphism of $\hat{\mathscr{G}}$ by defining $c^{*}=c$ and $x(n)^{*}=x^{*}(-n), x \in \mathscr{G}, n \in \mathbb{Z}$.

Let $\hat{\mathscr{G}}_{k}:=\left\{x \in \hat{\mathscr{G}} \mid x^{*}=-x\right\}, \hat{\mathscr{G}}_{k}$ is a real form of $\hat{\mathscr{G}}$, the so-called "compact form."

\section{III.3. The Loop Group $L G_{0}$ and its Central Extension $\widehat{L G}_{0}$}

We denote by $L G_{0}$ the group of smooth maps $f: S^{1} \rightarrow G_{0}$ under pointwise multiplication. $L G_{0}$ can be given the structure of a Fréchet Lie group [57]. The Lie algebra of $L G_{0}$ consists of smooth maps $f: S^{1} \rightarrow \mathscr{G}_{0}$, with the bracket operation induced by $\mathscr{G}_{0}$. We denote by $L \mathscr{G}_{0}^{\text {pol }}$ the subalgebra of $L \mathscr{G}_{0}$ consisting of loops having finite Fourier series.

We define a skew-symmetric, bilinear form $\omega$ on $L \mathscr{G}_{0}$ by

$$
\omega(f ; g)=\frac{1}{2 \pi} \int_{0}^{2 \pi}\left(f(\theta) \mid g^{\prime}(\theta)\right) d \theta,
$$

where $(\cdot \mid \cdot)$ is the invariant symmetric bilinear form of Sect. III.1. One checks that $\omega$ is a two-cocycle on $L \mathscr{G}_{0}$ and that the central extension $\widehat{L \mathscr{G}}_{0}^{\text {pol }}:=L \mathscr{G}_{0}^{\text {pol }} \oplus \mathbb{R} \cdot i c$ defined by this cocycle coincides with $\hat{\mathscr{G}}_{k}$ when evaluated on $S^{1}$. Hence $\hat{\mathscr{G}}$ is the complexified Lie algebra of $\widehat{L \mathscr{G}}_{0}^{\text {pol }}$.

The corresponding central extension $\widehat{L \mathscr{G}}_{0}$ of $L \mathscr{G}_{0}$ given by the cocycle $\omega$ lifts [58] to a central extension of the loop group $L G_{0}$ :

$$
1 \rightarrow T \rightarrow \widehat{L G}_{0} \stackrel{p}{\rightarrow} L G_{0} \rightarrow 1 .
$$

However, since $\widehat{L G}_{0}$ is a non-trivial $U(1)$-bundle over $L G_{0}$, this extension cannot be globally defined by a continuous Lie group cocycle. A convenient description of $\widehat{L G}_{0}$ has been given by Mickelsson $[59,60]$ by considering $\widehat{L G}_{0}$ as a quotient of a larger, trivial $U(1)$-bundle.

Let $D=\{z \in \mathbb{C}|| z \mid \leq 1\}$ be the unit disc in $\mathbb{C}$ and $D G_{0}$ the group of smooth maps from $D$ into $G_{0}$ with radial derivative vanishing to all orders at the boundary. 
Denote by $\mathscr{G}_{n}$ the normal subgroup of $D G_{0}$ consisting of maps $f: D \rightarrow G_{0}$ such that $\left.f\right|_{S^{1}} \equiv e$, where $e \in G_{0}$ denotes the unit element.

We define a real-valued two-cocycle $\gamma: D G_{0} \times D G_{0} \rightarrow \mathbb{R}$ by

$$
\begin{aligned}
\gamma(f ; g) & =\frac{(\theta \mid \theta)}{16 \pi^{2}} \int_{D}\left(f^{-1} d f \mid d g g^{-1}\right) \\
& =\frac{1}{8 \pi^{2}} \int_{D}\left(f^{-1} d f \mid d g g^{-1}\right)
\end{aligned}
$$

where $(\cdot \mid \cdot)$ is the invariant symmetric bilinear form of Sect. III.1 and a group extension of $D G_{0}$ by $U(1)$ using the multiplication rule

$$
(f ; \lambda) \cdot(g ; \mu)=(f \cdot g ; \lambda \mu \exp 2 \pi i \gamma(f ; g)) .
$$

$\mathscr{G}_{n}$ is embedded in $D G_{0} \times U(1)$ as follows. Let $g \in \mathscr{G}$; since $\left.g\right|_{S^{1}} \equiv e$ we can think of $g$ as a map from $S^{2}$ to $G_{0}$ by identifying the boundary $S^{1}$ of $D$ with the north pole of $S^{2}$. Let $\tilde{g}$ denote an extension of $g$ to $B$, the unit ball in $\mathbb{R}^{3}$ (which exists since $\left.\pi_{2}\left(G_{0}\right)=0\right)$ and define

$$
C(\tilde{g})=\frac{(\theta \mid \theta)}{48 \pi^{2}} \int_{B} \varepsilon^{\alpha \beta \gamma}\left(\tilde{g}^{-1} \partial_{\alpha} \tilde{g} \mid \frac{1}{2}\left[\tilde{g}^{-1} \partial_{\beta} \tilde{g}, \tilde{g}^{-1} \partial_{\gamma} \tilde{g}\right]\right)
$$

One checks that $C(\tilde{g})$ depends on the chosen extension $\tilde{g}$ only modulo $\mathbb{Z}$. Hence the map

$$
\begin{aligned}
\phi: \mathscr{G}_{n} & \rightarrow D G_{0} \times U(1) \\
g & \rightarrow(g ; \exp 2 \pi i C(\tilde{g}))
\end{aligned}
$$

is well-defined and can be shown to be a homomorphism $[59,60]$. The image $\phi\left(\mathscr{G}_{n}\right)$ in $D G_{0} \times U(1)$ is readily seen to be a normal subgroup, and $\widehat{L G}_{0}:=\left(D G_{0} \times U(1)\right) / \phi\left(\mathscr{G}_{n}\right)$ is a circle bundle over the base space $L G_{0}$. The projection is given by

$$
\begin{aligned}
& p: \widehat{L G}_{0} \rightarrow L G_{0} \\
& {\left.[(f ; \lambda)] \rightarrow f\right|_{S^{1}} .}
\end{aligned}
$$

The center of $\widehat{L G}_{0}$ is represented by the pairs $(1, \lambda) \in D G_{0} \times U(1)$. It acts transitively on each fiber, and so $\widehat{L G}_{0}$ is a central extension of $L G_{0}$ by $U(1)$.

The cocycle $\gamma(\cdot ; \cdot)$ induces a Lie algebra cocycle $c(\cdot ; \cdot)$ for the Lie algebra $D \mathscr{G}_{0}$ of $D G_{0}$ which is easily computed. Let $f, g \in D \mathscr{G}_{0}$, then

$$
\begin{aligned}
\frac{1}{2 \pi} c(f ; g) & =\left.2 \frac{d^{2}}{d t d s}\right|_{s=t=0} \gamma\left(e^{t f} ; e^{s g}\right)=\frac{1}{4 \pi^{2}} \int_{D}(d f \mid d g) \\
& =\frac{1}{4 \pi^{2}} \int_{S^{1}}(f \mid d g)
\end{aligned}
$$

so that $c(\cdot ; \cdot)$ coincides with $\omega(\cdot ; \cdot)$.

Finally, let us note that the (Fréchet) Lie group of orientation preserving diffeomorphisms of the circle Diff ${ }^{+}\left(S^{1}\right)$ acts in an obvious way on $L G_{0}$ and that this action lifts to a covering action of $\widehat{L G}_{0}$ [42]. A representation $\left(\pi ; \mathscr{H}_{\pi}\right)$ of $\widehat{L G}_{0}$ is called 
a positive-energy representation if the one-parameter group of rotations acts continuously on $\mathscr{H}_{\pi}$ by unitary operators which implement the rotation automorphisms of $\widehat{L G}_{0}$ and if the generator $L_{0}$ of rotations has positive spectrum.

III.4. The Local Structure of $L G_{0}$ and $\widehat{L G}_{0}$

The description of $\widehat{L G}_{0}$ given in Sect. III.3 allows us to construct local normal subgroups of $\widehat{L G}_{0}$ and of $L G_{0}$.

Let $f: S^{1} \rightarrow G_{0}$ be an element of $L G_{0}$. Denote by supp $f$ the smallest closed subset of $S^{1}$ such that $\left.f\right|_{S^{1} \backslash \operatorname{supp} f} \equiv e$. Define for each open interval $I$ in $S^{1}$

$$
\overline{\mathscr{b}}(I):=\left\{f \in L G_{0} \mid \operatorname{supp} f \subset I\right\}
$$

and

$$
\hat{\mathscr{b}}(I):=p^{-1}(\overline{\mathscr{C}}(I)) \subset \widehat{L G}_{0}
$$

Clearly, $\hat{t}\left(I_{1}\right) \subseteq \hat{\mathscr{b}}\left(I_{2}\right)$ if $I_{1} \subseteq I_{2}$. The following lemma is elementary.

Lemma 3.1. Let $\hat{f} \in \hat{\mathscr{b}}\left(I_{1}\right)$ and $\hat{g} \in \hat{\mathscr{t}}\left(I_{2}\right)$. If $I_{1} \cap I_{2}=\emptyset$ then $\hat{f} \cdot \hat{g}=\hat{g} \cdot \hat{f}$ in $\widehat{L G}_{0}$. Proof. Let $f:=p(\hat{f})$ and $g:=p(\hat{g})$, so that $\operatorname{supp} f \subset I_{1}$ and $\operatorname{supp} g \subset I_{2}$. The construction of $\widehat{L G}_{0}$ given above implies that a representative of $\hat{f}$ in $D G_{0} \times U(1)$ is of the form $(\tilde{f} ; \lambda)$, where $\tilde{f}$ may be constructed from $f$ as follows. Denote by $C_{I_{1}}$ the open cake slice of $D$ having $I_{1}$ and $0 \in D$ on its boundary,

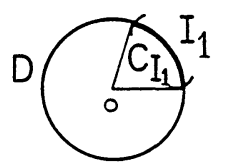

and let $C_{I_{1}}^{\prime}$ be the complement of $C_{I_{1}}$ in $D$. Extend $f$ to a map $\tilde{f}$ on $D$ such that $\left.\tilde{f}\right|_{C_{I_{1}}^{\prime}} \equiv e$. Such an extension exists, since supp $f \subset I_{1}$ and $\pi_{1}\left(G_{0}\right)=0$. Proceed in the same way for $g$. Clearly, $C_{I_{1}} \cap C_{I_{2}}=\emptyset$ since $I_{1} \cap I_{2}=\emptyset$. This implies that

$$
\tilde{f} \cdot \tilde{g}=\tilde{g} \cdot \tilde{f}
$$

and

$$
\gamma(\tilde{f} ; \tilde{g})=0
$$

which in turn means that

$$
(\tilde{f} ; \lambda)(\tilde{g} ; \mu)=(\tilde{g} ; \mu)(\tilde{f} ; \lambda)
$$

holds in $D G_{0} \times U(1)$, so that $\hat{f} \cdot \hat{g}=\hat{g} \cdot \hat{f}$ in $\widehat{L G}_{0}$. This completes the proof of the lemma. 


\section{III.5. The Extension of $\hat{\mathscr{G}}$ by the Virasoro Algebra}

Let $\partial=\mathbb{C}\left[t ; t^{-1}\right] \frac{d}{d t}$ be a subalgebra of the Lie algebra of rational vector fields on $\mathbb{C}$. There is a natural action of $\partial$ as derivations of $\tilde{\mathscr{G}}$ which lifts to $\hat{\mathscr{G}}$ by acting trivially on the central element $c$. In terms of the basis $d_{n}=-t^{n+1} \frac{d}{d t}, n \in \mathbb{Z}$ for $\partial$, one has

$$
d_{n} x(m)=-m x(m+n), \quad d_{n} c=0
$$

for $n, m \in \mathbb{Z}, \in \mathscr{G}$. The commutation relations of $\partial$ are

$$
\left[d_{n}, d_{m}\right]=(n-m) d_{n+m}, \quad n, m \in \mathbb{Z} .
$$

The algebra $\partial$ admits an (essentially unique) non-trivial two-cocycle $\omega$ defined by

$$
\omega\left(d_{n} ; d_{m}\right)=\frac{1}{12}\left(n^{3}-n\right) \delta_{n,-m}, \quad n, m \in \mathbb{Z} .
$$

The corresponding central extension $\hat{\partial}$,

$$
0 \rightarrow \mathbb{C} \rightarrow \hat{\partial} \rightarrow \partial \rightarrow 0
$$

is called the Virasoro algebra. As a vector space, $\hat{\partial}=\partial \oplus \mathbb{C} \cdot \kappa$ with $\kappa$ central. The Lie bracket is determined by

$$
\left[d_{n}, d_{m}\right]=(n-m) d_{m+n}+\frac{1}{12}\left(n^{3}-n\right) \delta_{n,-m} \kappa .
$$

The action of $\partial$ on $\hat{\mathscr{G}}$ may be extended to an action of $\hat{\partial}$ by letting $\kappa$ act trivially. With this action, we form the semi-direct product

$$
m=\hat{\mathscr{G}} \beth \hat{\partial} .
$$

The Hermitian conjugation $x \mapsto x^{*}, x \in \hat{\mathscr{G}}$ is extended to $\hat{\partial}$ by setting

$$
\begin{aligned}
d_{n}^{*} & =d_{-n} ; \quad n \in \mathbb{Z}, \\
\kappa^{*} & =\kappa,
\end{aligned}
$$

and this extension is compatible with the action of $\hat{\partial}$ on $\hat{\mathscr{G}}$. Furthermore, the real subalgebra

$$
\partial_{k}=\left\{d \in \partial \mid d^{*}=-d\right\}
$$

coincides with real vector fields on $S^{1}$, by evaluating on $S^{1}$. Hence it is a subalgebra of the Lie algebra of Diff ${ }^{+}\left(S^{1}\right)$.

Finally, the extension

$$
\hat{\mathscr{G}}^{e}:=\hat{\mathscr{G}} \mathbb{C} \cdot d
$$

of $\hat{\mathscr{G}}$ by the degree derivation $d:=-d_{0}$,

$$
d x(n)=n x(n)
$$

will be convenient to describe the representation theory of $\hat{\mathscr{G}}$. 


\section{III.6. The Root Space Decomposition of $\hat{\mathscr{G}}^{e}$; Dominant Integral Weights}

The root space decomposition of $\hat{\mathscr{G}}^{e}$ relative to the abelian subalgebra $\hat{\mathfrak{h}}^{e}=$ $\mathfrak{h} \oplus \mathbb{C} \cdot c \oplus \mathbb{C} \cdot d$ can be described as follows. If $\lambda \in \mathfrak{h}^{*}$, extend $\lambda$ to $\hat{\mathfrak{h}}^{e}$ by setting $\lambda(c)=\lambda(d)=0$. Define $\delta \in\left(\hat{\mathfrak{h}}^{e}\right)^{*}$ by

$$
\delta(\mathfrak{h})=0, \quad \delta(c)=0, \quad \delta(d)=1 .
$$

Since $\left[h+d ; x_{\alpha}(n)\right]=(\alpha(h)+n) x_{\alpha}(n)$ for $\alpha \in \Delta, x_{\alpha} \in \mathscr{G}_{\alpha}$ and $h \in \mathfrak{h}$, it is clear that the roots of $\hat{\mathscr{G}}^{e}$ with respect to $\hat{\mathfrak{h}}^{e}$ are

$$
\hat{\Delta}=\{k \delta+\alpha \mid k \in \mathbb{Z}, \quad \alpha \in \Delta\} \cup\{k \delta \mid k \in \mathbb{Z}-\{0\}\}
$$

and the root spaces are

$$
\left(\hat{\mathscr{G}}^{e}\right)_{\alpha+n \delta}=\mathbb{C} \cdot e_{\alpha}(n), \quad\left(\hat{\mathscr{G}}^{e}\right)_{n \delta}=\{h(n) \mid h \in \mathfrak{h}\} .
$$

Set

$$
\hat{\Delta}_{+}=\{\alpha+n \delta \mid \alpha \in \Delta, n>0\} \cup\{n \delta \mid n>0\} \cup \Delta_{+},
$$

so that $\hat{\Delta}=\hat{\Delta}_{+} \cup\left(-\hat{\Delta}_{+}\right)$. The set of simple roots is $\hat{\pi}=\left\{\alpha_{0}, \alpha_{1}, \ldots \alpha_{l}\right\}$, where $\alpha_{1}, \ldots \alpha_{l}$ are the simple roots of $\mathscr{G}$ and $\alpha_{0}=\delta-\theta$. The corresponding coroots are

$$
\begin{aligned}
& \hat{h}_{\alpha_{0}}=c-h_{\theta}, \\
& \hat{h}_{\alpha_{i}}=h_{\alpha_{\imath}}, \quad i=1, \ldots l .
\end{aligned}
$$

Define the fundamental weights $\hat{\Lambda}_{0}, \ldots \hat{\Lambda}_{l} \in\left(\hat{\mathfrak{h}}^{e}\right)^{*}$ by

$$
\begin{aligned}
& \hat{\Lambda}_{i}\left(\hat{h}_{\alpha_{\jmath}}\right)=\delta_{\imath j}, \quad i, j=0, \ldots l, \\
& \hat{\Lambda}_{i}(d)=0 .
\end{aligned}
$$

Note that the restriction of $\hat{\Lambda}_{1}, \ldots \hat{\Lambda}_{l}$ to $\mathfrak{h}$ are the fundamental weights for $\mathscr{G}$ and we have

$$
\hat{\Lambda}_{i}=\Lambda_{i}+a_{\imath} \hat{\Lambda}_{0} \quad i=1, \ldots l .
$$

Put $\varrho:=\sum_{\imath=0}^{l} \hat{\Lambda}_{\imath}$; then $\hat{\varrho}=\varrho+g \hat{\Lambda}_{0}$.

Let $P:=\sum_{i=0}^{l} \mathbb{Z} \cdot \hat{\Lambda}_{i}$ be the weight lattice of $\hat{\mathscr{G}}^{e}$ and $P_{+}:=\sum_{i=0}^{l} \mathbb{Z}_{+} \cdot \hat{\Lambda}_{i}$ be the set of dominant integral weights. Given $\hat{\Lambda} \in P_{+}$, the positive number $\hat{\Lambda}(c)$ is called the level of $\hat{\Lambda}$. Note that

$$
\hat{\Lambda}_{i}(c)=a_{i}, \quad i=1, \ldots l .
$$

Given $m \in \mathbb{Z}_{+}$, denote by $P_{+}^{(m)}$ the set of $\hat{\Lambda} \in P_{+}$of level $m$. This is a finite set for each $m \in \mathbb{Z}_{+}$and $P_{+}^{(0)}=0$.

We extend the bilinear form $(\cdot \mid \cdot)$ on $\mathfrak{h}^{*}$ to a symmetric bilinear form on $\left(\hat{\mathfrak{h}}^{e}\right)^{*}$ by

$$
\left(\mathfrak{h}^{*} \mid \mathbb{C} \delta+\mathbb{C} \hat{\Lambda}_{0}\right)=0, \quad(\delta \mid \delta)=\left(\hat{\Lambda}_{0} \mid \hat{\Lambda}_{0}\right)=0, \quad\left(\delta \mid \hat{\Lambda}_{0}\right)=1 .
$$

Notice that $\hat{\Lambda}(c)=(\hat{\Lambda} \mid \delta)$. 
We will also need the following triangular decomposition of $\hat{\mathscr{G}}^{e}$ :

$$
\hat{\mathscr{G}}^{e}=\hat{n}_{+} \oplus \hat{\mathfrak{h}}^{e} \oplus \hat{n}_{-},
$$

where

$$
\begin{aligned}
& \hat{n}_{+}:=\left(\sum_{\alpha \in \Delta_{+}} \oplus \mathscr{G}_{\alpha} \otimes 1\right) \oplus \mathscr{G} \otimes \mathbb{C}[t], \\
& \hat{n}_{-}:=\left(\sum_{-\alpha \in \Delta_{+}} \oplus \mathscr{G}_{\alpha} \otimes 1\right) \oplus \mathscr{G} \otimes \mathbb{C}\left[t^{-1}\right] .
\end{aligned}
$$

\section{III.7. Properties of Irreducible Integrable Highest Weight Modules of $\hat{\mathscr{G}}^{e}$}

Let $\hat{\Lambda} \in P_{+}$be a dominant integral weight of $\hat{\mathscr{G}}^{e}$. A $\hat{\mathscr{G}}^{e}$-module $(V ; \pi)$ is called a highest weight module with highest weight $\hat{\Lambda}$, if there exists a vector $v_{\hat{\Lambda}} \in V$ such that

$$
\pi\left(\hat{n}_{+}\right) \cdot v_{\hat{\Lambda}}=0, \quad \pi(h) \cdot v_{\hat{\Lambda}}=\hat{\Lambda}(h) \cdot v_{\hat{\Lambda}} \quad \text { for } h \in \hat{\mathfrak{h}}^{e},
$$

and

$$
\pi\left(U\left(\hat{\mathscr{G}}^{e}\right)\right) \cdot v_{\hat{\Lambda}}=V,
$$

where $U\left(\hat{\mathscr{G}}^{e}\right)$ denotes the enveloping algebra of $\hat{\mathscr{G}}^{e}$. There exists, up to isomorphism, a unique irreducible highest weight module $\left(L(\hat{\Lambda}), \pi_{\hat{\Lambda}}\right)$ with highest weight $\hat{\Lambda} \in P_{+}$.

The module $L(\hat{\Lambda})$ has the following properties [47, 61].

(i) There exists on $L(\hat{\Lambda})$ a positive definite Hermitian form $\langle\cdot \mid \cdot\rangle$ such that

$$
\left\langle\pi_{\hat{\Lambda}}(x) u \mid v\right\rangle=\left\langle u \mid \pi_{\hat{\Lambda}}\left(x^{*}\right) v\right\rangle, \quad \forall u, v \in L(\hat{\Lambda}), x \in \hat{\mathscr{G}}^{e} .
$$

Such a Hermitian form is said to be contravariant with respect to the ${ }^{*}$-conjugation of $\hat{\mathscr{G}}^{e}$. In particular, it follows from contravariance that the elements of $\hat{\mathscr{G}}_{k}$ are represented by skew-Hermitian operators on $L(\hat{\Lambda})$.

(ii) The action of $\hat{\mathscr{G}}$ on $L(\hat{\Lambda})$ can be extended to the semidirect product of $\hat{\mathscr{G}}$ and $\hat{\partial}$ by the Sugawara construction:

$$
\pi_{\hat{\Lambda}}\left(d_{n}\right):=L_{n}:=\frac{1}{2(m+g)} \sum_{j \in \mathbb{Z}} \sum_{i}: u_{\imath}(-j) u^{i}(j+n):,
$$

where $\left\{u_{i}\right\}$ and $\left\{u^{\jmath}\right\}$ are dual bases of $\mathscr{G}, m$ is the level of $\hat{\Lambda}, g$ is the dual Coxeter number of $\mathscr{G}$ and the normal ordering symbol : $u(s) v(r):$ stands for $u(s) v(r)$ if $s \leq r$ and for $v(r) u(s)$ if $s>r$. The Hermitian form $\langle\cdot \mid \cdot\rangle$ is contravariant for the extended representation and the central element $\kappa$ of $\hat{\partial}$ is represented by

$$
\pi_{\hat{\Lambda}}(\kappa)=\sigma \cdot I, \quad \sigma=\frac{m \operatorname{dim} \mathscr{G}}{(m+g)} .
$$

The constant $\sigma$ is called the conformal anomaly. Furthermore,

$$
L_{0}=h_{\hat{\Lambda}} \cdot I-\pi_{\hat{\Lambda}}(d), \quad h_{\hat{\Lambda}}:=\frac{(\hat{\Lambda}+2 \hat{\varrho} \mid \hat{\Lambda})}{2(m+g)},
$$

so that the eigenvalues of $L_{0}$ on $L(\hat{\Lambda})$ are given by $h_{\hat{\Lambda}}+\mathbb{Z}_{+}$. The constant $h_{\hat{\Lambda}}$ is called the trace anomaly. 
(iii) Denote by $\mathscr{H}_{\hat{\Lambda}}:=\overline{L(\hat{\Lambda})}$ the Hilbert space completion of $L(\hat{\Lambda})$ with respect to the hermitian form $\langle\cdot \mid \cdot\rangle$. The representation $\pi_{\hat{\Lambda}}$ restricted to $\hat{\mathscr{G}}_{k}$ integrates to a strongly continuous, irreducible, unitary representation of $\widehat{L G}_{0}$ on $\mathscr{H}_{\hat{\Lambda}}$. Furthermore the representation $\pi_{\hat{\Lambda}}$ restricted to $\partial_{k}$ integrates to a strongly continuous, projective representation of $\operatorname{Diff}^{+}\left(S^{1}\right)$ on $\mathscr{H}$ which implements the action of $\operatorname{Diff}^{+}\left(S^{1}\right)$ on $\widehat{L G}_{0}$. (iv) The operator $L_{0}$ is the generator of rotations of the circle on $\mathscr{H}_{\hat{\Lambda}}, e^{-\beta L_{0}}$ is trace class for $\beta>0$ and satisfies the following asymptotic estimate [47,62] as $\beta \rightarrow 0$, $\beta>0$ :

$$
\operatorname{tr}_{\mathscr{H}_{\hat{\Lambda}}} e^{-\beta L_{0}} \sim a(\hat{\Lambda}) \exp (-\beta \sigma / 24) \exp \left(\pi^{2} \sigma / 6 \beta\right),
$$

where $a(\hat{\Lambda})$ is a constant depending only on $\hat{\Lambda} \in P_{+}$.

\section{III.8. Vacuum Representations of $\widehat{L G}_{0}$; the Basic Representation}

Let $\hat{\Lambda} \in P_{+}, \Lambda$ be the restriction of $\hat{\Lambda}$ to $\mathfrak{h}$ and $\left(\pi_{\hat{\Lambda}} ; \mathscr{H}_{\hat{\Lambda}}\right)$ the corresponding representation of $\widehat{L G}_{0}$ obtained in Sect. III.7. These representations form a complete list of irreducible positive-energy representations of $\widehat{L G}_{0}$.

An irreducible vacuum representation of $\widehat{L G}_{0}$ is an irreducible, positive-energy representation having a unique lowest eigenvalue vector for $L_{0}$ invariant under the whole Moebius subgroup of $\operatorname{Diff}^{+}\left(S^{1}\right)$. Such representations are easily determined: notice that $G_{0}$ can be identified with the subgroup of constant loops in $L G_{0}$. Constant loops are invariant under rigid rotations of the circle $S^{1}$ and consequently the unitary representation $\pi_{\hat{\Lambda}}$ of $G_{0}$ on $\mathscr{H}_{\hat{\Lambda}}$ is reduced by the eigenspaces of $L_{0}$ [58]. In particular, the lowest eigenspace of $L_{0}$ (i.e., the eigenspace to the eigenvalue $h_{\hat{\Lambda}}$ ) carries the irreducible representation of $G_{0}$ corresponding to the dominant weight $\Lambda$ on $\mathfrak{h}$. This implies that $L_{0}$ has a unique lowest eigenvalue vector if and only if $\Lambda \equiv 0$ on $\mathfrak{h}$, that is, if and only if $\hat{\Lambda}=m \hat{\Lambda}_{0}, h_{\hat{\Lambda}}=0$. One checks easily that $v_{\hat{\Lambda}}$ is annihilated by $L_{ \pm 1}$ in this case, that is $v_{\hat{\Lambda}}$ is invariant under the Moebius subgroup of $\operatorname{Diff}^{+}\left(S^{1}\right)$. Hence, for each level, there is a unique irreducible vacuum representation of $\widehat{L G}_{0}$, determined by the dominant integral weight $m \hat{\Lambda}_{0}, m \in \mathbb{Z}_{+}$.

The level one vacuum representation is called the basic representation of $\widehat{L G}_{0}$. If $G_{0}$ is simply connected and simply laced, every irreducible positive-energy representation of $\widehat{L G}_{0}$ can be obtained from the basic representation as follows (see [42], Theorem 9.3.9).

Let $Z$ be the center of $G_{0}$ and $g \in Z$. Pick any smooth path $\alpha_{g}: \mathbb{R} \rightarrow G_{0}$ such that $\alpha(0)=e, \alpha(2 \pi)=g, \alpha(\theta+2 \pi)=g \cdot \alpha(\theta)$. If, $\eta \in L G_{0}$, we denote by $\eta_{\alpha_{g}}$ the loop obtained by conjugating with $\alpha_{g}$; the map $\eta \rightarrow \eta_{\alpha_{g}}$ defines an outer automorphism of $L G_{0}$. The set of representations $\alpha_{g}^{*} \pi_{0}$ obtained by composing $\pi_{0}$ with such automorphisms are precisely the irreducible, level-one representations of $\widehat{L G}_{0}$.

Furthermore, any representation of level $m$ is a direct summand of $i_{m}^{*} \pi_{1}$, where $\pi_{1}$ is a level one representation of $\widehat{L G}_{0}, i_{m}: S^{1} \rightarrow S^{1}$ is any map of degree $m$ and $i_{m}^{*} \pi_{1}$ denotes the representation obtained by composing $\pi_{1}$ with $i_{m}$. 
III.9. Local Algebras in Irreducible Representation of $\widehat{L G}_{0}$

Let $\hat{\Lambda} \in P_{+}$and $\left(\pi_{\hat{\Lambda}} ; \mathscr{H}_{\hat{\Lambda}}\right)$ be the corresponding irreducible representation of $\widehat{L G}_{0}$. Define a local net $\left\{\mathscr{C}_{\hat{\Lambda}}(I)\right\}_{I \subset S^{1}}$ on $\mathscr{H}_{\hat{\Lambda}}$ by

$$
\mathscr{A}_{\hat{\Lambda}}(I):=\left\{\pi_{\hat{\Lambda}}(\hat{\mathscr{C}}(I))\right\}^{\prime \prime} .
$$

The following result is an easy consequence of the preceding discussion.

Theorem 3.2. (i) For ever $\hat{\Lambda} \in P_{+}$, the collection $\left\{\mathscr{A}_{\hat{\Lambda}}(I)\right\}_{I \subset S^{1}}$ is a conformal net on $\mathscr{H}_{\hat{\Lambda}}$.

(ii) Let $\hat{\Lambda}=m \hat{\Lambda}_{0}, m \in \mathbb{Z}_{+}$. The net $\left\{\mathscr{A}_{\hat{\Lambda}}(I)\right\}_{I \subset S^{1}}$ is the net of the vacuum sector of a conformal field theory in the sense of Chap. II. In particular, this net satisfies duality, and the local algebras $\mathscr{A}_{\hat{\Lambda}}(I)$ are hyperfinite type $I I I_{1}$ factors.

Proof. Ad (i). $\mathscr{C}_{\hat{\Lambda}}(I)$ is the weak closure of $\pi_{\hat{\Lambda}}(\hat{\mathscr{C}}(I))$, so that isotony and locality follow from the corresponding properties of the groups $\hat{\mathscr{b}}(I)$ derived in Sect. III.4 (see Lemma 3.1).

From Sect. III.7 (iii), we know that $\mathscr{H}_{\hat{\Lambda}}$ carries as strongly continuous, projective representation of the Moebius group under which $\pi_{\hat{\Lambda}}(\hat{\mathscr{B}}(I))$ transforms covariantly for every $I$, so that covariance of the net follows.

Ad (ii). We know from Sect. III. 8 that $\mathscr{H}_{\hat{\Lambda}}$, with $\hat{\Lambda}=m \hat{\Lambda}_{0}$, has a unique vector invariant under the Moebius group and that the spectrum of $L_{0}$ is positive on $\mathscr{H}_{\hat{\Lambda}}$. The vacuum vector $v_{\hat{\Lambda}}$ of $\mathscr{H}_{\hat{\Lambda}}$ is by definition cyclic for the von Neumann algebra $\mathfrak{A}=\left\{\bigcup_{I \subset S^{1}} \mathscr{C}_{\hat{\Lambda}}(I)\right\}^{\prime \prime}$. Hence all properties of Definition 2.5 are fulfilled. Furthermore, the asymptotic behaviour of $\operatorname{tr}_{\mathscr{H}_{\hat{\Lambda}}} e^{-\beta L_{0}}$ given in Sect. III.7 (iv) implies that Theorem 2.13 is applicable. Haag duality follows from Theorem 2.19. This completes the proof of the theorem.

As a matter of fact, the theorem of Pressley and Segal presented in Sect. III.8 (see ref. [42], Theorem 9.3.9) implies that local algebras are hyperfinite type $I I I_{1}$ factors in any irreducible positive-energy representation of $\widehat{L G}_{0}$.

Theorem 3.3. For every $\hat{\Lambda} \in P_{+}$and $I \subset S^{1}, \mathscr{A}_{\hat{\Lambda}}(I)$ is isomorphic to the hyperfinite type $I I I_{1}$ factor.

Proof. If $\hat{\Lambda}$ is a level one dominant integral weight, then $\pi_{\hat{\Lambda}} \cong \alpha_{g}^{*} \pi_{0}$, where $\alpha_{g}$ is a smooth path from $e$ to $g \in Z$ along $S^{1}$. Choose $\alpha_{g}$ in such a way that $\left.\alpha_{g}\right|_{I} \equiv e$. It then follows that $\pi_{\hat{\Lambda}_{0}}$ and $\alpha_{g}^{*} \pi_{\hat{\Lambda}_{0}}$ coincide on $\hat{\mathscr{A}}(I)$. Hence $\mathscr{A}_{\hat{\Lambda}}(I)=\mathscr{A}_{\hat{\Lambda}_{0}}(I)$, and, since all local algebras are unitarily equivalent, by Moebius covariance, this completes the proof in the level one case.

Next, let $\pi_{\hat{\Lambda}}$ be a level $m$ representation. We know that $\pi_{\hat{\Lambda}}$ is a direct summand of $i_{m}^{*} \pi_{1}$, where $\pi_{1}$ is a level one representation and $i_{m}: S^{1} \rightarrow S^{1}$ has degree $m$. Let $E \in i_{m}^{*} \pi_{1}\left(\widehat{L G}_{0}\right)^{\prime}$ be the projection on $\pi_{\hat{\Lambda}}$. Then $\mathscr{C}_{\hat{\Lambda}}(I)=\left\{i_{m}^{*} \pi_{1}(\hat{\mathscr{b}}(I))\right\}_{E}^{\prime \prime}$ (see [63], Chapt. I, $\S 2$, Prop. 1). Choose $i_{m}$ such that $\left.i_{m}\right|_{I} \equiv$ id $\left.\right|_{I}$ so that $\left\{i_{m}^{*} \pi_{1}(\hat{\mathscr{b}}(I))\right\}^{\prime \prime}=\left\{\pi_{1}(\hat{\mathscr{b}}(I))\right\}^{\prime \prime}$ is the hyperfinite type $I I I_{1}$ factor, since $\pi_{1}$ is of level one. The projection $E$ belongs to $\left\{i_{m}^{*} \pi_{1}(\hat{\mathscr{b}}(I))\right\}^{\prime}$, that is to $\left\{\pi_{1}(\hat{\mathscr{b}}(I))\right\}^{\prime}$. Since $\pi_{1}(\hat{\mathscr{C}}(I))^{\prime}$ is a factor, the central support of $E$ is $\mathbb{1}$ and the induction of $\left\{\pi_{1}(\hat{\mathscr{C}}(I))\right\}^{\prime \prime}$ 
on $\left\{\pi_{1}(\hat{\mathscr{C}}(I))\right\}_{E}^{\prime \prime}$ is an isomorphism (see [63], Chapt. I, $\S 2$, Prop. 2) and the theorem is proved.

This completes our discussion of the properties of conformal nets associated to positive-energy representations of loop groups. The corresponding algebraic quantum field theories and their superselection structure will be discussed in next chapter.

For the sake of completeness, we state without further details the analogue of Theorem 3.2 for representations of the Virasoro algebra.

Theorem 3.4. Let $L((h ; c))$ be an irreducible unitary positive-energy vacuum representation of the Virasoro algebra, that is,

(i) $c=1-\frac{6}{m(m+1)}, m=3,4, \ldots, h=0$, or

(ii) $c \geq 1, \quad h=0$.

Then the local algebras constructed from the corresponding projective representation of $\operatorname{Diff}^{+}\left(S^{1}\right)$ are hyperfinite type $I I I_{1}$ factors, and the net determined by these local algebras is dual.

The proof of this theorem (which is very similar to the one of Theorem 3.2 given in this chapter) relies on results of Goodman and Wallach [78] on the integrability of positive-energy unitary representations of the Virasoro algebra and from explicit formulas for the characters of these representations [79].

\section{Representation Theory of Conformal Nets}

\section{IV.1 Definitions, Basic Properties}

Let $\left\{\mathscr{H} ; \pi_{0} ; \mathscr{b} ; \Omega\right\}$ be the vacuum sector of a conformal field theory fulfilling all the properties specified in Chap. II.

In this chapter we use the following conventions:

- $\widetilde{P S U}(1 ; 1)$ denotes the universal covering group of the Moebius group $\operatorname{PSU}(1 ; 1)$.

- Elements of $\widetilde{P S U}(1 ; 1)$ are written as small latin letters with tildes: $\tilde{g}, \tilde{h}, \tilde{g}_{1}$, etc... If $p: \widetilde{P S U}(1 ; 1) \rightarrow P S U(1 ; 1)$ is the canonical projection then the image $p(\tilde{g}) \in P S U(1 ; 1)$ of $\tilde{g} \in \widetilde{P S U}(1 ; 1)$ is denoted by the same letter, without tilde: $p(\tilde{g})=g$.

- We denote by $\pi_{0}^{M}$ (instead of $\pi_{0}$ ) the unitary representation of the Moebius group on the vacuum sector. By $\pi^{M}$ we denote other projective, unitary representations of the Moebius group (i.e., unitary representations of $\widetilde{P S U}(1 ; 1)$ ).

- We define, for $g \in P S U(1 ; 1)$,

$$
\begin{aligned}
\alpha_{g}(A) & :=\operatorname{ad} \pi_{0}^{M}(g)(A) \\
& =\pi_{0}^{M}(g) A \pi_{0}^{M}(g)^{*}, \quad \forall A \in \mathscr{B}(\mathscr{H}) .
\end{aligned}
$$

The conformal net $\mathscr{C}_{0}:=\{A(I)\}_{I \subset S^{1}}$ constructed in Chap. II is a representation independent object characterized by

(i) the fact that each local algebra $\mathscr{\ell}(I)$ is isomorphic to the unique hyperfinite type $I I I_{1}$ factor;

(ii) the inclusions $\mathscr{A}(I) \subseteq \mathscr{A}(J)$ whenever $I \subseteq J$, for open, non-dense intervals of $S^{1} ; A(I) \subseteq \mathscr{A}(J)^{\prime}$ if $I \subseteq J^{\prime}$; 
(iii) Moebius covariance: for $g \in P S U(1 ; 1), \alpha_{g}$ defines an automorphism of the net $\mathscr{b}_{0}$, i.e.,

$$
\alpha_{g}(\mathscr{b}(I))=\mathscr{b}(g \cdot I)
$$

holds for all intervals $I \subset S^{1}$ and all $g \in P S U(1 ; 1)$.

This chapter is devoted to the study of the representation theory of conformal nets.

Definition 4.1. A representation $\pi$ of the conformal net $\mathscr{A}_{0}$ on the separable Hilbert space $\mathscr{H}_{\pi}$ is a consistent family $\pi=\left\{\pi_{I}\right\}_{I \subset S^{1}}$ of representations of the local algebras $\{\mathscr{C}(I)\}_{I \subset S^{1}}$ which is Moebius covariant and satisfies the spectrum condition, that is,

(i) if $I \subseteq J$ then $\left.\pi_{J}\right|_{\mathscr{C}(I)}=\pi_{I}$ (consistence).

(ii) There exists a unitary representation $\pi^{M}$ of $\widetilde{P S U}(1 ; 1)$ on $\mathscr{H}_{\pi}$ (i.e., a projective representation of $P S U(1 ; 1)$ ) such that

$$
\pi^{M}(\tilde{g}) \pi_{I}(A) \pi^{M}(\tilde{g})^{*}=\pi_{g \cdot I}\left(\alpha_{g}(A)\right)
$$

holds, for all $A \in \mathscr{A}(I), \tilde{g} \in \widetilde{P S U}(1 ; 1)$ (Moebius covariance).

(iii) The spectrum of the infinitesimal generator of rotations on $\mathscr{H}_{\pi}$ is positive (spectrum condition).

Such representations always respect the local structure of $\mathscr{A}_{0}$.

Lemma 4.2. Let $\pi$ be a representation of $\mathscr{b}_{0}$. If $I \subseteq J^{\prime}$, then

$$
\pi_{I}(\mathscr{A}(I)) \subseteq \pi_{J}(\mathscr{A}(J))^{\prime} .
$$

Proof. Let $I \Subset J^{\prime}$. Then there exists an interval $K \supseteq I \cup J$ such that, by consistence, we have, for $A \in \mathscr{A}(I)$ and $B \in \mathscr{A}(J)$,

$$
\begin{aligned}
\pi_{I}(A) \pi_{J}(B) & =\pi_{K}(A) \pi_{K}(B) \\
& =\pi_{K}(A B)=\pi_{K}(B A) \\
& =\pi_{K}(B) \pi_{K}(A)=\pi_{J}(B) \pi_{I}(A) .
\end{aligned}
$$

Hence $\pi_{I}(\mathscr{A}(I)) \subseteq \pi_{J}(\mathscr{A}(J))^{\prime}$, for all $I \Subset J^{\prime}$. But because of Moebius covariance [31],

$$
\pi_{J^{\prime}}\left(\mathscr{A}\left(J^{\prime}\right)\right)=\bigvee_{I \Subset J^{\prime}} \pi_{I}(\mathscr{A}(I)) \subseteq \pi_{J}(\mathscr{A}(J))^{\prime}
$$

and this completes the proof of the lemma.

Definition 4.3. (i) A representation $\pi$ of $\mathscr{A}_{0}$ is irreducible if the von Neumann algebra

$$
\pi\left(\mathscr{A}_{0}\right)^{\prime}:=\left\{\pi_{I}(\mathscr{A}(I)), \quad I \subset S^{1}\right\}^{\prime}
$$

is equal to $\mathbb{C} \cdot \mathbb{1}_{\mathscr{H}_{\pi}}$; otherwise $\pi$ is reducible.

(ii) Two representations $\pi$ and $\tilde{\pi}$ are unitarily equivalent if there exists a unitary operator $U: \mathscr{H}_{\pi} \rightarrow \mathscr{H}_{\tilde{\pi}}$ such that

$$
\tilde{\pi}_{I}(\cdot) U=U \pi_{I}(\cdot) \quad \forall I \subset S^{1} .
$$

(iii) An equivalence class of representations of the conformal net $\mathscr{b}_{0}$ is called a sector. If $\pi$ is a representation of $\mathscr{A}_{0}$, we denote the corresponding sector by $[\pi]$.

The following observation was first made by Buchholz, Mack and Todorov [30]. 
Lemma 4.4. Any representation $\pi$ of $\mathscr{b}_{0}$ is locally unitarily equivalent to the identity representation, that is, for each interval $I \subset S^{1}$,

$$
\left.\left.\pi_{I}\right|_{2(I)} \cong \mathrm{id}\right|_{2(I)}
$$

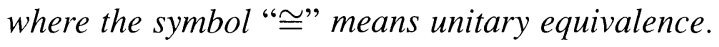

In our case, this lemma follows from the fact that the local algebras are hyperfinite type $I I I_{1}$ factors. This motivates the following definition.

Definition 4.5. A representation $\rho=\left\{\rho_{I}\right\}_{I \subset S^{1}}$ of the conformal net $\mathscr{t}_{0}$ on the vacuum Hilbert space $\mathscr{H}$ is said to be localized in the interval $I_{0}$ if on the complement, $I_{0}^{\prime}$, of $I_{0}$

$$
\varrho_{I_{0}^{\prime}}=\mathrm{id}_{I_{0}^{\prime}}
$$

holds.

As an immediate consequence of Lemma 4.4 we obtain the following result.

Lemma 4.6. If $[\pi]$ is a sector of representations of. $A_{0}$, then, for each interval $I_{0} \subset S^{1}$, there exists a representation $\rho \in[\pi]$ localized in $I_{0}$.

Proof. Since, by Lemma $4.4, \pi_{I_{0}^{\prime}} \cong \mathrm{id}_{I_{0}^{\prime}}$, we can choose a bijective isometry $U: \mathscr{H} \rightarrow \mathscr{H}_{\pi}$ such that

$$
\pi_{I_{0}^{\prime}}(A) U=U A \quad \forall A \in \mathscr{\ell}\left(I_{0}^{\prime}\right)
$$

and define $\rho_{I}(\cdot):=U^{*} \pi_{I}(\cdot) U, \forall I \subset S^{1}$. Clearly, $\rho$ is a representation in $[\pi]$ localized in $I_{0}$, by construction.

Let $\rho_{1}, \rho_{2} \in[\pi]$ be localized in $I_{1}, I_{2}$ respectively. If $I$ is an interval containing $I_{1}$ and $I_{2}$, then duality and the localization properties of $\rho_{1}, \rho_{2}$ imply that any unitary operator $\Gamma_{\rho_{1} \rho_{2}}$ intertwining $\rho_{1}$ and $\rho_{2}$,

$$
\rho_{1, J}(A) \Gamma_{\rho_{1} \rho_{2}}=\Gamma_{\rho_{1} \rho_{2}} \rho_{2, J}(A), \quad \forall J \subset S^{1}, \quad A \in A_{\text {A }}(J)
$$

belongs to $\ell(I)$. Explicit examples of such operators are the cocycles of the Moebius group constructed below. For notational convenience, we will also write the preceding equation in compact form,

$$
\rho_{1}(A) \Gamma_{\rho_{1} \rho_{2}}=\Gamma_{\rho_{1} \rho_{2}} \rho_{2}(A), \quad \forall A \in \mathscr{\ell}_{0} .
$$

That is, if an equation holds for all intervals $J \subset S^{1}$, we suppress the interval indexing a collection of representations $\rho=\left\{\rho_{I}\right\}_{I \subset S^{1}}$.

If $\rho$ is a representation of $\mathscr{b}_{0}$ localized in $I_{0}$, we may define shifted representations $\rho_{g}$ localized in $g \cdot I_{0}$ by

$$
\rho_{g, I}(A):=\alpha_{g} \circ \rho_{g^{-1} \cdot I} \circ \alpha_{g^{-1}}(A), \quad \forall A \in \mathscr{A}_{0}(I) .
$$

It follows from Moebius covariance that $\rho$ and $\rho_{g}$ are unitarily equivalent for all $g \in P S U(1 ; 1)$ :

$$
\rho_{g}(A)=\pi_{0}^{M}(g) \pi_{\rho}^{M}\left(\tilde{g}^{-1}\right) \rho(A) \pi_{\rho}^{M}\left(\tilde{g}^{-1}\right)^{*} \pi_{0}^{M}(g)^{*}, \quad \forall A \in \mathscr{A}_{0},
$$


where $\pi_{\rho}^{M}$ is the representation of the Moebius group implementing covariance of the representation $\rho$ (Definition 4.1 (ii)). Setting $\Gamma_{\rho}(\tilde{g}):=\pi_{0}^{M}(g) \pi_{\rho}^{M}\left(\tilde{g}^{-1}\right)$, we may rewrite the last equation as

$$
\rho_{g}(A) \Gamma_{\rho}(\tilde{g})=\Gamma_{\rho}(\tilde{g}) \rho(A), \quad \forall A \in \mathscr{A}_{0}, \quad \tilde{g} \in \widetilde{P S U}(1 ; 1)
$$

and the intertwiners $\Gamma_{\rho}(\tilde{g}), \tilde{g} \in \widetilde{P S U}(1 ; 1)$ satisfy the cocycle identity,

$$
\Gamma_{\rho}\left(\tilde{g}_{1} \cdot \tilde{g}_{2}\right)=\alpha_{g_{1}}\left(\Gamma_{\rho}\left(\tilde{g}_{2}\right)\right) \Gamma_{\rho}\left(\tilde{g}_{1}\right), \quad \forall \tilde{g}_{1}, \tilde{g}_{2} \in \widetilde{P S U}(1 ; 1) .
$$

Conversly, given a cocycle $\Gamma_{\rho}(\tilde{g}), \tilde{g} \in \widetilde{P S U}(1 ; 1)$ of the Moebius group which satisfies Eq. (4.1), one can reconstruct a unitary representation of $\widetilde{P S U}(1 ; 1)$ which implements the automorphisms $\alpha_{g}$ of $\mathscr{A}_{0}$ in the representation $\rho$ (see [14]).

Note that by our definition,

$$
\left(\rho_{g_{1}}\right)_{g_{2}}=\alpha_{g_{2}} \circ \rho_{g_{1}} \circ \alpha_{g_{2}^{-1}}=\alpha_{g_{2} g_{1}} \circ \rho \circ \alpha_{\left(g_{2} g_{1}\right)^{-1}}=\rho_{g_{2} g_{1}} .
$$

If $\Gamma_{\rho}(\tilde{g})$ is the cocycle for $\rho$, then applying $\alpha_{g}^{\prime}$ on both sides of Eq. (4.1) we obtain

$$
\left(\rho_{g}\right)_{g^{\prime}}(A) \alpha_{g^{\prime}}\left(\Gamma_{\rho}(\tilde{g})\right)=\alpha_{g^{\prime}}\left(\Gamma_{\rho}(\tilde{g})\right) \rho_{g^{\prime}}(A), \quad \forall A \in \mathscr{C}_{0} .
$$

Hence the cocycle identity is consistent with

$$
\rho_{g_{1} g_{2}}(A) \Gamma_{\rho}\left(\tilde{g}_{1} \cdot \tilde{g}_{2}\right)=\Gamma_{\rho}\left(\tilde{g}_{1} \cdot \tilde{g}_{2}\right) \rho(A), \quad \forall A \in \mathscr{\ell}_{0} .
$$

Namely, $\rho_{g_{1} g_{2}}(A)=\left(\rho_{g_{2}}\right)_{g_{1}}(A)$ and $\Gamma_{\rho}\left(\tilde{g}_{1} \cdot \tilde{g}_{2}\right)=\alpha_{g_{1}}\left(\Gamma_{\rho}\left(\tilde{g}_{2}\right)\right) \Gamma_{\rho}\left(\tilde{g}_{1}\right)$ so that

$$
\begin{aligned}
\rho_{g_{1} \cdot g_{2}}(A) \Gamma_{\rho}\left(\tilde{g}_{1} \cdot \tilde{g}_{2}\right) & =\left(\rho_{g_{2}}\right)_{g_{1}}(A) \alpha_{g_{1}}\left(\Gamma_{\rho}\left(\tilde{g}_{2}\right)\right) \Gamma_{\rho}\left(\tilde{g}_{1}\right) \\
& =\alpha_{g_{1}}\left(\Gamma_{\rho}\left(\tilde{g}_{2}\right)\right) \rho_{g_{1}}(A) \Gamma_{\rho}\left(\tilde{g}_{1}\right) \\
& =\alpha_{g_{1}}\left(\Gamma_{\rho}\left(\tilde{g}_{2}\right)\right) \Gamma_{\rho}\left(\tilde{g}_{1}\right) \rho(A) \\
& =\Gamma_{\rho}\left(\tilde{g}_{1} \cdot \tilde{g}_{2}\right) \rho(A) .
\end{aligned}
$$

Finally, we remark that if $\rho$ is localized in $I_{0}$, then for any interval $J \supseteq I_{0}$ one has, by duality and locality, that

$$
\rho_{J}(\mathscr{C}(J)) \subseteq \mathscr{C}(J)
$$

so that $\rho_{J}$ defines an endomorphism of $\mathscr{C}(J)$. In particular, the pair $\left\{\rho_{J}(\mathscr{A}(J))\right.$; $\mathscr{A}(J)\}$ defines an inclusion of hyperfinite type $I I I_{1}$ factors.

\section{IV.2. Composition of Sectors}

Given two sectors $\left[\pi_{1}\right]$ and $\left[\pi_{2}\right]$ of $\mathscr{A}_{0}$, we define a composed sector $\left[\pi_{1} \hat{o} \pi_{2}\right]$ by picking two representations $\rho_{1} \in\left[\pi_{1}\right], \rho_{2} \in\left[\pi_{2}\right]$ localized in some common interval $I_{0}$ and defining a composed representation $\rho_{1} \hat{o} \rho_{2}$ of $\mathscr{t}_{0}$. There are several possible ways of defining composition in the present situation $[15,20,25,32]$. We choose the alternative which is closest in spirit to reference [20].

We define the representation $\rho_{1} \hat{o} \rho_{2}$ of $\mathscr{C}_{0}$ by specifying $\left(\rho_{1} \hat{o} \rho_{2}\right)_{I}$ for each interval $I \subset S^{1}$. Let $I$ be such that there exists $J \subset S^{1}$ with $I \cup I_{0} \subseteq J$. Then $\rho_{1, J}, \rho_{2, J}$ define endomorphisms of $\mathscr{C}(J) \supseteq \mathscr{\ell}(I)$ so that they can be composed. We set

$$
\left(\rho_{1} \hat{\circ} \rho_{2}\right)_{I}:=\left.\rho_{1, J} \circ \rho_{2, J}\right|_{.,(I)} \text {. }
$$


If $I$ is such that $\left(I \cup I_{0}\right)^{-}$covers $S^{1}$, we choose $\tilde{I}_{0} \subseteq I_{0}$ such that $\tilde{I}_{0} \cup I \subseteq J$ for some interval $J \subset S^{1}$. There exist two representations $\tilde{\rho}_{1}, \tilde{\rho}_{2}$ localized in $\tilde{I}_{0}$ unitarily equivalent to $\rho_{1}, \rho_{2}$ and intertwiners $\Gamma_{\rho_{1} \tilde{\rho}_{1}}, \Gamma_{\rho_{2} \tilde{\rho}_{2}} \in \mathscr{A}\left(I_{0}\right)$ such that

$$
\rho_{i}(\cdot)=\Gamma_{\rho_{\imath} \tilde{\rho}_{\imath}} \tilde{\rho}_{\imath}(\cdot) \Gamma_{\rho_{\imath} \tilde{\rho}_{i}}^{*}, \quad i=1,2 .
$$

We define

$$
\left(\rho_{1} \hat{\circ} \rho_{2}\right)_{I}(\cdot):=\Gamma_{\rho_{1} \tilde{\rho}_{1}} \tilde{\rho}_{1, I_{0}}\left(\Gamma_{\rho_{2} \tilde{\rho_{2}}}\right) \tilde{\rho}_{1, J} \circ \tilde{\rho}_{2, J}(\cdot) \tilde{\rho}_{1, I_{0}}\left(\Gamma_{\rho_{2} \tilde{\rho}_{2}}\right)^{*} \Gamma_{\rho_{1} \tilde{\rho}_{1}}^{*} \cdot
$$

Lemma 4.7. The composed representation $\rho_{1} \hat{o} \rho_{2}$ is well-defined, i.e., one has that

(i) the definition of $\left(\rho_{1} \hat{o} \rho_{2}\right)_{I}$ is independent of the particular choices made, $\forall I \subset S^{1}$; and

(ii) if $I \subseteq J$ then $\left.\left(\rho_{1} \hat{o} \rho_{2}\right)_{J}\right|_{\text {, } /(I)}=\left(\rho_{1} \hat{o} \rho_{2}\right)_{I}$.

Proof. Ad (i). First, let $\rho_{1}$ and $\rho_{2}$ be localized in $I_{0}$ and let $I$ be such that $\left(I_{0} \cup I\right)^{-}$ does not cover $S^{1}$ : If $I \cap I_{0}=\emptyset$, then for any interval $J \supseteq I \cup I_{0}$ we have

$$
\left.\rho_{i, J}\right|_{\mathscr{\ell}(I)}=\left.\rho_{i, I}\right|_{. \mathscr{C}(I)}=\left.\mathrm{id}\right|_{. \ell(I)}, \quad i=1,2
$$

so that $\rho_{1, J} \circ \rho_{2, J}(A)=A, \forall A \in \mathscr{A}(I)$. So let us assume that $I \cup I_{0}$ is an interval. If $J, \tilde{J}$ are intervals containing $I \cup I_{0}$ then the interval $J \cap \tilde{J}$ also contains $I \cup I_{0}$ so that

$$
\begin{aligned}
\left.\rho_{1, J} \circ \rho_{2, J}\right|_{. \mathscr{C}(I)} & =\rho_{1, J \cap \tilde{J}} \circ \rho_{2,\left.J \cap \tilde{J}\right|_{. \mathscr{C}(I)}} \\
& =\left.\rho_{1, \tilde{J}} \circ \rho_{2, \tilde{J}}\right|_{\mathscr{L}(I)}
\end{aligned}
$$

and the definition of $\left(\rho_{1} \hat{\circ} \rho_{2}\right)_{I}$ is independent of the particular choice of $J \supseteq I \cup I_{0}$.

Next, let $I$ be such that $\left(I_{0} \cup I\right)^{-}$covers $S^{1}$. In the definition of $\left(\rho_{1} \hat{\circ} \rho_{2}\right)$ we chose $\tilde{I}_{0} \subseteq I_{0}$ small enough such that $\left(\tilde{I}_{0} \cup I\right)^{-}$does not cover $S^{1}$ and $J \supseteq \tilde{I}_{0} \cup I$. Clearly, by the first part of the proof, $\left.\tilde{\rho}_{1, J} \circ \tilde{\rho}_{2, J}\right|_{\mathscr{R}_{(I)}}$ is independent of the particular choice of $J \supseteq \tilde{I}_{0} \cup I$. It remains to check that choosing another interval $\hat{I}_{0} \subseteq I_{0}$ such that $\left(\hat{I}_{0} \cup I\right)^{-}$does not cover $S^{1}$ and representations $\hat{\rho}_{1}, \hat{\rho}_{2}$ localized in $\hat{I}_{0}$ together with intertwiners $\Gamma_{\rho_{1} \hat{\rho}_{1}}, \Gamma_{\rho_{2} \hat{\rho}_{2}}$ will leave $\left(\rho_{1} \hat{o} \rho_{2}\right)_{I}$ unchanged.

We start by showing that choosing $\hat{I}_{0} \subseteq \tilde{I}_{0}$ and $\hat{\rho}_{1}, \hat{\rho}_{2}$ localized in $\hat{I}_{0}$ does not change $\left(\rho_{1} \hat{\circ} \rho_{2}\right)_{I}$. The two possible definitions of $\left(\rho_{1} \hat{o} \rho_{2}\right)_{I}$ are

a) $\quad\left(\rho_{1} \hat{\circ} \rho_{2}\right)_{I}(\cdot)=\Gamma_{\rho_{1} \tilde{\rho}_{1}} \tilde{\rho}_{1, I_{0}}\left(\Gamma_{\rho_{2} \tilde{\rho}_{2}}\right) \tilde{\rho}_{1, J} \circ \tilde{\rho}_{2, J}(\cdot) \tilde{\rho}_{1, I_{0}}\left(\Gamma_{\rho_{2} \tilde{\rho}_{2}}\right)^{*} \Gamma_{\rho_{1}, \tilde{\rho}_{1}}^{*}$,

$$
=\rho_{1, I_{0}}\left(\Gamma_{\rho_{2} \tilde{\rho}_{2}}\right) \Gamma_{\rho_{1} \tilde{\rho}_{1}} \tilde{\rho}_{1, J} \circ \tilde{\rho}_{2, J}(\cdot) \Gamma_{\rho_{1} \tilde{\rho}_{1}}^{*} \rho_{1, I_{0}}\left(\Gamma_{\rho_{2} \tilde{\rho}_{2}}\right)^{*} \text {. }
$$

b) $\quad\left(\rho_{1} \hat{\circ} \rho_{2}\right)_{I}(\cdot)=\Gamma_{\rho_{1} \hat{\rho}_{1}} \hat{\rho}_{1, I_{0}}\left(\Gamma_{\rho_{2} \hat{\rho}_{2}}\right) \hat{\rho}_{1, J} \circ \hat{\rho}_{2, J}(\cdot) \hat{\rho}_{1, I_{0}}\left(\Gamma_{\rho_{2} \hat{\rho}_{2}}\right)^{*} \Gamma_{\rho_{1} \hat{\rho}_{1}}^{*}$,

$$
=\rho_{1, I_{0}}\left(\Gamma_{\rho_{2} \hat{\rho}_{2}}\right) \Gamma_{\rho_{1} \hat{\rho}_{1}} \hat{\rho}_{1, J} \circ \hat{\rho}_{2, J}(\cdot) \Gamma_{\rho_{1} \hat{\rho}_{1}}^{*} \rho_{1, I_{0}}\left(\Gamma_{\rho_{2} \hat{\rho}_{2}}\right)^{*} \text {. }
$$

They coincide if and only if

$$
\tilde{\rho}_{1, J} \circ \tilde{\rho}_{2, J}(\cdot)=\operatorname{ad}\left(\Gamma_{\rho_{1} \tilde{\rho}_{1}} \rho_{1, I_{0}}\left(\Gamma_{\rho_{2} \tilde{\rho}_{2}}^{*}\right) \rho_{1, I_{0}}\left(\Gamma_{\rho_{2} \hat{\rho}_{2}}\right) \Gamma_{\rho_{1} \hat{\rho}_{1}}\right) \hat{\rho}_{1, J} \circ \hat{\rho}_{2, J}(\cdot) .
$$

But,

$$
\rho_{1, I_{0}}\left(\Gamma_{\rho_{2} \tilde{\rho}_{2}}^{*}\right) \rho_{1, I_{0}}\left(\Gamma_{\rho_{2} \hat{\rho}_{2}}\right)=\rho_{1, I_{0}}\left(\Gamma_{\rho_{2} \tilde{\rho}_{2}}^{*} \Gamma_{\rho_{2} \hat{\rho}_{2}}\right)
$$

and $\Gamma_{\rho_{2} \tilde{\rho}_{2}}^{*} \Gamma_{\rho_{2} \hat{\rho}_{2}}=: \Gamma_{\tilde{\rho}_{2} \hat{\rho}_{2}}$ intertwines $\tilde{\rho}_{2}$ and $\hat{\rho}_{2}$; it is therefore localized in $\tilde{I}_{0}$. Furthermore,

$$
\Gamma_{\rho_{1} \tilde{\rho}_{1}}^{*} \rho_{1, I_{0}}\left(\Gamma_{\tilde{\rho}_{2} \hat{\rho}_{2}}\right) \Gamma_{\rho_{1} \hat{\rho}_{1}}=\tilde{\rho}_{1, I_{0}}\left(\Gamma_{\tilde{\rho}_{2} \hat{\rho}_{2}}\right) \Gamma_{\rho_{1} \tilde{\rho}_{1}}^{*} \Gamma_{\rho_{1} \hat{\rho}_{1}}
$$


and $\Gamma_{\rho_{1} \tilde{\rho}_{1}}^{*} \Gamma_{\rho_{1} \hat{\rho}_{1}}=: \Gamma_{\tilde{\rho}_{1} \hat{\rho}_{1}} \in \mathscr{A}\left(\tilde{I}_{0}\right)$. Since $\tilde{I}_{0} \subseteq I_{0}$ and $\tilde{I}_{0} \subseteq J$ it follows from the definition of $\tilde{\rho}_{1}$ that

$$
\tilde{\rho}_{1, I_{0}}\left(\Gamma_{\tilde{\rho}_{2} \hat{\rho}_{2}}\right)=\tilde{\rho}_{1, J}\left(\Gamma_{\tilde{\rho}_{2} \hat{\rho}_{2}}\right)
$$

so that the right-hand side of Eq. (4.2) is given by

$$
\begin{aligned}
\operatorname{ad}\left(\tilde{\rho}_{1, J}\left(\Gamma_{\tilde{\rho}_{2} \hat{\rho}_{2}}\right) \Gamma_{\tilde{\rho}_{1} \hat{\rho}_{1}}\right) \hat{\rho}_{1, J} \circ \hat{\rho}_{2, J}(\cdot) & =\operatorname{ad} \tilde{\rho}_{1, J}\left(\Gamma_{\tilde{\rho}_{2} \hat{\rho}_{2}}\right)\left(\tilde{\rho}_{1, J} \circ \hat{\rho}_{2, J}\right)(\cdot) \\
& =\tilde{\rho}_{1, J}\left(\operatorname{ad}\left(\Gamma_{\tilde{\rho}_{2} \hat{\rho}_{2}}\right) \hat{\rho}_{2, J}(\cdot)\right) \\
& =\tilde{\rho}_{1, J}\left(\tilde{\rho}_{2, J}(\cdot)\right)=\tilde{\rho}_{1, J} \circ \tilde{\rho}_{2, J}(\cdot)
\end{aligned}
$$

and this proves our assertion.

We can now choose the localization region $\tilde{I}_{0}$ of $\tilde{\rho}_{1}, \tilde{\rho}_{2}$ so small that it does not intersect $I$. It then follows that

$$
\left.\tilde{\rho}_{1, J} \circ \tilde{\rho}_{2, J}\right|_{\mathscr{C}(I)}=\tilde{\rho}_{1, I} \circ \tilde{\rho}_{2, I}=\left.\mathrm{id}\right|_{\mathscr{C}(I)}
$$

and, consequently,

$$
\left(\rho_{1} \hat{\circ} \rho_{2}\right)_{I}(\cdot)=\left.\operatorname{ad}\left(\Gamma_{\rho_{1} \tilde{\rho}_{1}} \tilde{\rho}_{1, I_{0}}\left(\Gamma_{\rho_{2} \tilde{\rho}_{2}}\right)\right)(\cdot)\right|_{\mathscr{C}(I)} .
$$

It remains to show that the map $\operatorname{ad}\left(\Gamma_{\rho_{1} \tilde{\rho}_{1}} \tilde{\rho}_{1, I_{0}}\left(\Gamma_{\rho_{2} \tilde{\rho}_{2}}\right)\right)$ is independent of the choice of $\tilde{I}_{0}, \tilde{\rho}_{1}$ and $\tilde{\rho}_{2}$ which fulfill the condition $\tilde{I}_{0} \cap I=\emptyset$. Choose $\hat{I}_{0}$ such that $\hat{I}_{0} \cap I=\emptyset$ and $\hat{\rho}_{1}, \hat{\rho}_{2}$ localized in $\hat{I}_{0}$. There exists then an interval $J_{0} \subset S^{1}$ such that $\tilde{I}_{0} \cup \hat{I}_{0} \subseteq J_{0}$, $J_{0} \cap I=\emptyset$. The map

$$
\left.\operatorname{ad}\left(\Gamma_{\rho_{1} \tilde{\rho}_{1}} \tilde{\rho}_{1, I_{0}}\left(\Gamma_{\rho_{2} \tilde{\rho}_{2}}\right)\right)\right|_{\mathscr{A}(I)}=\left.\operatorname{ad}\left(\rho_{1, I_{0}}\left(\Gamma_{\rho_{2} \tilde{\rho}_{2}}\right) \Gamma_{\rho_{1} \tilde{\rho}_{1}}\right)\right|_{\mathscr{C}(I)}
$$

is independent of the particular choice of $\tilde{I}_{0}$ if and only if

$$
\left.\operatorname{ad}\left(\Gamma_{\rho_{1} \hat{\rho}_{1}}^{*} \rho_{1, I_{0}}\left(\Gamma_{\rho_{2} \hat{\rho}_{2}}^{*}\right) \rho_{1, I_{0}}\left(\Gamma_{\rho_{2}, \hat{\rho}_{2}}\right) \Gamma_{\rho_{1} \tilde{\rho}_{1}}\right)\right|_{\mathscr{B}(I)}=\left.\mathrm{id}\right|_{\mathscr{L}(I)} .
$$

But

$$
\begin{aligned}
\Gamma_{\rho_{1} \hat{\rho}_{1}}^{*} \rho_{1, I_{0}}\left(\Gamma_{\rho_{2} \hat{\rho}_{2}}^{*}\right) \rho_{1, I_{0}}\left(\Gamma_{\rho_{2} \tilde{\rho}_{2}}\right) \Gamma_{\rho_{1} \tilde{\rho}_{1}} & =\Gamma_{\rho_{1} \hat{\rho}_{1}}^{*} \rho_{1, I_{0}}\left(\Gamma_{\rho_{2} \hat{\rho}_{2}}^{*} \Gamma_{\rho_{2} \tilde{\rho}_{2}}\right) \Gamma_{\rho_{1} \tilde{\rho}_{1}} \\
& =\Gamma_{\rho_{1} \hat{\rho}_{1}}^{*} \rho_{1, I_{0}}\left(\Gamma_{\tilde{\rho}_{2} \hat{\rho}_{2}}\right) \Gamma_{\rho_{1} \tilde{\rho}_{1}}
\end{aligned}
$$

where $\Gamma_{\hat{\rho}_{2} \tilde{\rho}_{2}}:=\Gamma_{\rho_{2} \hat{\rho}_{2}}^{*} \Gamma_{\rho_{2} \tilde{\rho}_{2}}$ intertwines $\hat{\rho}_{2}$ and $\tilde{\rho}_{2}$ and hence belongs to $\mathscr{A}\left(J_{0}\right)$. Furthermore,

$$
\begin{aligned}
\Gamma_{\rho_{1} \hat{\rho}_{1}}^{*} \rho_{1, I_{0}}\left(\Gamma_{\hat{\rho}_{2} \tilde{\rho}_{2}}\right) \Gamma_{\rho_{1} \tilde{\rho}_{1}} & =\hat{\rho}_{1, I_{0}}\left(\Gamma_{\hat{\rho}_{2} \tilde{\rho}_{2}}\right) \Gamma_{\rho_{1} \hat{\rho}_{1}}^{*} \Gamma_{\rho_{1} \tilde{\rho}_{1}} \\
& =\hat{\rho}_{1, J_{0}}\left(\Gamma_{\hat{\rho}_{2} \tilde{\rho}_{2}}\right) \Gamma_{\hat{\rho}_{1} \tilde{\rho}_{1}}
\end{aligned}
$$

where $\Gamma_{\hat{\rho}_{1} \tilde{\rho}_{1}}:=\Gamma_{\rho_{1} \hat{\rho}_{1}}^{*} \Gamma_{\rho_{1} \tilde{\rho}_{1}} \in \mathscr{A}\left(J_{0}\right)$, just as before. Hence,

$$
\hat{\rho}_{1, I_{0}}\left(\Gamma_{\hat{\rho}_{2} \tilde{\rho}_{2}}\right) \Gamma_{\hat{\rho}_{1} \tilde{\rho}_{1}}=\hat{\rho}_{1, J_{0}}\left(\Gamma_{\hat{\rho}_{2} \tilde{\rho}_{2}}\right) \Gamma_{\hat{\rho}_{1} \tilde{\rho}_{1}} \in \mathscr{A}\left(J_{0}\right)
$$

and since by locality $\mathscr{A}\left(J_{0}\right) \subseteq \mathscr{A}(I)^{\prime}$ this concludes the proof of the assertion and of (i).

Proof of (ii). There are three cases to be considered.

(a) If $I \subseteq J$ and $J \cup I_{0} \subseteq \tilde{J}$ then

$$
\begin{aligned}
& \left(\rho_{1} \hat{\circ} \rho_{2}\right)_{I}=\rho_{1, \tilde{J}} \circ \rho_{2, \tilde{J}}, \\
& \left(\rho_{1} \hat{\circ} \rho_{2}\right)_{J}=\rho_{1, \tilde{J}} \circ \rho_{2, \tilde{J}}
\end{aligned}
$$

and the assertion is obvious. 
(b) If $I \subseteq J$ and $\left(I \cup I_{0}\right)^{-}$covers $S^{1}$ so that $\left(J \cup I_{0}\right)^{-}$also covers $S^{1}$, then choosing $\tilde{I}_{0} \subseteq I_{0}$ such that there exists $\tilde{J} \supset J \cup \tilde{I}_{0}$ and $\tilde{\rho}_{1}$, $\tilde{\rho}_{2}$ localized in $\tilde{I}_{0}$ we have

$$
\begin{aligned}
& \left(\rho_{1} \hat{\circ} \rho_{2}\right)_{J}(\cdot)=\Gamma_{\rho_{1} \tilde{\rho}_{1}} \tilde{\rho}_{1, I_{0}}\left(\Gamma_{\rho_{2} \tilde{\rho}_{2}}\right) \tilde{\rho}_{1, \tilde{J}} \circ \tilde{\rho}_{2, \tilde{J}}(\cdot) \tilde{\rho}_{1, I_{0}}\left(\Gamma_{\rho_{2} \tilde{\rho}_{2}}^{*}\right) \Gamma_{\rho_{1} \tilde{\rho}_{1}}^{*}, \\
& \left(\rho_{1} \hat{\circ} \rho_{2}\right)_{I}(\cdot)=\Gamma_{\rho_{1} \tilde{\rho}_{1}} \tilde{\rho}_{1, I_{0}}\left(\Gamma_{\rho_{2} \tilde{\rho}_{2}}\right) \tilde{\rho}_{1, \tilde{J}} \circ \tilde{\rho}_{2, \tilde{J}}(\cdot) \tilde{\rho}_{1, I_{0}}\left(\Gamma_{\rho_{2} \tilde{\rho}_{2}}^{*}\right) \Gamma_{\rho_{1} \tilde{\rho}_{1}}^{*},
\end{aligned}
$$

the assertion is again immediate.

(c) If $I \subseteq J, I \cup I_{0} \subseteq \tilde{J}_{1}$ and $\left(J \cup I_{0}\right)^{-}$covers $S^{1}$ then choose $\tilde{I}_{0} \subset I_{0}$ such that there exists an interval $\tilde{J}_{2} \supseteq \tilde{I}_{0} \cup J$ and $\tilde{I}_{0} \cap J=\emptyset$. By definition of $\left(\rho_{1} \hat{\circ} \rho_{2}\right)_{J}$,

$$
\begin{aligned}
\left(\rho_{1} \hat{\circ} \rho_{2}\right)_{J} & =\left.\operatorname{ad}\left(\Gamma_{\rho_{1} \tilde{\rho}_{1}} \tilde{\rho}_{1, I_{0}}\left(\Gamma_{\rho_{2} \tilde{\rho}_{2}}\right)\right)\right|_{.,(J)} \\
& =\left.\operatorname{ad}\left(\rho_{1, I_{0}}\left(\Gamma_{\rho_{2} \tilde{\rho}_{2}}\right) \Gamma_{\rho_{1} \tilde{\rho}_{1}}\right)\right|_{.,(J)}
\end{aligned}
$$

for $\tilde{\rho}_{1}, \tilde{\rho}_{2}$ localized in $\tilde{I}_{0}$ and intertwiners $\Gamma_{\rho_{i} \tilde{\rho}_{i}}$ between $\rho_{i}$ and $\tilde{\rho}_{i}, i=1,2$. But

$$
\begin{aligned}
& \left(\rho_{1} \hat{\circ} \rho_{2}\right)_{I}=\left.\rho_{1, \tilde{J}_{1}} \circ \rho_{2, \tilde{J}_{1}}\right|_{\mathscr{A C}(I)} \\
& =\left.\rho_{1, \tilde{J}_{1}}\left(\Gamma_{\rho_{2} \tilde{\rho}_{2}} \tilde{\rho}_{2, \tilde{J}_{1}}(\cdot) \Gamma_{\rho_{2} \tilde{\rho}_{2}}^{*}\right)\right|_{\mathcal{L}(I)} \\
& =\left.\rho_{1, \tilde{J}_{1}}\left(\Gamma_{\rho_{2} \tilde{\rho}_{2}}\right) \Gamma_{\rho_{1} \tilde{\rho}_{1}} \tilde{\rho}_{1, \tilde{J}_{1}} \circ \tilde{\rho}_{2, \tilde{J}_{1}}(\cdot) \Gamma_{\rho_{1} \tilde{\rho}_{1}}^{*} \rho_{1, \tilde{J}_{1}}\left(\Gamma_{\rho_{2} \tilde{\rho}_{2}}^{*}\right)\right|_{. \not(I)} \\
& =\left.\operatorname{ad}\left(\rho_{1, I_{0}}\left(\Gamma_{\rho_{2} \tilde{\rho}_{2}}\right) \Gamma_{\rho_{1} \tilde{\rho}_{1}}\right) \circ \tilde{\rho}_{1, \tilde{J}_{1}} \circ \tilde{\rho}_{2, \tilde{J}_{1}}(\cdot)\right|_{, \not L(I)} \\
& =\left.\operatorname{ad}\left(\rho_{1, I_{0}}\left(\Gamma_{\rho_{2} \tilde{\rho}_{2}}\right) \Gamma_{\rho_{1} \tilde{\rho}_{1}}\right)\right|_{, /(I)},
\end{aligned}
$$

and this completes the proof of (ii) and of the lemma.

Lemma 4.8. Let $\rho_{1}$ and $\rho_{2}$ be two representations of . $b_{0}$ localized in $I_{0}$.

(i) The representation $\rho_{1} \hat{o} \rho_{2}$ of $A_{0}$ is localized in any interval $I_{1} \ni I_{0}$.

(ii) Let $\tilde{\rho}_{1}, \tilde{\rho}_{2}$ be representations of $\mathscr{C}_{0}$ unitarily equivalent to $\rho_{1}, \rho_{2}$ respectively and localized in a common interval $I_{1}$. Then

$$
\rho_{1} \hat{\circ} \rho_{2} \cong \tilde{\rho}_{1} \hat{\circ} \tilde{\rho}_{2} \text {. }
$$

(iii) If, moreover, there exists an interval $J \supseteq I_{0} \cup I_{1}$ and $\Gamma_{\rho_{1} \tilde{\rho}_{1}}, \Gamma_{\rho_{2} \tilde{\rho}_{2}}$ are intertwiners between $\rho_{1}$ and $\tilde{\rho}_{1}$, resp., $\rho_{2}$ and $\tilde{\rho}_{2}$, then

$$
\Gamma_{\rho_{1} \hat{o} \rho_{2}, \tilde{\rho}_{1} \hat{o} \tilde{\rho}_{2}}:=\Gamma_{\rho_{1} \tilde{\rho}_{1}} \tilde{\rho}_{1, J}\left(\Gamma_{\rho_{2} \tilde{\rho}_{2}}\right) \in \mathscr{A}(J)
$$

intertwines $\rho_{1} \hat{\circ} \rho_{2}$ and $\tilde{\rho}_{1} \hat{o} \tilde{\rho}_{2}$.

(iv) If $\rho_{1}$ is localized in $I_{1} \subseteq I_{0}, \rho_{2}$ in $I_{2} \subseteq I_{0}$ and $I_{1} \cap I_{2}=\emptyset$ then

$$
\rho_{1} \hat{\circ} \rho_{2}=\rho_{2} \hat{o} \rho_{1} \text {. }
$$

(v) The representation $\rho_{1} \hat{o} \rho_{2}$ is Moebius covariant.

Proof. Ad (i). Let $I_{1}$ be any interval strictly containing $I_{0}, I_{1} \supset \supset I_{0}$. It then follows that $\left(I_{1}^{\prime} \cup I_{0}\right)^{-}$does not cover $S^{1}$ so that

$$
\left(\rho_{1} \hat{\circ} \rho_{2}\right)_{I_{1}^{\prime}}(\cdot)=\left.\rho_{1, J} \circ \rho_{2, J}(\cdot)\right|_{, \ell\left(I_{1}^{\prime}\right)}
$$

for some $J \supseteq I_{1}^{\prime} \cup I_{0}$. However, since $\rho_{1}$ and $\rho_{2}$ are localized in $I_{0}$, it follows that

$$
\left.\rho_{1, J} \circ \rho_{2, J}(\cdot)\right|_{, \ell\left(I_{1}^{\prime}\right)}=\left.\mathrm{id}\right|_{\ell\left(I_{1}^{\prime}\right)} \text {. }
$$

Ad (iii). It is sufficient to consider the case of $I_{1} \subseteq I_{0}$ and $J=I_{0}$. We show that $\Gamma_{\rho_{1} \hat{\circ} \rho_{2}, \tilde{\rho}_{1} \hat{o} \tilde{\rho}_{2}} \in \mathscr{C}\left(I_{0}\right)$, as defined in (iii), intertwines $\left(\rho_{1} \hat{\circ} \rho_{2}\right)_{I}$ and $\left(\tilde{\rho}_{1} \hat{\circ} \tilde{\rho}_{2}\right)_{I}$, for each interval $I \subset S^{1}$. There are three possible situations. 
(a) If $I \cup I_{0} \subseteq \tilde{J}$ for some interval $\tilde{J}$, then $I \cup I_{0} \cup I_{1} \subseteq \tilde{J}$, and hence

$$
\begin{aligned}
\left(\rho_{1} \hat{\circ} \rho_{2}\right)_{I}(\cdot) \Gamma_{\rho_{1} \hat{\circ} \rho_{2}, \tilde{\rho}_{1} \hat{\circ} \tilde{\rho}_{2}} & =\rho_{1, \tilde{J}} \circ \rho_{2, \tilde{J}}(\cdot) \Gamma_{\rho_{1} \tilde{\rho}_{1}} \tilde{\rho}_{1, I_{0}}\left(\Gamma_{\rho_{2} \tilde{\rho}_{2}}\right) \\
& =\Gamma_{\rho_{1} \tilde{\rho}_{1}} \tilde{\rho}_{1, \tilde{J}} \circ \rho_{2, \tilde{J}}(\cdot) \tilde{\rho}_{1, \tilde{J}}\left(\Gamma_{\rho_{2} \tilde{\rho}_{2}}\right) \\
& =\Gamma_{\rho_{1} \tilde{\rho}_{1}} \tilde{\rho}_{1, \tilde{J}}\left(\Gamma_{\rho_{2} \tilde{\rho}_{2}}\right) \tilde{\rho}_{1, \tilde{J}} \circ \tilde{\rho}_{2, \tilde{J}}(\cdot) \\
& =\Gamma_{\rho_{1} \hat{\circ} \rho_{2}, \tilde{\rho}_{1} \hat{\circ} \tilde{\rho}_{2}}\left(\tilde{\rho}_{1} \hat{\circ} \tilde{\rho}_{2}\right)_{I}(\cdot) .
\end{aligned}
$$

(b) If $\left(I \cup I_{0}\right)^{-}$covers $S^{1}$ but $I \cup I_{1} \subseteq \tilde{J}$ for some interval $\tilde{J}$, then by definition of $\left(\rho_{1} \hat{o} \rho_{2}\right)_{I}$ and of $\left(\tilde{\rho}_{1} \hat{o} \tilde{\rho}_{2}\right)_{I}$,

$$
\begin{aligned}
\left(\rho_{1} \hat{\circ} \rho_{2}\right)_{I} & =\operatorname{ad}\left(\Gamma_{\rho_{1} \tilde{\rho}_{1}} \tilde{\rho}_{1, I_{0}}\left(\Gamma_{\rho_{2} \tilde{\rho}_{2}}\right)\right) \tilde{\rho}_{1, \tilde{J}} \circ \tilde{\rho}_{2, \tilde{J}}(\cdot) \\
\left(\tilde{\rho}_{1} \hat{\circ} \tilde{\rho}_{2}\right) & =\tilde{\rho}_{1, \tilde{J}} \circ \tilde{\rho}_{2, \tilde{J}}(\cdot)
\end{aligned}
$$

and the claim is immediate.

(c) If $\left(I \cup I_{0}\right)^{-}$and $\left(I \cup I_{1}\right)^{-}$cover $S^{1}$, pick $\hat{I}_{1} \subseteq I_{1}$ such that $I \cup \hat{I}_{1} \subseteq \tilde{J}$ for some interval $\tilde{J}$. Let $\hat{\rho}_{1}, \hat{\rho}_{2}$ be unitarily equivalent to $\tilde{\rho}_{1}, \tilde{\rho}_{2}$ and $\Gamma_{\tilde{\rho}_{1} \hat{\rho}_{1}}, \Gamma_{\tilde{\rho}_{2} \hat{\rho}_{2}}$ be the corresponding intertwiners. It then follows that $\Gamma_{\rho_{i} \tilde{\rho}_{\imath}} \cdot \Gamma_{\tilde{\rho}_{i} \hat{\rho}_{2}}$ intertwines $\rho_{i}$ and $\hat{\rho}_{i}$, $i=1,2$.

By definition,

$$
\begin{aligned}
& \left(\rho_{1} \hat{\circ} \rho_{2}\right)_{I}=\left.\operatorname{ad}\left(\Gamma_{\rho_{1} \tilde{\rho}_{1}} \Gamma_{\tilde{\rho}_{1} \hat{\rho}_{1}} \hat{\rho}_{1, I_{0}}\left(\Gamma_{\rho_{2} \tilde{\rho}_{2}} \Gamma_{\tilde{\rho}_{2} \hat{\rho}_{2}}\right)\right) \circ \hat{\rho}_{1, \tilde{J}} \circ \hat{\rho}_{2, \tilde{J}}\right|_{\mathscr{\ell}(I)} \\
& =\left.\operatorname{ad}\left(\Gamma_{\rho_{1} \tilde{\rho}_{1}} \tilde{\rho}_{1, I_{0}}\left(\Gamma_{\rho_{2} \tilde{\rho}_{2}} \Gamma_{\tilde{\rho}_{2} \hat{\rho}_{2}}\right) \Gamma_{\rho_{1} \hat{\rho}_{1}}\right) \circ \hat{\rho}_{1, \tilde{J}} \circ \hat{\rho}_{2, \tilde{J}}\right|_{. \ell(I)} \\
& =\left.\operatorname{ad}\left(\Gamma_{\rho_{1} \tilde{\rho}_{1}} \tilde{\rho}_{1, I_{0}}\left(\Gamma_{\rho_{2} \tilde{\rho}_{2}}\right) \tilde{\rho}_{1, I_{0}}\left(\Gamma_{\tilde{\rho}_{2} \hat{\rho}_{2}}\right) \Gamma_{\tilde{\rho}_{1} \hat{\rho}_{1}}\right) \circ \hat{\rho}_{1, \tilde{J}} \circ \hat{\rho}_{2, \tilde{J}}\right|_{\mathscr{l}(I)} \\
& =\left.\operatorname{ad}\left(\Gamma_{\rho_{1} \tilde{\rho}_{1}} \tilde{\rho}_{1, I_{0}}\left(\Gamma_{\rho_{2} \tilde{\rho}_{2}}\right)\right) \circ \operatorname{ad}\left(\tilde{\rho}_{1, I_{0}}\left(\Gamma_{\tilde{\rho}_{2} \hat{\rho}_{2}}\right) \Gamma_{\tilde{\rho}_{1} \hat{\rho}_{1}}\right) \circ \hat{\rho}_{1, \tilde{J}} \circ \tilde{\rho}_{2, \tilde{J}}\right|_{\mathscr{H}(I)} \\
& =\left.\operatorname{ad}\left(\Gamma_{\rho_{1} \hat{\circ} \rho_{2}, \tilde{\rho}_{1} \hat{\circ} \tilde{\rho}_{2}}\right) \circ \operatorname{ad}\left(\Gamma_{\tilde{\rho}_{1} \hat{\rho}_{1}} \hat{\rho}_{1, I_{1}}\left(\Gamma_{\tilde{\rho}_{2} \hat{\rho}_{2}}\right)\right) \circ \hat{\rho}_{1, \tilde{J}} \circ \hat{\rho}_{2, \tilde{J}}\right|_{\mathscr{L}(I)} \\
& =\operatorname{ad}\left(\Gamma_{\rho_{1} \hat{\circ} \rho_{2}, \tilde{\rho}_{1} \hat{\circ} \tilde{\rho}_{2}}\right)\left(\tilde{\rho}_{1} \hat{\circ} \tilde{\rho}_{2}\right)_{I} \text {. }
\end{aligned}
$$

This completes the proof of assertion (iii).

Ad (ii). If $I_{0} \cup I_{1}$ is contained in some interval, we are done by (iii). If not, choose $\hat{I}$ such that $I_{0} \cup \hat{I}$ and $\hat{I} \cup I_{1}$ are contained in some intervals, $\hat{\rho}_{1}, \hat{\rho}_{2}$ localized in $\hat{I}$ unitarily equivalent to $\rho_{1}, \rho_{2}$. We can then apply assertion (iii) twice to conclude the proof of (ii).

Ad (iv). We check that $\left(\rho_{1} \hat{o} \rho_{2}\right)_{I}=\left(\rho_{2} \hat{o} \rho_{1}\right)_{I}$ for each interval $I \subseteq S^{1}$. If $I \cup I_{0}$ is contained in some interval $J \subset S^{1}$, then the proof follows as in Lemma 2.2 of ref. [64]. If $\left(I \cup I_{0}\right)^{-}$covers $S^{1}$, choose $\hat{I}_{1}, \hat{I}_{2} \subseteq I_{0}$ and $\hat{\rho}_{1}, \hat{\rho}_{2}$ localized in $\hat{I}_{1}, \hat{I}_{2}$, unitarily equivalent to $\rho_{1}, \rho_{2}$ respectively, satisfying the following properties:

(a) $\hat{I}_{1} \cap \hat{I}_{2}=\emptyset$,

(b) there exists $\tilde{I}_{1} \supseteq \tilde{I}_{1} \cup \hat{I}_{1}, \tilde{I}_{2} \supseteq I_{2} \cup \hat{I}_{2}$ such that $\tilde{I}_{1} \cap \tilde{I}_{2}=\emptyset$.

(c) There exists $\hat{I}_{0} \supseteq \hat{I}_{1} \cup \hat{I}_{2}$ and $\tilde{J} \supseteq I \cup \hat{I}_{0}$.

If $\Gamma_{\rho_{1} \hat{\rho}_{1}}, \Gamma_{\rho_{2} \hat{\rho}_{2}}$ are intertwiners between $\rho_{1}, \hat{\rho}_{1}$ and $\rho_{2}, \hat{\rho}_{2}$, then

$$
\begin{aligned}
& \Gamma_{\rho_{1} \hat{\rho}_{1}} \in \mathcal{A}\left(\tilde{I}_{1}\right) \subseteq \mathscr{A}\left(\tilde{I}_{2}\right)^{\prime}, \quad \Gamma_{\rho_{2} \hat{\rho}_{2}} \in \mathcal{A}\left(\tilde{I}_{2}\right), \\
& \hat{\rho}_{1, I_{0}}\left(\Gamma_{\rho_{2} \hat{\rho}_{2}}\right)=\hat{\rho}_{1, \tilde{I}_{2}}\left(\Gamma_{\rho_{2} \hat{\rho}_{2}}\right)=\Gamma_{\rho_{2} \hat{\rho}_{2}}, \\
& \hat{\rho}_{2, I_{0}}\left(\Gamma_{\rho_{1} \hat{\rho}_{1}}\right)=\hat{\rho}_{2, \tilde{I}_{1}}\left(\Gamma_{\rho_{1} \hat{\rho}_{1}}\right)=\Gamma_{\rho_{1} \hat{\rho}_{1}},
\end{aligned}
$$


so that

$$
\begin{aligned}
\left(\rho_{1} \hat{\circ} \rho_{2}\right)_{I} & =\left.\operatorname{ad}\left(\Gamma_{\rho_{1} \hat{\rho}_{1}} \hat{\rho}_{1, I_{0}}\left(\Gamma_{\rho_{2} \hat{\rho}_{2}}\right)\right) \hat{\rho}_{1, \tilde{J}} \circ \hat{\rho}_{2, \tilde{J}}\right|_{1 / \ell(I)} \\
& =\operatorname{ad}\left(\Gamma_{\rho_{1} \hat{\rho}_{1}} \Gamma_{\rho_{2} \hat{\rho}_{2}}\right)\left(\hat{\rho}_{1} \circ \hat{\rho}_{2}\right)_{I} \\
& =\operatorname{ad}\left(\Gamma_{\rho_{2} \hat{\rho}_{2}} \Gamma_{\rho_{1} \hat{\rho}_{1}}\right)\left(\hat{\rho}_{2} \circ \hat{\rho}_{1}\right)_{I} \\
& =\left.\operatorname{ad}\left(\Gamma_{\rho_{2} \hat{\rho}_{2}} \hat{\rho}_{2, I_{0}}\left(\Gamma_{\rho_{1} \hat{\rho}_{1}}\right)\right) \hat{\rho}_{2, \tilde{J}} \circ \hat{\rho}_{1, \tilde{J}}\right|_{/ \ell(I)} \\
& =\left(\rho_{2} \hat{\circ} \rho_{1}\right)_{I},
\end{aligned}
$$

and this completes the proof of assertion (iv).

Ad (v). Let $\rho_{1}, \rho_{2}$ be localized in $I_{0}$ and let $I_{1} \ni I_{0}$ so that $\rho_{1} \hat{\circ} \rho_{2}$ is localized in $I_{1}$. Denote by $\mathscr{W}$ a neighborhood of the identity in $\widetilde{P S U}(1,1)$ such that if $\tilde{g} \in \mathscr{W}$ then $I_{1} \cup \tilde{g} \cdot I_{1}$ is contained in some interval $\tilde{J}$. It follows from locality and duality that

$$
\begin{aligned}
\Gamma_{\rho_{1} \hat{\circ} \rho_{2}}(\tilde{g}): & =\rho_{1 g, \tilde{J}}\left(\Gamma_{\rho_{2}}(\tilde{g})\right) \Gamma_{\rho_{1}}(\tilde{g}) \\
& =\Gamma_{\rho_{1}}(\tilde{g}) \rho_{1, \tilde{J}}\left(\Gamma_{\rho_{2}}(\tilde{g})\right)
\end{aligned}
$$

is well defined for all $\tilde{g} \in \mathscr{W}$. If $\tilde{g}_{1}, \tilde{g}_{2}$ and $\tilde{g}_{1} \cdot \tilde{g}_{2} \in \mathscr{W}$ then it follows from the definition of $\Gamma_{\rho_{1} \hat{\circ} \rho_{2}}\left(\tilde{g}_{1}\right), \Gamma_{\rho_{1} \hat{\jmath} \rho_{2}}\left(\tilde{g}_{2}\right)$ and $\Gamma_{\rho_{1} \hat{\circ} \rho_{2}}\left(\tilde{g}_{1} \cdot \tilde{g}_{2}\right)$ that they satisfy the cocycle identity

$$
\Gamma_{\rho_{1} \hat{\jmath} \rho_{2}}\left(\tilde{g}_{1} \cdot \tilde{g}_{2}\right)=\alpha_{g_{1}}\left(\Gamma_{\rho_{1} \hat{\circ} \rho_{2}}\left(\tilde{g}_{2}\right)\right) \Gamma_{\rho_{1} \hat{\circ} \rho_{2}}\left(\tilde{g}_{1}\right)
$$

Hence $\Gamma_{\rho_{1} \hat{\circ} \rho_{2}}(\tilde{g})$ extends to a cocycle on $\widetilde{P S U}(1 ; 1)$ by the original argument given in ref. [23], Sect. 8.

Define the shifted representations $\left(\rho_{1} \hat{\circ} \rho_{2}\right)_{g}$ by $\left(\rho_{1} \hat{\circ} \rho_{2}\right)_{g}:=\alpha_{g} \circ\left(\rho_{1} \hat{\circ} \rho_{2}\right) \circ \alpha_{g-1}$, that is,

$$
\left(\rho_{1} \hat{\circ} \rho_{2}\right)_{g, I}(\cdot)=\alpha_{g} \circ\left(\rho_{1} \hat{\circ} \rho_{2}\right)_{g^{-1} \cdot I} \alpha_{g^{-1}}(\cdot), \forall I \subseteq S^{1}
$$

To prove Moebius covariance of $\left(\rho_{1} \hat{o} \rho_{2}\right)$ it is sufficient to prove that the cocycle $\Gamma_{\rho_{1} \hat{o} \rho_{2}}(\tilde{g})$ intertwines $\rho_{1} \hat{o} \rho_{2}$ and $\left(\rho_{1} \hat{o} \rho_{2}\right)_{g}$ for $\tilde{g} \in \widetilde{P S U}(1 ; 1)$,

$$
\left(\rho_{1} \hat{\circ} \rho_{2}\right)_{g}(A) \Gamma_{\rho_{1} \hat{\mathrm{o}} \rho_{2}}(\tilde{g})=\Gamma_{\rho_{1} \hat{\mathrm{o}} \rho_{2}}(\tilde{g})\left(\rho_{1} \hat{\circ} \rho_{2}\right)(A) \quad \forall A \in \mathscr{C}_{0}
$$

It is sufficient to prove the intertwining property (4.3) for arbitrary intervals $I$ only in the special case of $\tilde{g} \in \mathscr{W}$ and $g \cdot I_{1} \subseteq I_{1}$ or $I_{1} \subseteq g \cdot I_{1}$. Namely, if $\tilde{g}$ is arbitrary, we write $\tilde{g}$ as a product $\tilde{g}_{1} \cdot \tilde{g}_{2} \ldots \tilde{g}_{n}$ of elements in $\mathscr{W}$ such that if $I_{k}:=g_{1} \ldots g_{k-1} \cdot I_{1}$ then either $g_{k} \cdot I_{k} \subseteq I_{k+1}$ or $g_{k} \cdot I_{k} \supseteq I_{k+1}$. By the cocycle property, we have the decomposition

$\Gamma_{\rho_{1} \hat{\circ} \rho_{2}}(\tilde{g})=\alpha_{g_{1} \ldots g_{n-1}}\left(\Gamma_{\rho_{1} \hat{\circ} \rho_{2}}\left(\tilde{g}_{n}\right)\right) \alpha_{g_{1} \ldots g_{n-2}}\left(\Gamma_{\rho_{1} \hat{\circ} \rho_{2}}\left(\tilde{g}_{n-1}\right)\right) \ldots \alpha_{g_{1}}\left(\Gamma_{\rho_{1} \hat{\circ} \rho_{2}}\left(\tilde{g}_{2}\right)\right) \Gamma_{\rho_{1} \hat{o} \rho_{2}}\left(\tilde{g}_{1}\right)$

and we derive the intertwining property for general $\tilde{g}$, by applying, step by step, the special case. Since the special case was in fact proven in (iii), the proof is complete. 


\section{IV.3. Haag-Kastler Subnets on Light-Rays}

Let $\mathscr{A}_{0}=\{\mathscr{A}(I)\}_{I \subset S^{1}}$ be a conformal net. We now construct subnets of $\mathscr{A}_{0}$ associated to light rays in Minkowski space and investigate their properties. The results of this section will be used in Sect. IV.5 to adapt classical results of Doplicher, Haag and Roberts (see refs. [13, 14]) to the present setting.

Pick a point $\alpha \in S^{1}$ and project stereographically $S^{1} \backslash\{\alpha\}$ onto $\mathbb{R}, \alpha$ representing the point at infinity. Let $p_{\alpha}: S^{1} \backslash\{\alpha\} \rightarrow \mathbb{R}$ denote this stereographic projection. Bounded intervals $I$ on $\mathbb{R}$ are in one-to-one correspondence with intervals $J=p_{\alpha}^{-1}(I)$ on $S^{1}$ such that the closure of $J$ does not contain $\alpha$. If we define

$$
\mathscr{A}_{\alpha}(I):=\mathscr{A}\left(p_{\alpha}^{-1}(I)\right) \text {, }
$$

where $I \subseteq \mathbb{R}$ is a bounded interval and

$$
\mathscr{C}_{\alpha}:=\left\{\mathscr{C}_{\alpha}(I) \mid I \subset \mathbb{R} \text { is bounded }\right\} \subseteq \mathscr{A}_{0},
$$

then $\mathscr{A}_{\alpha}$ is a local, isotone net on $\mathbb{R}$ and the $C^{*}$-inductive limit, $\overline{\mathscr{b}}_{\alpha}$, of $\mathscr{C}_{\alpha}$ is an algebra of quasi-local observables in the sense of Haag and Kastler [19]. Furthermore, $\bar{b}_{\alpha}$ is covariant under the Poincaré subgroup of the Moebius group which leaves the point $\alpha$ fixed (see Appendix I for details). Notice also that the unitary operators implementing the rotations of the circle on $\mathscr{H}$ provide isomorphisms between the different $C^{*}$-algebras $\overline{\mathscr{b}}_{\alpha} \alpha \in S^{1}$.

Let $\alpha \in S^{1}$ be fixed. For convenience, we adopt the following notation: if $I \subset \mathbb{R}$ is an interval (or any other set) we write $I_{S^{1}}$ for the inverse image of $I$ by $p_{\alpha}$, i.e.,

$$
I_{S^{1}}:=p_{\alpha}^{-1}(I)
$$

conversely, if $I \subset S^{1}, I_{\mathbb{R}}$ will denote its image in $\mathbb{R}$ under $p_{\alpha}, I_{\mathbb{R}}:=p_{\alpha}(I)$.

On $\mathbb{R}$, the complement of a bounded interval $I:=(a, b)$ is defined by $I^{\prime \mathbb{R}}:=$ $I_{<} \cup I_{>}, I_{<}:=(-\infty, a), I_{>}:=(b, \infty)$, and the algebra, $\mathscr{A}_{\alpha}\left(I^{\prime \mathbb{R}}\right)$ for the unbounded region $I_{<} \cup I_{>}$is defined as the $C^{*}$-algebra generated by all $\mathscr{A}_{\alpha}(J)$, such that $J$ is a bounded interval contained in $I_{>}$or $I_{<}$. Note that the weak closure $\mathscr{C}_{\alpha}\left(I_{\gtrless}\right)^{-w}$ of $\mathscr{A}_{\alpha}\left(I_{\gtrless}\right)$ is given by

$$
\mathscr{C}_{\alpha}\left(I_{\gtrless}\right)^{-w}=\mathscr{A}\left(p_{\alpha}^{-1}\left(I_{\gtrless}\right)\right)=\mathscr{A}\left(I_{\gtrless S^{1}}\right) .
$$

Given an isotone, local net of von Neumann algebras on $\mathbb{R}, \mathscr{A}=\{\mathscr{b}(I) \mid I \subset \mathbb{R}$ is a bounded interval $\}$ we define its dual net by

$$
\begin{aligned}
\mathscr{C}^{d}(I) & :=\mathscr{C}\left(I^{\prime} \mathbb{R}\right)^{\prime}, \\
\mathscr{C}^{d} & :=\left\{\mathscr{C}^{d}(I) \mid I \subset \mathbb{R} \text { is a bounded interval }\right\} .
\end{aligned}
$$

Clearly, a net $\mathscr{C}$ is local if and only if $\mathscr{b} \subseteq \mathscr{b}^{d}$.

Definition 4.9. (i) A local isotone net $\mathscr{A}$ on $\mathbb{R}$ is said to satisfy duality if $\mathscr{C}^{d}=\mathscr{A}$ holds.

(ii) A local isotone net $\mathscr{A}$ on $\mathbb{R}$ is said to satisfy essential duality if

$$
\mathscr{C}^{d d}=\mathscr{C}^{d}
$$

holds.

The following lemma is due to J. Roberts. 
Lemma 4.10. A local net $\mathscr{A}=\{\mathscr{C}(I)\}_{I \subset \mathbb{R}}$ on $\mathbb{R}$ satisfies essential duality if and only if $\mathscr{A}^{d}$ is local.

Proof. Since by the remark preceding Definition 4.9,

$$
\mathscr{C}^{d} \text { is local } \Leftrightarrow \mathscr{C}^{d} \subseteq \mathscr{C}^{d d} \text {, }
$$

we have to show that the converse inclusion $\mathscr{C}^{d} \supseteq \mathscr{b}^{d d}$ follows automatically if $\mathscr{b}$ is local. Now, by definition,

$$
\mathscr{f}\left(I^{\prime} \mathbb{R}\right)\left\{\bigcup_{I_{0} \subseteq I^{\prime} \mathbb{R}} \mathscr{f}\left(I_{0}\right)\right\}^{-n} \subseteq\left\{\bigcup_{I_{0} \subseteq I^{\prime} \mathbb{R}} \mathscr{f b}\left(I_{0}^{\prime} \mathbb{R}\right)^{\prime}\right\}^{-n},
$$

where the symbol ${ }^{-n}$ indicates norm closure and the inclusion follows by locality of the net $\mathscr{A}$. Taking commutants on both sides, we obtain

$$
\bigcap_{I_{0} \subseteq I^{\prime} \mathbb{R}} \mathscr{A}\left(I_{0}^{\prime} \mathbb{R}\right)^{\prime \prime} \subseteq \mathscr{A}\left(I^{\prime} \mathbb{R}\right)^{\prime}=\mathscr{C}^{d}(I) .
$$

But,

$$
\begin{aligned}
\mathscr{A}^{d d}(I): & =\mathscr{A}^{d}\left(I^{\prime} \mathbb{R}\right)^{\prime}=\left\{\bigcup_{I_{0} \subseteq I^{\prime} \mathbb{R}} \mathscr{A}^{d}\left(I_{0}\right)\right\}^{\prime} \\
& =\left\{\bigcup_{I_{0} \subseteq I^{\prime} \mathbb{R}} \mathscr{A}\left(I_{0}^{\prime \mathbb{R}}\right)^{\prime}\right\}^{\prime}=\bigcap_{I_{0} \subseteq I^{\prime} \mathbb{R}} \mathscr{A}\left(I_{0}^{\prime} \mathbb{R}\right)^{\prime \prime}
\end{aligned}
$$

and this completes the proof.

Theorem 4.11. Let $\mathscr{A}_{0}=\{\mathscr{A}(I)\}_{I \subset S^{1}}$ be a conformal net. Then $\mathscr{A}_{\alpha}$ satisfies essential duality, for any $\alpha \in S^{1}$.

Proof. By Lemma 4.10 it is sufficient to show that $\mathscr{b}_{\alpha}^{d}$ is local. Since $\mathscr{C}_{\alpha}\left(I^{\prime} \mathbb{R}\right)$ is the $C^{*}$-algebra generated by all $\mathscr{A}_{\alpha}(J) \subseteq \mathscr{A}_{\alpha}\left(I_{<}\right)$or $\mathscr{A}_{\alpha}^{\alpha}(J) \subseteq \mathscr{A}_{\alpha}\left(I_{>}\right)$for $J$ bounded, it follows that

$$
\begin{aligned}
\mathscr{C}_{\alpha}^{d}(I) & =\mathscr{C}_{\alpha}\left(I^{\prime} \mathbb{R}\right)^{\prime} \\
& =\left(\mathscr{C}_{\alpha}\left(I_{>}\right) \cup \mathscr{C}_{\alpha}\left(I_{<}\right)\right)^{\prime} \\
& =\mathscr{A}_{(}\left(I_{>S^{1}}\right)^{\prime} \cap \mathscr{A}_{(}\left(I_{<S^{1}}\right)^{\prime} \\
& =\mathscr{A}\left(\left(I_{>S^{1}}\right)^{\prime}\right) \cap \mathscr{A}\left(\left(I_{<S^{1}}\right)^{\prime}\right)
\end{aligned}
$$

by conformal duality. But as the following picture shows,
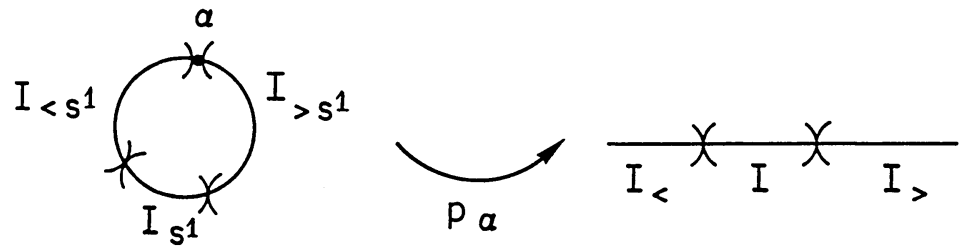

$$
\begin{aligned}
\mathscr{A}\left(\left(I_{>S^{1}}\right)^{\prime}\right) \cap \mathscr{C}\left(\left(I_{<S^{1}}\right)^{\prime}\right) & =\mathscr{A}\left(\left(I_{<} \cup I\right)_{S^{1}}\right) \cap \mathscr{A}\left(\left(I_{>} \cup I\right)_{S^{1}}\right) \\
& =\mathscr{A}_{\alpha}\left(I_{<} \cup I\right)^{-w} \cap \mathscr{A}_{\alpha}\left(I_{>} \cup I\right)^{-w},
\end{aligned}
$$


by Eq. (4.4). Hence

$$
\mathscr{C}_{\alpha}^{d}(I)=\mathscr{A}_{\alpha}\left(I_{<} \cup I\right)^{-w} \cap \mathscr{C}_{\alpha}\left(I_{>} \cup I\right)^{-w} .
$$

Now the proof of locality for the net $\mathscr{A}_{\alpha}^{d}$ is obvious. If $I_{1} \cap I_{2}=\emptyset$, we may assume that $I_{1}$ lies on the left of $I_{2}$ :

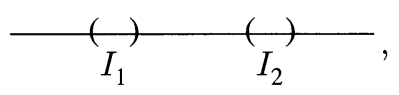

so that

$$
\begin{aligned}
& I_{1} \cup I_{1>} \supseteq I_{2} \cup I_{2>}, \\
& I_{1} \cup I_{1<} \subseteq I_{2} \cup I_{2<}
\end{aligned}
$$

But

$$
\begin{aligned}
\mathscr{C}_{\alpha}^{d}\left(I_{1}\right) & =\mathscr{A}\left(\left(I_{1} \cup I_{1<}\right)_{S^{1}}\right) \cap \mathscr{A}\left(\left(I_{1} \cup I_{1>}\right)_{S_{1}}\right) \\
& \subseteq \mathscr{A}\left(\left(I_{1} \cup I_{1<}\right)_{S^{1}}\right)
\end{aligned}
$$

and

$$
\begin{aligned}
\mathscr{C}_{\alpha}^{d}\left(I_{2}\right) & =\mathscr{A}\left(\left(I_{2} \cup I_{2<}\right)_{S^{1}}\right) \cap \mathscr{A}\left(\left(I_{2} \cup I_{2>}\right)_{S_{1}}\right) \\
& \subseteq \mathscr{A}\left(\left(I_{2} \cup I_{2>}\right)_{S^{1}}\right) \subseteq \mathscr{A}\left(\left(I_{1} \cup I_{1<}\right)_{S^{1}}\right)^{\prime}
\end{aligned}
$$

by locality of the conformal net $\mathscr{C}_{0}$. This shows that the net $\mathscr{b}_{\alpha}^{d}$ is local and completes the proof of the theorem.

Remarks 4.12. (i) Essential duality was introduced by Roberts [65] in his study of spontaneously broken internal gauge symmetries in algebraic quantum field theory.

(ii) If $\mathscr{b}_{\alpha}=\mathscr{b}_{\alpha}^{d}$ then the net $\mathscr{A}_{\alpha}$ is dual. This situation arises for a massless scalar field [53,31] or for the local algebras generated by a $U(1)$-current [30,31], for example. However, in general this equality does not hold; counter examples are the local algebras obtained from a Virasoro algebra of central charge $c>1$ [31].

(iii) It follows from Eqs. (4.4) and (4.5) that $\mathscr{A}_{\alpha}^{d} \subseteq \mathscr{A}_{0}$.

Theorem 4.13. Let $\rho$ be a covariant representation of $\mathscr{C}_{0}$ localized in an interval $I_{0} \subset S^{1}$ and $\alpha \in S^{1} \backslash I_{0}^{-}$.

(i) The restriction of $\rho$ to $\mathscr{C}_{\alpha}$ extends to an endomorphism $\rho_{\alpha}$ of $\overline{\mathscr{b}}_{\alpha}$ localized in $I_{0 \mathbb{R}}$.

(ii) The restriction of $\rho$ to $\mathscr{C}_{\alpha}^{d}$ extends to an endomorphism $\rho_{\alpha}^{d}$ of $\overline{\mathscr{b}}_{\alpha}^{d}$ localized in $I_{0 \mathbb{R}}$ such that $\left.\rho_{\alpha}^{d}\right|_{\bar{b}_{\alpha}}=\rho_{\alpha}$.

(iii) $\rho_{\alpha}$ and $\rho_{\alpha}^{d}$ are covariant under the Poincaré subgroup of the Moebius group leaving the point $\alpha$ fixed.

Proof. (i) Since for every bounded interval $I \subset \mathbb{R}$ and every

$$
A \in \mathscr{A}_{\alpha}(I)=\mathscr{A}\left(I_{S^{1}}\right), \quad \rho_{\alpha}(A):=\rho_{I_{S^{1}}}(A)
$$

is well defined and norm-continuous, $\rho_{\alpha}$ extends to $\bar{\phi}_{\alpha}$. Let $J$ be a bounded interval in $\mathbb{R}$ containing $I_{0 \mathbb{R}}$ and $I$. By locality and conformal duality,

$$
\rho_{\alpha}(A) \in \mathscr{b}\left(J_{S^{1}}\right)=\mathscr{A}_{\alpha}(J)
$$

since $\rho$ is localized in $I_{0}$. Hence $\rho_{\alpha}$ is an endomorphism of $\overline{\mathscr{b}}_{\alpha}$. 
(ii) If $I \subset \mathbb{R}$ is bounded and $A \in \mathscr{\ell}_{\alpha}^{d}(I)$ then $A \in \mathscr{A}\left(\left(I \cup I_{<}\right)_{S^{1}}\right) \cap \mathscr{A}\left(\left(I \cup I_{>}\right)_{S^{1}}\right)$. Hence

$$
\rho_{\alpha}^{d}(A):=\rho_{\left(I \cup I_{<)^{1}}\right.}(A)=\rho_{\left(I \cup I_{>}\right)_{S} 1}(A)
$$

is well-defined and extends by norm continuity to $\overline{\mathscr{b}}_{\alpha}^{d}$.

To prove the second equality, we can use the following arguments. Let $I$ and $J$ be intervals on $S^{1}$ such that $I:=(\alpha ; \beta), J:=(\gamma ; \alpha)$ and $I \cap J \neq \emptyset$. It is then sufficient to prove that if $A \in \mathscr{A}(I) \cap \mathscr{A}(J)$ then $\rho_{I}(A)=\rho_{J}(A)$. Without loss of generality we may also assume that $\|A\| \leq 1$.

(a) Let $\alpha_{n} \rightarrow \alpha$, for $n \rightarrow \infty$, be such that $\left\{I_{n}\right\}_{n \in \mathbb{N}}, I_{n}:=\left(\alpha_{n} ; \beta\right)$, is an increasing sequence of intervals contained in $I$. We first prove that

$$
\left\{\bigcup_{n \in \mathbb{N}}\left(\mathscr{A}\left(I_{n}\right) \cap \mathscr{A}(J)\right)\right\}^{-\omega}=\mathscr{C}(I) \cap \mathscr{A}(J) .
$$

By taking commutants on both sides and using duality, this equality is equivalent to

$$
\left\{\bigcup_{n \in \mathbb{N}}\left(\mathscr{C}\left(I_{n}\right) \cap \mathscr{A}(J)\right)\right\}^{\prime}=\mathscr{A}\left(I^{\prime}\right) \vee \mathscr{A}\left(J^{\prime}\right) \text {. }
$$

But

$$
\begin{aligned}
\left\{\bigcup_{n \in \mathbb{N}}\left(\mathscr{b}\left(I_{n}\right) \cap \mathscr{C}(J)\right)\right\}^{\prime} & =\left\{\left(\bigcup_{n \in \mathbb{N}} \mathscr{C}\left(I_{n}\right)\right) \cap \mathscr{A}(J)\right\}^{\prime} \\
& =\left(\bigcup_{n \in \mathbb{N}} \mathscr{A}\left(I_{n}\right)\right)^{\prime} \vee \mathscr{A}\left(J^{\prime}\right) \\
& =\bigcap_{n \in \mathbb{N}} \mathscr{A}\left(I_{n}^{\prime}\right) \vee \mathscr{A}\left(J^{\prime}\right) \\
& =\mathscr{A}\left(I^{\prime}\right) \vee \mathscr{A}\left(J^{\prime}\right),
\end{aligned}
$$

where the last equality follows by conformal invariance of the net [31].

(b) It now follows from (a) and Kaplanski's density theorem that there exists a sequence $\left(A_{n}\right)_{n \in \mathbb{N}}$ with $A_{n} \in \mathscr{A}\left(I_{n}\right) \cap \mathscr{A}(J),\|A\| \leq 1$ such that $\omega-\lim _{n \rightarrow \infty} A_{n}=A$. Then by weak continuity of $\rho_{I}$ on $\mathscr{A}(I)$,

$$
\rho_{I}(A)=\omega-\lim _{n \rightarrow \infty} \rho_{I}\left(A_{n}\right) .
$$

Since $I_{n} \subset I$ and $A_{n} \in \mathscr{A}\left(I_{n}\right)$,

$$
\rho_{I}\left(A_{n}\right)=\rho_{I_{n}}\left(A_{n}\right)
$$

and since $I_{n} \cup J$ is an interval with non-empty complement,

$$
\rho_{I_{n}}\left(A_{n}\right)=\rho_{I_{n} \cup J}\left(A_{n}\right)=\rho_{I_{n}}\left(A_{n}\right),
$$

where the last equality follows from $A_{n} \in \mathscr{A}\left(I_{n}\right) \cap \mathscr{C}(J)$. Hence,

$$
\rho_{I}(A)=\omega-\lim _{n \rightarrow \infty} \rho_{J}\left(A_{n}\right)=\rho_{J}(A),
$$

by weak continuity of $\rho_{J}$ on $\mathscr{C}(J)$. This completes the proof.

Let now $J \subset \mathbb{R}$ be an interval containing $I_{0 \mathbb{R}}$ and $I$. Then

$$
\begin{aligned}
& J \cup J_{>} \supseteq I_{0 \mathbb{R}} \cup I \cup I_{>}, \\
& J \cup J_{<} \supseteq I_{0 \mathbb{R}} \cup I \cup I_{<},
\end{aligned}
$$


so that by locality and duality,

$$
\begin{aligned}
& \rho_{\left(I \cup I_{<}\right)_{S^{1}}}(A)=\rho_{\left(J \cup J_{<)_{S}}\right.}(A) \in \mathscr{A}\left(\left(J \cup J_{<}\right)_{S^{1}}\right), \\
& \rho_{\left(I \cup I_{>}\right)_{S^{1}}}(A)=\rho_{\left(J \cup J_{>}\right)_{S^{1}}}(A) \in \mathscr{A}\left(\left(J \cup J_{>}\right)_{S^{1}}\right),
\end{aligned}
$$

which means that

$$
\rho_{\alpha}^{d}(A) \in \mathscr{A}\left(\left(J \cup J_{>}\right)_{S^{1}}\right) \cap \mathscr{A}\left(\left(J \cup J_{<}\right)_{S^{1}}\right)=\mathscr{C}_{\alpha}^{d}(J),
$$

and this shows that $\rho_{\alpha}^{d}$ is an endomorphism of $\overline{\mathscr{C}}_{\alpha}^{d}$.

We now check that

$$
\left.\rho_{\alpha}^{d}\right|_{\ell_{\alpha}^{d}\left(\left(I_{0 \mathbb{R}}\right)^{\prime \mathbb{R}}\right)}=\left.\mathrm{id}\right|_{\ell_{\alpha}^{d}\left(\left(I_{0 \mathbb{R}}\right)^{\prime \mathbb{R}}\right)} .
$$

Since $\mathscr{C}_{\alpha}^{d}\left(\left(I_{0 \mathbb{R}}\right)^{\prime \mathbb{R}}\right)$ is the $C^{*}$-algebra generated by the $\mathscr{b}_{\alpha}^{d}(J)$ with $J \subset\left(I_{0 \mathbb{R}}\right)^{\prime} \mathbb{R}$ and $\rho_{\alpha}^{d}$ is norm-continuous, it is sufficient to check that $\left.\rho_{\alpha}^{d}\right|_{\ell_{\alpha}^{d}(J)}=\left.\mathrm{id}\right|_{\ell_{\alpha}^{d}(J)}$, for $J \subset\left(I_{0 \mathbb{R}}\right)^{\prime \mathbb{R}}$. We may assume that $J$ lies on the left of $I_{0 \mathbb{R}}$ so that $\left(J_{<} \cup J\right) \cap I_{0 \mathbb{R}}=\emptyset$. Hence for $A \in \mathscr{A}_{\alpha}^{d}(J)$,

$$
\rho_{\alpha}^{d}(A)=\rho_{\left(J \cup J_{<S^{1}}\right.}(A)=A,
$$

since $\rho$ is localized in $I_{0}$ and $\left(J \cup J_{<}\right)_{S^{1}} \cap I_{0}=\emptyset$. This completes the proof of (ii).

(iii) The covariance of $\rho_{\alpha}$ and $\rho_{\alpha}^{d}$ is an immediate consequence of the Moebius covariance of $\rho$ and the fact that we restrict our attention to Moebius transformations leaving $\alpha$ fixed and which, consequently, leave $\mathscr{b}_{\alpha}$ and $\mathscr{f}_{\alpha}^{d}$ invariant.

Remarks 4.14. (i) From now on, when we consider representations of $\mathscr{b}_{0}$ localized in a given interval $I_{0}$ we will call $\alpha \in S^{1} \backslash I_{0}^{-}$a point at infinity.

(ii) Let $\rho_{1}$ and $\rho_{2}$ be representations of $\mathscr{A}_{0}$ localized in $I_{0}$, and $\alpha$ a point at infinity. Since $\rho_{i \alpha}$ (resp. $\left.\rho_{i \alpha}^{d}\right)$ are endomorphisms of $\overline{\mathscr{b}}_{\alpha}\left(\right.$ resp. $\overline{\mathscr{C}}_{\alpha}^{d}$ ) for $i=1,2$, they can be composed. It is immediate that the composition of $\rho_{1}$ and of $\rho_{2}$ as representations of $\mathscr{A}_{0}$ coincides with $\rho_{1 \alpha} \circ \rho_{2 \alpha}$ on $\mathscr{A}_{\alpha}$ (resp. with $\rho_{1 \alpha}^{d} \circ \rho_{2 \alpha}^{d}$ on $\mathscr{A}_{\alpha}^{d}$ ) that is, $\left(\rho_{1} \hat{\circ} \rho_{2}\right)_{\alpha}=\rho_{1 \alpha} \circ \rho_{2 \alpha}$ (resp. $\left.\left(\rho_{1} \hat{\circ} \rho_{2}\right)_{\alpha}^{d}=\rho_{1 \alpha}^{d} \circ \rho_{2 \alpha}^{d}\right)$.

(iii) In view of the preceding remarks, we shall usually not distinguish $\rho$ from $\rho_{\alpha}$ and $\rho_{\alpha}^{d}$ and write $\rho$ for $\rho_{\alpha}$ or $\rho_{\alpha}^{d}$ in the following.

\section{IV.4. Braid Statistics Operators}

We now proceed to define the notion of statistics of superselection sectors for field theories on the circle. ${ }^{7}$ Statistics operators were first defined by Doplicher, Haag and Roberts (see, e.g., [13, 14]) for local charges of theories in four dimensional space-time; their results were later generalized by Buchholz and Fredenhagen [15] to "topological" charges. It was recently observed that for two-dimensional local theories [22] and for three-dimensional theories with "topological" charges [23-25] the statistics of superselection sectors is more general, involving braid statistics. The situation considered here is analogous to the one of refs. [23-25] (see also ref. [30] for a treatment of abelian braid statistics in the example of $U(1)$-current algebra).

\footnotetext{
7 In this section we present a short summary of basic facts concerning braid statistics. For more details, see $[22,25,30]$
} 
Definition 4.15. Let $\alpha \in S^{1}$ and $p_{\alpha}$ be the stereographic projection mapping $S^{1} \backslash\{\alpha\}$ onto $\mathbb{R}$, as in the last section. If $I_{1}, I_{2}$ are two intervals on $S^{1}$ such that $I_{1} \cap I_{2}=\emptyset$ and which are mapped by $p_{\alpha}$ onto bounded intervals on $\mathbb{R}$, we write $I_{1}<_{\alpha} I_{2}$ (resp. $\left.I_{1 \alpha}>I_{2}\right)$ if $I_{1 \mathbb{R}}=p_{\alpha}\left(I_{1}\right)$ lies on the left of $I_{2 \mathbb{R}}=p_{\alpha}\left(I_{2}\right)$ (resp. $I_{1 \mathbb{R}}$ lies on the right of $\left.I_{2 \mathbb{R}}\right)$.

Definition 4.16. Let $\rho_{1}$ and $\rho_{2}$ be two representations of $\mathscr{A}_{0}$ localized in an interval $I_{0}$ and $\alpha \in S^{1}$ a point at infinity. Pick a representation $\tilde{\rho}_{1}$ (resp. $\hat{\rho}_{1}$ ) unitarily equivalent to $\rho_{1}$ and localized in an interval $\tilde{I}_{1}$ (resp. $\hat{I}_{1}$ ) such that $I_{0} \cap \tilde{I}_{1}=\emptyset, \alpha \notin \tilde{I}_{1}^{-}, \tilde{I}_{1 \alpha}>I_{0}$ (resp. $I_{0} \cap \hat{I}_{1}=\emptyset, \alpha \notin \hat{I}_{1}^{-}, \hat{I}_{1}<_{\alpha} I_{0}$ ) and let $J$ (resp. $\tilde{J}$ ) be an interval containing $I_{0} \cup \tilde{I}_{1}$ (resp. $I_{0} \cup \hat{I}_{1}$ ). If $\Gamma_{\rho_{1} \tilde{\rho}_{1}}\left(\right.$ resp. $\Gamma_{\rho_{1} \hat{\rho}_{1}}$ ) intertwines $\rho_{1}$ and $\tilde{\rho}_{1}$ (resp. $\rho_{1}$ and $\hat{\rho}_{1}$ ), define

$$
\begin{aligned}
& \varepsilon_{\rho_{1} \hat{o} \rho_{2}}^{+}:=\Gamma_{\rho_{1} \tilde{\rho}_{1}} \rho_{2, J}\left(\Gamma_{\rho_{1} \tilde{\rho}_{1}}^{*}\right) \in \mathscr{A}(J), \\
& \varepsilon_{\rho_{1} \hat{o} \rho_{2}}^{-}:=\Gamma_{\rho_{1} \hat{\rho}_{1}} \rho_{2, \tilde{J}}\left(\Gamma_{\rho_{1} \hat{\rho}_{1}}^{*}\right) \in \mathscr{A}(\tilde{J}) .
\end{aligned}
$$

Remark 4.17. Clearly, $\varepsilon_{\rho_{1} \hat{o} \rho_{2}}^{+}$and $\varepsilon_{\rho_{1} \hat{o} \rho_{2}}^{--}$do not depend on the particular choice of the point $\alpha$ at infinity made in Definition 4.16 as long as the conditions specified in Definition 4.16 hold. (The next figure shows two equivalent choices, $\alpha, \alpha^{\prime}$, of points at infinity, for $I_{0}, \tilde{I}_{1}, \hat{I}_{1}$, fixed.)

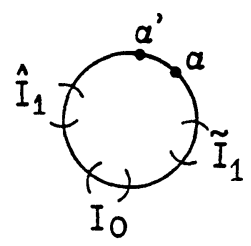

Lemma 4.18. (i) The definition of $\varepsilon_{\rho_{1} \hat{\mathrm{o}} \rho_{2}}^{+}\left(\right.$resp. $\left.\varepsilon_{\rho_{1} \hat{\mathrm{o}} \rho_{2}}^{-}\right)$is independent of the particular choice of $\tilde{I}_{1 \alpha}>I_{0}$ and $\tilde{\rho}_{1}$ (resp. of $\hat{I}_{1}<_{\alpha} I_{0}$ and $\hat{\rho}_{1}$ ) as long as they satisfy the conditions specified in Definition 4.16.

(ii) The unitary operators $\varepsilon_{\rho_{1} \hat{\circ} \rho_{2}}^{ \pm}$intertwine the representations $\rho_{1} \hat{\circ} \rho_{2}$ and $\rho_{2} \hat{\circ} \rho_{1}$,

$$
\rho_{1} \hat{o} \rho_{2}(A) \varepsilon_{\rho_{1} \hat{o} \rho_{2}}^{ \pm}=\varepsilon_{\rho_{1} \hat{o} \rho_{2}}^{ \pm} \rho_{2} \hat{o} \rho_{1}(A) \quad \forall A \in \mathscr{A}_{0}
$$

and satisfy

$$
\varepsilon_{\rho_{1} \hat{o} \rho_{2}}^{+} \cdot \varepsilon_{\rho_{1} \hat{o} \rho_{2}}^{-}=\mathbb{1}_{\mathscr{H}} \cdot
$$

Proof. See, for example, refs. [13, 22-25 or 30] for the proof of (i) and of Eq. (4.7). Equation (4.6) is an immediate consequence of Lemma 4.8.

If $\rho$ is a representation of $\mathscr{C}_{0}$ localized in some interval, we write $\rho^{\hat{\imath} \imath}$ for the composed representation $\rho \hat{o} \ldots \hat{o} \rho$ ( $i$-times).

Definition 4.19. Let $B_{n}$ denote the braid group on $n$ strands with generators $\sigma_{1}, \ldots \sigma_{n-1}$ and relations

$$
\begin{aligned}
\sigma_{i} \sigma_{\imath+1} \sigma_{\imath} & =\sigma_{i+1} \sigma_{i} \sigma_{i+1}, & i & =1, \ldots n-2 \\
\sigma_{i} \sigma_{j} & =\sigma_{j} \sigma_{i} \quad \text { if }|i-j|>1, & i, j & =1, \ldots n-1
\end{aligned}
$$


If $\rho$ is a representation of $\mathscr{C}_{0}$ localized in $I_{0}$ we define

$$
\pi_{n}^{\rho}\left(\sigma_{i}\right):=\rho^{\hat{o} i-1}\left(\varepsilon_{\rho \hat{o} \rho}^{+}\right), \quad i=1, \ldots n-1 .
$$

Theorem 4.20. (i) The map $\pi_{n}^{\rho}: B_{n} \rightarrow \rho^{\hat{o} n}\left(\mathscr{A}_{0}\right)^{\prime}$ extends to a unitary representation of the braid group on $n$ strands $B_{n}$.

(ii) This representation depends, up to unitary equivalence, only on the sector $[\rho]$ of $\rho$.

Proof. To prove (i), it is sufficient to check the relations (4.8) and (4.9) for the operators defined by Eq. (4.10), see refs. [23-25 or 22]. Part (ii) follows from Remark 4.17 and arguments analogous to the one used in ref. [13], Theorem 4.3.

\section{IV.5. Superselection Sectors with Finite Statistical Dimension}

We now show that several classical results on the superselection structure of sectors having finite statistical dimension in theories defined on Minkowski space-time also hold for conformal field theories on the circle. Our strategy is the following. We choose a point at infinity $\alpha \in S^{1}$ and apply well-known theorems of Doplicher, Haag and Roberts (as adapted in ref. [22] for low-dimensional theories) to the nets defined in Sect. IV.3. We subsequently show that all the results obtained in this way are independent of the particular choice of the point at infinity. Clearly, this way of proceeding is not very natural in the present context. It has however the advantage of considerably reducing further technical work.

We start with the following lemma.

Lemma 4.21. Let $\rho$ be a covariant representation of $\mathscr{C}_{0}$ localized in the intervals $I$ and $J \subseteq S^{1}$; let $\tilde{\rho}$ be unitarily equivalent to $\rho$ and localized in $I$. Then

$$
\begin{aligned}
\rho(\mathscr{A}(I))^{\prime} \cap \mathscr{C}(I) & \cong \rho(\mathscr{A}(J))^{\prime} \cap \mathscr{A}(J), \\
\rho(A(I))^{\prime} \cap \mathscr{C}(I) & \cong \tilde{\rho}(\mathscr{C}(I))^{\prime} \cap \mathscr{A}(I) .
\end{aligned}
$$

Proof. Ad (ii). Let $U$ be a unitary operator intertwining $\rho$ and $\tilde{\rho}$,

$$
\rho(A) U=U \tilde{\rho}(A), \quad \forall A \in A_{0} .
$$

By locality and duality, $U \in \mathscr{A}(I)$ so that

$$
\begin{aligned}
\tilde{\rho}(\mathscr{A}(I))^{\prime} \cap \mathscr{A}(I) & \cong U\left\{\tilde{\rho}(\mathscr{A}(I))^{\prime} \cap \mathscr{A}(I)\right\} U^{*} \\
& =U \tilde{\rho}(\mathscr{A}(I))^{\prime} U^{*} \cap U \mathscr{b}(I) U^{*} \\
& =\left(U \tilde{\rho}(\mathscr{A}(I)) U^{*}\right)^{\prime} \cap \mathscr{A}(I) \\
& =\rho(\mathscr{A}(I))^{\prime} \cap \mathscr{A}(I) .
\end{aligned}
$$

Ad (i). Choose $g \in P S U(1 ; 1)$ such that $g \cdot I=J$. Then

$$
\begin{aligned}
\rho(\mathscr{b}(J))^{\prime} \cap \mathscr{C}(J) & \cong \pi_{0}^{M}(g)\left(\rho(\mathscr{C}(J))^{\prime} \cap \mathscr{b}(J)\right) \pi_{0}^{M}(g)^{*}, \\
& =\left\{\pi_{0}^{M}(g) \rho(\mathscr{A}(J))^{\prime} \pi_{0}^{M}(g)^{*}\right\} \cap \pi_{0}^{M}(g) \mathscr{A}(J) \pi_{0}^{M}(g)^{*}, \\
& =\rho_{g}\left(\alpha_{g}(\mathscr{C}(J))\right)^{\prime} \cap \mathscr{C}(I) \\
& =\rho_{g}(\mathscr{b}(I))^{\prime} \cap \mathscr{A}(I) .
\end{aligned}
$$

But $\rho_{g}$ is unitarily equivalent to $\rho$ and localized in $I$, so that the conclusion follows from (ii). 
Definition 4.22. Let $[\pi]$ be a sector of $\mathscr{A}_{0}$. Pick $\rho \in[\pi]$ localized in $I \subset S^{1}$. The sector $[\pi]$ is said to be locally irreducible if

$$
\rho(\mathscr{A}(I))^{\prime} \cap \mathscr{C}(I)=\mathbb{C} \cdot \mathbb{1}_{\mathscr{H}} .
$$

Remark 4.23. (i) It follows from Lemma 4.21 that Eq. (4.11) is independent of the particular choices of $\rho \in[\pi]$ and of $I \subset S^{1}$.

(ii) Let $\rho \in[\pi]$ be localized in $I$ and $\alpha \in S^{1}$ a point at infinity. Since

$$
\rho\left(\mathscr{C}_{0}\right)^{\prime} \subseteq \rho\left(\overline{\mathscr{A}}_{\alpha}\right)^{\prime} \subseteq \rho(\mathscr{A}(I))^{\prime} \cap \mathscr{A}(I)
$$

Eq. (4.11) implies that the sector $[\pi]$ is irreducible.

Given a $C^{*}$-algebra $\mathscr{A}$ acting on a Hilbert space $\mathscr{H}$, let $\mathscr{N}^{\cdot / b}$ denote the set of bounded linear mappings from $\mathscr{C}$ into $\mathscr{B}(\mathscr{H})$ equipped with the point weak open topology (see ref. [13]). If $\phi \in \mathscr{C}^{\not h}$, we define the norm of $\phi$ as usual,

$$
\|\phi\|:=\sup _{A \in \mathscr{\ell},} \frac{\|\phi(A)\|}{\|A\|}
$$

and the unit ball

$$
\mathscr{H C}_{1}^{\prime \prime}:=\left\{\phi \in \mathscr{M}^{\mathcal{L}} \mid\|\phi\| \leq 1\right\}
$$

is a compact subset of $\mathscr{B} 6^{\mathscr{C}}$ in the point weak open topology.

Let $\rho$ be a representation of $\mathscr{C}_{0}$ localized in the interval $I$; choose a point at infinity $\alpha \in S^{1}$, and let $I_{\mathbb{R}}=p_{\alpha}(I)$, where $p_{\alpha}: S^{1} \backslash\{\alpha\} \rightarrow \mathbb{R}$ is the stereographic projection already considered in Sect. IV.3. In the following we do not distinguish between $\rho$ and $\rho_{\alpha}^{d}$. Note that $\rho_{\alpha}^{d}$ is localized in $I_{\mathbb{R}}$.

Definition 4.24. A positive linear mapping $\phi \in \mathscr{M}^{-\bar{l}_{\alpha}^{d}}$ is called a left inverse for $\rho$ on $\bar{A}_{\alpha}^{d}$ if

$$
\begin{aligned}
\phi(A \rho(B)) & =\phi(A) B \quad \forall A, B \in \overline{\mathscr{b}}_{\alpha}^{d}, \\
\phi\left(\mathbb{1}_{\mathscr{H}}\right) & =\mathbb{1}_{\mathscr{H}} .
\end{aligned}
$$

Remark 4.25. (i) Since a positive mapping is automatically self-adjoint, we also have that

$$
\phi(\rho(A) B)=A \phi(B) \quad \forall A, B \in \bar{\phi}_{\alpha}^{d} .
$$

(ii) If $\rho$ is localized in $I$ then

$$
\left.\phi\right|_{\ell_{\alpha}^{d}\left(\left(I_{\mathbb{R}}\right)^{\prime} \mathbb{R}\right)}=\left.\mathrm{id}\right|_{\ell_{\alpha}^{d}\left(\left(I_{\mathbb{R}}\right)^{\prime \mathbb{R}}\right)} .
$$

(iii) Since the net $\overline{\mathscr{C}}_{\alpha}^{d}$ is dual on $\mathbb{R}$, for any interval $J \supseteq I_{\mathbb{R}}$,

$$
\phi\left(\mathscr{C}_{\alpha}^{d}(J)\right) \subseteq \cdot \mathscr{A}_{\alpha}^{d}(J)
$$

so that $\phi$ maps. $\bar{b}_{\alpha}^{d}$ into $\overline{\mathscr{b}}_{\alpha}^{d}$.

We now pick a sequence of bounded intervals $\left\{I_{k}\right\}_{k \in \mathbb{N}}, I_{k} \subseteq \mathbb{R}$, "converging" toward infinity in the following sense. For any bounded interval $I \subseteq \mathbb{R}$, there exists $k_{0}(I) \in \mathbb{N}$ such that

$$
I \cap I_{k}=\emptyset \quad \forall k \geq k_{0}(I) \text {. }
$$


If $\rho_{k}$ is a representation of $\mathscr{A}_{0}$ localized in $I_{k S^{1}}$ and $U_{k}$ a unitary operator intertwining $\rho$ and $\rho_{k}$,

$$
\rho_{k}(A) U_{k}=U_{k} \rho(A) \quad \forall A \in \mathscr{C}_{0}
$$

then

$$
\rho(A)=\lim _{k \rightarrow \infty} U_{k}^{*} A U_{k} \quad \forall A \in \overline{\mathscr{b}}_{\alpha}^{d},
$$

where convergence is understood in the norm topology.

Lemma 4.26. Let $U_{k}, k \in \mathbb{N}$, be defined as above. Then the sequence of maps $\left\{\operatorname{ad} U_{k}\right\}_{k \in \mathbb{N}} \subseteq \mathscr{C}_{1}^{\tilde{H}_{\alpha}^{d}}$ has at least one limit point and every such limit point is a left inverse for $\rho$ on $\overline{\mathscr{A}}_{\alpha}^{d}$.

Proof. See ref. [13], Chap. III

Lemma 4.27. The set of all left inverses of a given endomorphism $\rho$ on $\bar{A}_{\alpha}^{d}$ is a non-void, compact convex subset of the unit ball $\mathscr{M G}_{1}^{\pi_{\alpha}^{d}}$.

Proof. See ref. [13], Chap. III.

Lemma 4.28. Let $\rho$ be locally irreducible, $\varepsilon_{\rho \hat{o} \rho}^{ \pm}$be as in Definition 4.16 and $\phi$ a left inverse for $\rho$ on $\bar{A}_{\alpha}^{d}$. Then

(i) $\phi\left(\varepsilon_{\rho \overline{\mathrm{o}} \rho}^{+}\right)=\lambda \cdot \mathbb{1}_{\mathscr{H}}$, for some $\lambda \in \mathbb{C}$.

(ii) $\phi\left(\varepsilon_{\rho \overline{0} \rho}^{-}\right)=\bar{\lambda} \cdot \mathbb{1}_{\mathscr{H}},(\lambda$ defined by $(i))$.

(iii) If $\phi_{0}$ is a left inverse for $\rho$ obtained by the limiting procedure of Lemma 4.26, then

$$
\begin{aligned}
\left\|\phi\left(A^{*} A\right)\right\| & \geq|\lambda|^{2}\left\|A^{*} A\right\|, \\
\phi\left(A^{*} A\right) & \geq|\lambda|^{2} \phi_{0}\left(A^{*} A\right),
\end{aligned}
$$

where $\lambda$ is defined by (i).

Proof. Ad (i). Pick $A \in \overline{\mathscr{b}}_{\alpha} \subseteq \overline{\mathscr{b}}_{\alpha}^{d}$, then

$$
\begin{aligned}
\phi\left(\varepsilon_{\rho \hat{o} \rho}^{+}\right) \rho(A) & =\phi\left(\varepsilon_{\rho \hat{o} \rho}^{+} \rho^{2}(A)\right) \\
& =\phi\left(\varepsilon_{\rho \hat{o} \rho}^{+} \rho \hat{o} \rho(A)\right) \\
& =\phi\left(\rho \hat{o} \rho(A) \varepsilon_{\rho \hat{o} \rho}^{+}\right)=\rho(A) \phi\left(\varepsilon_{\rho \hat{o} \rho}^{+}\right)
\end{aligned}
$$

so that $\phi\left(\varepsilon_{\rho \hat{o} \rho}^{+}\right) \in \rho\left(\bar{t}_{\alpha}\right)^{\prime} \subseteq \rho(\mathscr{A}(I))^{\prime} \cap \mathscr{A}(I)=\mathbb{C} \cdot \mathbb{1}_{\mathscr{H}}$, by the local irreducibility of $\rho$. This completes the proof of (i). Part (ii) follows from Remark 4.25 (i) and Eq. (4.7). For the proof of (iii), see ref. [13], Chap. III.

Definition 4.29. Let $\rho$ be a locally irreducible representation of $A_{0}$ localized in $I$ and $\alpha$ a point at infinity. We say that $\rho$ has finite statistical dimension if there exists a left inverse $\phi$ for $\rho$ on $\overline{\mathscr{A}}_{\alpha}^{d}$ such that $\phi\left(\varepsilon_{\rho \hat{o} \rho}^{+}\right)=\lambda \cdot \mathbb{1}_{\mathscr{H}}$ with $\lambda \neq 0$. The statistical dimension of $\rho$ is then defined by

$$
d(\rho):=|\lambda|^{-1}
$$

Theorem 4.30. (i) If $\rho$ is locally irreducible with finite statistical dimension then $\rho$ has, a unique left inverse $\phi$ on $\bar{\ell}_{\alpha}^{d}$.

(ii) The sequence of charge transport operators $\left\{\operatorname{ad} U_{k}\right\}_{k \in \mathbb{N}}$ defined in Eq. (4.14) converges to $\phi$ in $\mathscr{M}_{1}^{\bar{\lambda}_{\alpha}^{d}}$. 
(iii) If $\tilde{\rho} \in[p]$ is a representation of. Ao localized in an interval $J$ such that $\alpha$ is a point at infinity for $\tilde{\rho}$, then $\tilde{\rho}$, too, has a unique left inverse $\tilde{\phi}$ on $\bar{\phi}_{\alpha}^{d}$ and

$$
\phi\left(\varepsilon_{\rho \hat{o} \rho}^{+}\right)=\tilde{\phi}\left(\varepsilon_{\tilde{\rho} \hat{o} \tilde{\rho}}^{+}\right) .
$$

Proof. See ref. [13], Chap. III and ref. [22].

We now establish that the statistical dimension is characteristic of the locally irreducible sector $[\rho]$ by showing that its definition is independent of the particular point at infinity,

Theorem 4.31. Let $\rho$ be a locally irreducible representation of $\mathscr{A}_{0}$ localized in $I \subset S^{1}$ and $\alpha, \beta$ be two possible points at infinity. If $\rho$ has finite statistical dimension and $\phi_{\alpha}$ (resp. $\phi_{\beta}$ ) is the left inverse of $\rho$ on $\overline{\mathscr{b}}_{\alpha}^{d}$ (resp. on $\overline{\mathscr{C}}_{\beta}^{d}$ ) then

$$
\left.\phi_{\alpha}\right|_{\mathscr{\ell}(J)}=\left.\phi_{\beta}\right|_{\mathscr{\ell}(J)}
$$

for any interval $J \ni I$ such that $\alpha, \beta \notin J^{-}$. In particular

$$
\phi_{\alpha}\left(\varepsilon_{\rho \hat{o} \rho}^{+}\right)=\phi_{\beta}\left(\varepsilon_{\rho \hat{o} \rho}^{+}\right)
$$

so that $d(\rho)$ is an invariant of the sector $[\rho]$.

For the proof of Theorem 4.31 we use the following concepts.

Definition 4.32. (i) Given an interval $I \subset S^{1}$, let $\mathscr{b}_{I}$ be the $C^{*}$-inductive limit of

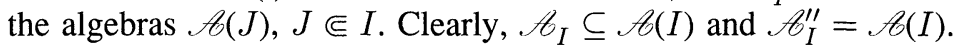

(ii) Let $\rho$ be a locally irreducible representation of $\mathscr{C}_{0}$ localized in $I$, and $J$ an interval such that $J \ni I$. A local left inverse for $\rho$ on $\mathscr{A}_{J}$ is a positive linear mapping $\phi_{J} \in \mathscr{C}^{\mathscr{C}_{J}}$ satisfying Eqs. (4.12), (4.13), for $A, B \in \mathscr{C}_{J}$, and such that

$$
\phi\left(\varepsilon_{\rho \hat{o} \rho}^{+}\right)=\lambda \cdot \mathbb{1}, \quad \lambda \in \mathbb{C} .
$$

Proof of Theorem 4.31. Since $\rho$ has finite statistical dimension, the unique left inverse $\phi_{\alpha}$ for $\rho$ on $\bar{t}_{\alpha}^{d}$ satisfies $\phi_{\alpha}\left(\varepsilon_{\rho \hat{o} \rho}^{+}\right)=\lambda \cdot \mathbb{1} \neq 0$. Let $J$ be an interval such that $J \ni I, \alpha \notin J^{-}$. The restriction of $\phi_{\alpha}$ to $\mathscr{A}_{J}$ is a local left inverse for $\rho$ on $\mathscr{A}_{J}$. If $J=(\gamma, \delta)$, we pick a sequence of intervals $\left\{J_{k}\right\}_{k \in \mathbb{N}}$ on $S^{1}$ such that $J_{k} \subseteq J$ and which "converges towards $\delta$ " in the following sense. If $\tilde{J}$ is any interval on $S^{1}$ for which $\delta \notin \tilde{J}^{-}$then there exists $k_{0}(\tilde{J})$ such that

$$
\tilde{J} \cap J_{k}=\emptyset \quad \forall k \geq k_{0}(\tilde{J}) .
$$

For $k \in \mathbb{N}$, pick a representation $\rho_{k}$ localized in $J_{k}$ unitarily equivalent to $\rho$ and $V_{k}$ intertwining $\rho$ and $\rho_{k}$,

$$
\rho_{k}(A) V_{k}=V_{k} \rho(A) \quad \forall A \in \mathscr{\ell}_{0} .
$$

Just as in Lemma 4.26, any weak limit point $\phi_{\delta}$ of $\left\{\operatorname{ad} V_{k}\right\}_{k \in \mathbb{N}}$ in $\mathscr{M}_{1}^{\bar{\ell}_{\delta}^{d}}$ is a left inverse of $\rho$ on $\bar{\ell}_{\delta}^{d}$ and, as in Lemma 4.28 (i), it has the property that $\phi_{\delta}\left(\varepsilon_{\rho \hat{o} \rho}^{+}\right)=\mu \cdot \mathbb{1}$, $\mu \in \mathbb{C}$, since $\rho$ is locally irreducible. Hence the restriction of $\phi_{\delta}$ to $\mathscr{A}_{J}$ is a local left inverse for $\rho$ on $\mathscr{A}_{J}$. Furthermore, the proof of Lemma 4.28 (iii), as given in ref. [13], can be repeated, word by word, to show that if $\phi$ is any local left inverse for $\rho$ on $A_{J}$ and $\phi\left(\varepsilon_{\rho \hat{o} \rho}^{+}\right)=\eta \cdot \mathbb{1}, \eta \in \mathbb{C}$, then

$$
\phi\left(A^{*} A\right) \geq|\eta|^{2} \phi_{\delta}\left(A^{*} A\right) \quad \forall A \in \mathscr{C}_{J} .
$$


We now proceed as in ref. [13]: the set of local left inverses for $\rho$ on $\mathscr{C}_{J}$ is a compact convex subset of $\mathscr{O b}_{1}^{\mathfrak{b}_{J}}$, since Eq. (4.15) is also preserved by convex combinations and taking limits. Hence, by the Krein-Milman theorem, there exists an extremal local left inverse $\phi_{\text {ext }}$ such that $\phi_{\text {ext }}\left(\varepsilon_{\rho \hat{o} \rho}^{+}\right)=\eta \cdot \mathbb{1} \neq 0$, because $\phi_{\alpha}\left(\varepsilon_{\rho \hat{o} \rho}^{+}\right)=\lambda \cdot \mathbb{1} \neq 0$. But

$$
\phi_{\mathrm{ext}}=\left(1-\xi^{2}\right) \phi_{1}+\xi^{2} \phi_{\delta}, \quad \xi \in \mathbb{R}, \quad 0<\xi^{2}<|\eta|^{2},
$$

where $\phi_{1}:=\frac{\phi_{\text {ext }}-\xi^{2} \phi_{\delta}}{1-\xi^{2}}$ is still a local left inverse for an $\mathscr{C}_{J}$ since by Eq. (4.17) it is still a positive map. Equation (4.18) contradicts the extremality of $\phi_{\text {ext }}$ unless $\phi_{\text {ext }} \equiv \phi_{\delta}$. In particular, $\phi_{\delta}\left(\varepsilon_{\rho \hat{o} \rho}^{+}\right)=\mu \cdot \mathbb{1} \neq 0$, and $\phi_{\text {ext }}$ is the unique extremal local left inverse on $\mathscr{C}_{J}$ with finite statistical dimension. Repeating the same construction for the second endpoint $\gamma$ of $J$ we see that $\left.\phi_{\gamma}\right|_{\mathscr{B}_{J}}=\phi_{\text {ext }}=\left.\phi_{\delta}\right|_{\mathscr{C}_{J} J}$. In particular $\left.\phi_{\gamma}\right|_{\mathscr{B}(\tilde{J})}=\left.\phi_{\delta}\right|_{\mathscr{B}(\tilde{J})}$ for any interval $\tilde{J} \Subset J$. Letting the endpoints $\gamma$ and $\delta$ of $J$ vary we obtain the statement of the theorem.

Corollary 4.33. Let $\rho$ be a locally irreducible representation of $\mathscr{b}_{0}$ localized in the interval $I \subset S^{1}$, and having finite statistical dimension. Let $\alpha \in S^{1}$ be a point at infinity and $J \ni I$ an interval such that $\alpha \notin J^{-}$. The left inverse $\phi_{\alpha}$ of $\rho$ on $\bar{\phi}_{\alpha}^{d}$ has the property that

$$
\phi(\mathscr{A}(J)) \subseteq \mathscr{A}(J) .
$$

Proof. Let $J_{0}=(\gamma, \delta)$ be any interval such that $J_{0} \ni J$. Choose a sequence of interval $\left\{J_{k}\right\}_{k \in \mathbb{N}}, J_{k} \subseteq J_{0}$, "converging towards $\delta$ " as in the proof of Theorem 4.31, representations $\rho_{k}$ localized in $J_{k}$ and interwiners $V_{k}$ satisfying Eq. (4.16). Clearly $V_{k} \in \mathscr{A}\left(J_{0}\right)$, so that if $A \in \mathscr{C}(J)$ then $\phi_{\alpha}(A) \in \mathscr{C}\left(J_{0}\right)$, since it is a weak limit point of $V_{k} A V_{k}^{*}$. This means that

$$
\phi(\mathscr{A}(J)) \subseteq \bigcap_{J_{0} \ni J} \mathscr{A}\left(J_{0}\right)
$$

Because of the conformal invariance of the net [31],

$$
\mathscr{A}(J)=\bigcap_{J_{0} \ni J} \mathscr{C}\left(J_{0}\right) .
$$

This completes the proof of the corollary.

The following theorem, due to Longo [67], is also valid in the present situation; (see also ref. [22], Eq. (4.40), and ref. [25], Eq. (6.38), for related results).

Theorem 4.34. Let $\rho$ be a locally irreducible representation of $\mathscr{A}_{0}$ with finite statistics localized in $I$ and $\alpha$ a point at infinity. Then

$$
\operatorname{Ind}[\rho(\mathscr{A}(I)): \mathscr{A}(I)]=d(\rho)^{2},
$$

where Ind[ : ·] denotes the minimal index of the inclusion $\rho(\mathscr{b}(I)) \subseteq \mathscr{A}(I)$.

Proof. It follows from Corollary 4.33 that $\varepsilon:=\rho \circ \phi$ is a conditional expectation of $\mathscr{b}(I)$ onto $\rho(\mathscr{b}(I))$. Since $\rho$ is a localized endomorphism of $\overline{\mathscr{b}}_{\alpha}^{d}$, we construct a localized endomorphism of $\overline{\mathscr{b}}_{\alpha}^{d}$ conjugate to $\rho$ just as in ref. [22], Appendix B. The proof of Theorem 8.5 in ref. [67] now shows that

$$
d(\rho)^{2} \operatorname{Ind}_{\varepsilon}[\rho(\mathscr{A}(I)): \mathcal{A}(I)],
$$


where $\operatorname{Ind}_{\varepsilon}[\cdot: \cdot]$ is the index associated to the conditional expectation $\varepsilon$. Since $\rho$ is locally irreducible, $\operatorname{Ind}_{\varepsilon}[\cdot: \cdot]$ coincides with the minimal index [67] and the proof is complete.

Since the set of (locally) irreducible representations of $\mathscr{b}_{0}$ is not closed under composition, we must generalize the previous considerations. At this point we need to assume that the superselection structure of a conformal net $\mathscr{A}_{0}$ can be determined by a single local algebra.

Assumption 4.35. Let $\rho, \rho^{\prime}$ be representations of $\mathscr{\ell}_{0}$ localized in $I$. Suppose that $S \in . \mathscr{C}(I)$ and that

$$
S \rho_{I}(A)=\rho_{I}^{\prime}(A) S, \quad \forall A \in \mathscr{A}(I) .
$$

Then $S$ intertwines $\rho$ and $\rho^{\prime}$, i.e.,

$$
S \rho(A)=\rho^{\prime}(A) S, \quad \forall A \in \mathscr{A}_{0} .
$$

Remark 4.36. (i) J. Roberts has shown that this assumption holds for dilatationinvariant theories on Minkowski space-times of dimension greater or equal to three (see ref. [66], Theorem 4.3). However, the proof given in [66] does not apply directly to the present situation.

(ii) Equation (4.19) implies that, for $\rho$ localized in $I$,

$$
\rho(\mathscr{A}(I))^{\prime} \cap \mathscr{A}(I)=\rho\left(\mathscr{A}_{0}\right)^{\prime}
$$

so that, in particular, irreducibility (Definition 4.3 (i)) and local irreducibility (Definition 4.22) coincide.

Definition 4.37. Let $\rho$ be a representation of $\mathscr{\theta}_{0}$ localized in $I$ and $\alpha \in S^{1}$ a point at infinity.

(i) A left inverse $\phi$ for $\rho$ on $\overline{\mathscr{b}}_{\alpha}^{d}$ is said to be standard if

$$
\phi\left(\varepsilon_{\rho \hat{\mathrm{o} \rho} \rho}^{+}\right) \phi\left(\varepsilon_{\rho \hat{\mathrm{o} \rho} \rho}^{+}\right)^{*}=\phi\left(\varepsilon_{\rho \hat{\mathrm{o}} \rho}^{+}\right)^{*} \phi\left(\varepsilon_{\rho \hat{\mathrm{o} \rho} \rho}^{+}\right)=\xi \cdot \mathbb{1}_{\mathscr{H}}, \quad \xi \in \mathbb{C} .
$$

(ii) The representation $\rho$ is said to have finite statistical dimension if there exists a standard left inverse of $\rho$ on $\overline{\mathscr{C}}_{\alpha}^{d}$ such that $\phi\left(\varepsilon_{\rho \hat{o} \rho}^{+}\right)^{*} \phi\left(\varepsilon_{\rho \hat{o} \rho}^{+}\right)=\xi \cdot \mathbb{1}_{\mathscr{H}} \neq 0$. The statistical dimension of $\rho$ is then defined by

$$
d(\rho)=|\xi|^{-1 / 2}
$$

The following results are adapted from the usual setting to the present situation without essential modifications.

Theorem 4.38. (i) Let $\rho$ be a representation of $\mathscr{b}_{0}$ having finite statistical dimension. Then $\rho$ is a finite direct sum of irreducible representations of $\mathscr{A}_{0}$ having finite statistical dimension,

$$
\rho \cong \bigoplus_{i=1}^{m} \rho_{i}, \quad d\left(\rho_{i}\right)<\infty \quad \forall i=1, \ldots m
$$

and $d(\rho)=\sum_{i=1}^{m} d\left(\rho_{i}\right)$ 
(ii) If $\rho_{1}$ and $\rho_{2}$ are representations of $\mathscr{A}_{0}$ having finite statistical dimension then $\rho_{1} \hat{o} \rho_{2}$ has finite statistical dimension and

$$
d\left(\rho_{1} \hat{\circ} \rho_{2}\right)=d\left(\rho_{1}\right) \cdot d\left(\rho_{2}\right) .
$$

Proof. (i) Since $\rho\left(\mathscr{C}_{0}\right)^{\prime} \subseteq \rho\left(\overline{\mathscr{b}}_{\alpha}^{d}\right)^{\prime}$, the proof that $\rho$ is a finite direct sum of irreducible representations of $\mathscr{A}_{0}$ is just as in ref. [13], Lemma 6.1. Covariance of the subrepresentations then follows by the proof given in ref. [14], Lemma 2.2. The additivity of the statistical dimension follows as in ref. [22], Eq. (3.45), for example (ii) See ref. [22], Lemma 3.5 and Eq. (3.34).

We now proceed to define conjugate representations.

Definition 4.39. Let $\rho$ be a locally irreducible representation of $\mathscr{C}_{0}$ localized in $I$. A locally irreducible representation $\bar{\rho}$ of $\mathscr{A}_{0}$ localized in $I$ is said to be conjugate to $\rho$ if $\rho \hat{\circ} \bar{\rho} \cong \bar{\rho} \hat{\circ} \rho$ contains the vacuum (identity) representation with multiplicity one. That is, there exists an isometry $R$ in $\bar{\rho} \hat{o} \rho\left(\mathscr{b}_{0}\right)^{\prime}$ such that

$$
\bar{\rho} \hat{\circ} \rho(A) R=R A \quad \forall A \in \mathscr{A}_{0} .
$$

It then follows that $R:=\varepsilon_{\rho \hat{o} \hat{\rho}}^{+} R$ satisfies

$$
\rho \hat{o} \bar{\rho}(A) \bar{R}=\bar{R} A \quad \forall A \in \mathscr{C}_{0} .
$$

Lemma 4.40. Let $\rho$ be localized in $I, \bar{\rho}$ a representation conjugate to $\rho$ and $\alpha \in S^{1}$ a point at infinity.

(i) If $\phi$ (resp. $\bar{\phi})$ is a left inverse for $\rho$ (resp. $\bar{\rho})$ on $\overline{\mathscr{b}}_{\alpha}^{d}$, then

$$
\phi\left(\varepsilon_{\rho \hat{o} \rho}^{+}\right)=\bar{\phi}\left(\varepsilon_{\bar{\rho} \hat{o} \bar{\rho}}^{+}\right),
$$

in particular, the statistical dimensions of $\rho$ and $\bar{\rho}$ coincide, $d(\rho)=d(\bar{\rho})$.

(ii) The representation $\bar{\rho}$ conjugate to $\rho$ is unique up to unitary equivalence.

Proof. Ad (i). The proof of Eq. (4.20) is identical to the one given in ref. [14] chapter III, as already noticed in ref. [22].

Ad (ii). The assertion follows just as in ref. [14], Theorem 3.3.

Guido and Longo [33] have given an explicit construction of the conjugate representation which we now adapt to our setting.

Definition 4.41. Let $h \in P S U(1 ; 1)$ and $K \subset S^{1}$ be an interval. Denote by $I_{K}$ the reflection about the endpoints of the interval $K$ (see Appendix I) and let

$$
j_{K}(A):=\operatorname{ad} J_{K}(A)=J_{K} A J_{K} \quad \forall A \in \mathscr{B}(\mathscr{H}),
$$

where $J_{K}$ is the modular conjugation of $\mathscr{A}(K)$. If $\rho$ is a representation of $\mathscr{C}_{0}$ localized in $I$, define

$$
\bar{\rho}_{J}^{K, h}(A):=\alpha_{h} \circ j_{K} \circ \rho_{I_{K} \cdot h^{-1} \cdot J} \circ j_{K} \circ \alpha_{h^{-1}}(A) \quad \forall A \in \mathscr{A}(J) .
$$

Theorem 4.42. (See ref. [33], Theorem 8.3). Let $\rho$ be a locally irreducible representation of $\mathscr{b}_{0}$ localized in $I$.

(i) The family $\bar{\rho}^{K, h}=\left\{\bar{\rho}_{J}^{K, h}\right\}_{J \subset S^{1}}$ defines a locally irreducible representation of Ao localized in $h \cdot I_{K} \cdot I$.

(ii) $\bar{\rho}^{K, h} \cong \bar{\rho}^{K^{\prime}, h^{\prime}}$, for all intervals $K, K^{\prime} \subset S^{1}$ and for all $h, h^{\prime} \in P S U(1 ; 1)$. 
(iii) The representation $\bar{\rho}^{K, h}$ transforms covariantly under the unitary representation of $\widetilde{P S U}(1 ; 1)$ given by

$$
\pi_{\bar{\rho}_{K, h}}^{M}(\tilde{g}):=\alpha_{K} \circ j_{K}\left(\pi_{\rho}^{M}\left(\operatorname{ad}_{I_{K} \cdot h^{-1}}(\tilde{g})\right)\right), \quad \tilde{g} \in \widetilde{P S U}(1 ; 1) .
$$

That is,

$$
\pi_{\bar{\rho}^{K, h}}^{M}(\tilde{g}) \bar{\rho}_{g^{-1}, J}^{K, h}(A) \pi_{\bar{\rho}^{K, h}}^{M}(\tilde{g})^{*}=\bar{\rho}_{J}^{K, h}\left(\alpha_{g}(A)\right) \quad \forall A \in \mathscr{A}\left(g^{-1} J\right),
$$

$\forall \tilde{g} \in \widetilde{P S U}(1 ; 1)$, where $g$ denotes as usual the image of $\tilde{g}$ by the canonical projection in $P S U(1 ; 1)$.

(iv) Let $J \supseteq I$ be an interval and $u$ a unitary operator implementing $\rho_{J}$ on $\mathscr{A}(J)$. If $h \in P S U(1 ; 1)$ is such that $h \cdot I_{J} \cdot I=I$ then the unitary operator $j_{J}\left(u^{*}\right)$ implements $\bar{\rho}_{J}^{J, h}$ on $\mathscr{A}(J)$.

For the convenience of the reader, a proof of this theorem is reproduced in appendix III.

Corollary 4.43. Let $\rho$ be a locally irreducible representation of $A_{0}$ localized in $I$ and having finite statistical dimension. If $J \supseteq I$ and $h \in P S U(1 ; 1)$ is such that $h \cdot I_{J} \cdot I=I$ then $\bar{\rho}^{J, h}$ is a representation conjugate to $\rho$.

Proof. By Theorem 4.43 (iv), we know that if $\rho_{J}$ is unitarily implemented by $u$ on $\mathscr{A}(J)$. Then $\bar{\rho}_{J}^{J, h}$ is unitarily implemented by $j_{J}\left(u^{*}\right)$ on $\mathscr{A}(J)$. Hence it follows from Lemma 2.1 of ref. [33] that $\bar{\rho}_{J}^{J, h}$ is a conjugate endomorphism of $\rho_{J}$ on $\mathscr{C}(J)$. Since $\rho$ has finite statistical dimension it follows from Theorem 4.34 (or directly from the generalized Pimsner-Popa inequality, see ref. [67], Th. 4.1) that the minimal index of the inclusion $\rho(A,(J)) \subseteq . A(J)$ is finite. Since $\rho$ is locally irreducible, we may apply Theorem 4.1 of ref. [68] and we obtain that

$$
\rho \circ \bar{\rho}(\mathscr{A}(J))^{\prime} \cap \mathscr{A}(J)
$$

contains an isometry such that

$$
\rho \circ \bar{\rho}(A) R=R A, \quad \forall A \in, \not b(J) .
$$

Furthermore, the endomorphism $\bar{\rho} \circ \rho$ of $\mathscr{A}(J)$ contains the identity representation with multiplicity one. By using explicitly Eq. (4.19), we see that

$$
\rho \hat{o} \bar{\rho}(A) R=R A \quad \forall A \in \mathscr{A}_{0},
$$

and that the representation $\rho \hat{o} \bar{\rho}$ of $\mathscr{A}_{0}$ contains the identity representation with multiplicity one. This completes the proof of the theorem.

Remark 4.44. Given a sector $[\rho]$ of $\mathscr{C}_{0}$ having finite statistical dimension, the map $[\rho] \rightarrow \overline{[\rho]}:=[\bar{\rho}]$ defines a conjugation on the set of sectors of . to having finite statistical dimension.

We conclude this section by summarizing well-known results on the fusion rule algebra associated to a list of representations of $\mathscr{t}_{0}$ and by presenting some natural conjectures concerning it.

Definition 4.45. Let $i, j$ and $k$ denote irreducible sectors of the conformal net $\mathscr{t}_{0}$ having finite statistical dimension. If $\rho_{i}$ (resp. $\rho_{j}$ ) is a representation of . $\ell_{0}$ belonging to the sector $i$ (resp. $j$ ), we denote by $N_{i j}^{k}$ the multiplicity of the sector $k$ in the 
decomposition of $\rho_{i} \hat{o} \rho_{j}$ (i.e., the number of irreducible representations belonging to the equivalence class $k$ in the direct sum decomposition of $\rho_{i} \hat{\imath} \rho_{j}$ ).

Let $L$ denote a list of irreducible sectors of the conformal net $\mathscr{B}_{0}$ having finite statistical dimension, closed under the decomposition of product representations and conjugation. The multiplicities $N_{i j}^{k}$ for $i, j, k \in L$ have the following well-known properties.

Lemma 4.46. (i) $N_{i j}^{k}=N_{\jmath i}^{k}=N_{\bar{k} i}^{\bar{j}}=N_{k \bar{i}}^{\bar{j}}$, for $i, j, k \in L$.

(ii) $N_{1 j}^{k}=\delta_{j \bar{j}}$, where $1 \in L$ denotes the sector of the vacuum (identity representation).

(iii) $\sum_{s \in L} N_{i j}^{s} N_{s k}^{l}=\sum_{i \in L} N_{i t}^{l} N_{j k}^{t}$, for $i, j, k, l \in L$.

(iv) $\sum_{l \in L} N_{p k}^{l} d(l)=d(p) d(k)$, where $d(l)$ denotes the statistical dimension of the sector $l \in L$.

Proof. See ref. [25] for example.

On the positive lattice $\mathbb{N}^{L}$, we define a distributive, associative and commutative product by setting

$$
i \times j=\sum_{k} N_{i j}^{k} k
$$

and an involutive and additive conjugation ${ }^{-}$by

$$
j \mapsto \bar{j}
$$

as in Remark 4.41. This defines a fusion rule algebra $\Phi$ in the sense of ref. [69]. The map

$$
i \mapsto \mathbb{N}_{i}=\left(N_{i j}^{k}\right)_{j, k \in L}
$$

defines a faithful representation of the fusion rule algebra $\Phi$ on the lattice $\mathbb{N}^{L}$ by nonnegative integer $|L| \times|L|$ matrices. These matrices are called fusion rule matrices. An extensive study of properties of fusion rule algebras derived from low-dimensional algebraic quantum field theories as well as a complete classification of those algebras generated by a single element having statistical dimension not exceeding two can be found in ref. [69]; (see in particular Th. 7.3.11).

We now restrict our attention to theories having only finitely many sectors, $|L|<\infty$, so called rational theories.

Definition 4.47. Given a sector $i \in L$, let $\rho_{i}$ be a representation belonging to the sector $i$ and $\pi_{\rho_{2}}^{M}$ be the unitary representation of $\widetilde{P S U}(1 ; 1)$ implementing the Moebius automorphisms of the net $\mathscr{A}_{0}$.

(i) The generator of rotations for the representation $\pi_{\rho_{i}}^{M}$ of $\widetilde{P S U}(1 ; 1)$ will be denoted by $K_{\rho_{i}}$ and the generator of rotations in the vacuum representation by $K$.

(ii) The (conformal) spin $s_{i}$ of the sector $i$ is defined by

$$
e^{2 \pi i s_{\imath}} \cdot \mathbb{1}_{\mathscr{B}}=\pi_{\rho_{\imath}}^{M}(R(2 \pi))=e^{2 \pi i K_{\rho_{i}}}
$$

where $R(2 \pi)$ denotes the rotation of $2 \pi$ in $\widetilde{P S U}(1 ; 1)$. The real number $s_{i}$ is defined modulo 1 and is an invariant for the sector $i$. Of course $s_{1}=0(\bmod 1)$ holds for the sector of the vacuum representation. 
As noticed in refs. [25] and [70], if a theory is rational one can define two matrices, $S$ and $T$, as follows:

$$
S:=\left(S_{i j}\right)_{i, \jmath \in L}, \quad \text { where } \quad S_{i j}:=\left(\sum_{\rho \in L} d(l)^{2}\right)^{-1 / 2} \psi_{i j},
$$

and

$$
\begin{aligned}
\psi_{i j} & :=\sum_{k \in L} N_{\imath \jmath}^{k} e^{2 \pi i\left(s_{\imath}+s_{\jmath}-s_{k}\right)} d(k), \\
T & :=\left(T_{i j}\right)_{i, j \in L}, \quad \text { where } \quad T_{i j}:=e^{2 \pi i\left(s_{j}-\sigma / 24\right)} \delta_{i j} .
\end{aligned}
$$

The constant $\sigma$, defined modulo 8 , is given by

$$
e^{-2 \pi i \sigma / 24}:=\left(\frac{\mu}{|\mu|}\right)^{1 / 3}, \quad \mu:=\sum_{i \in L} d(i)^{2} e^{-2 \pi i s_{\imath}} .
$$

If $S$ is invertible, it diagonalizes the fusion rule matrices $\mathbb{N}_{i}, i \in L$,

$$
N_{i k}^{m}=\sum_{j \in L} \frac{S_{k j} S_{i j} S_{j m}^{-1}}{S_{1 j}} .
$$

Furthermore, $S$ and $T$ are then unitary and satisfy the relations

$$
S^{2}=(T S)^{3}=C, \quad T C=C T=T,
$$

where $C=\left(\delta_{i j}\right)_{i, j \in L}$ is the charge conjugation matrix. That is, $S$ and $T$ generate a projective unitary representation of $\operatorname{PSL}(2 ; \mathbb{Z})$. As proven in ref. [70], $S$ is invertible, provided all sectors $i \in L$ satisfy braid statistics (i.e., provided $\varepsilon_{\rho_{i} \hat{\imath} \rho_{i}}^{+} \neq \varepsilon_{\rho_{\imath} \hat{\imath} \rho_{\imath}}^{-}$, for $\left.\rho_{i} \in i, \forall i \in L\right)$.

We are now ready to formulate some important conjectures, in analogy with ideas in rational conformal field theory.

Conjecture 4.48. Let $\Phi=\mathbb{N}^{L}$ be the fusion rule algebra of a rational conformal net $A_{0},|L|<\infty$. For $i \in L$ and $\rho_{\imath} \in i$, we expect the operator $e^{2 \pi i \tau K_{\rho_{\imath}}}$ to be trace class for $\operatorname{Im} \tau>0$.

Defining the specialized characters

$$
\chi_{\imath}(\tau):=\operatorname{tr} e^{2 \pi \imath \tau\left(K \rho_{i}-\sigma / 24\right)}, \quad i \in L
$$

where $\sigma$ is determined by Eq. (4.23), we conjecture that the modular transformations $\tau \rightarrow-\frac{1}{\tau}$ and $\tau \rightarrow \tau+1$ are implemented by unitary matrices $S^{\chi}=\left(S_{\imath j}^{\chi}\right)_{\imath, \jmath \in L}$ and $T^{\chi}=\left(T_{i j}^{\chi}\right)_{\imath, \jmath \in L}$

$$
\begin{aligned}
\chi_{\imath}\left(-\frac{1}{\tau}\right) & =\sum_{j \in L} S_{i j}^{\chi} \chi_{j}(\tau), \quad i \in L, \\
\chi_{i}(\tau+1) & =\sum_{j \in L} T_{i j}^{\chi} \chi_{j}(\tau), \quad i \in L,
\end{aligned}
$$

satisfying Eq. (4.24). As an immediate consequence of Eq. (4.25) and of the definition of $T$ one sees that $T=T^{\chi}$. Our main conjecture is that

$$
S^{\chi}=S
$$

also holds. 
It then follows from Eq. (4.26) that for $i \in L$,

$$
d(i)=\frac{S_{i 1}}{S_{11}}=\frac{S_{i 1}^{\chi}}{S_{11}^{\chi}}=\lim _{\tau \rightarrow i \infty} \frac{\sum_{k \in L} S_{i k}^{\chi} \chi_{k}(\tau)}{\sum_{l \in L} S_{1 l} \chi_{l}(\tau)}=\lim _{\tau \rightarrow 0} \frac{\chi_{\imath}(\tau)}{\chi_{1}(\tau)}
$$

This last equation characterizes $d(i)$ as the relative "size" of the sector $i \in L$ compared to the size of the vacuum sector [71]. (It is conceivable that a direct proof of (4.27) is easier than to proceed via a proof of (4.26).)

\section{IV.6. Superselection Structure of Conformal Nets Constructed from Vacuum Representations of Loop Groups}

We conclude this chapter by explaining how the conformal nets constructed in Sect. III.9 fit in the usual framework given by the algebraic theory of superselection sectors. We proceed by analogy with well-known results of algebraic and conformal quantum field theory.

Fix a positive level $m$ and consider the set $P_{+}^{(m)}$ of irreducible representations of $\widehat{L G}_{0}$. Let $\left(\pi_{m \hat{\Lambda}_{0}}, \mathscr{H}_{m \hat{\Lambda}_{0}}\right)$ be the vacuum representation of $\widehat{L G}_{0}$ at level $m$, $\mathscr{A}_{0}:=\left\{\mathscr{C}_{m} \hat{\Lambda}_{0}(I)\right\}_{I \subset S^{1}}$ the conformal net constructed in Sect. III.9 and $\pi_{0}^{M}$ the unitary representation of the Moebius group on $\mathscr{H}_{m \hat{\Lambda}_{0}}$ which implements the corresponding automorphisms of $\widehat{L G}_{0}$. In the context of loop groups, we obtain representations of the conformal net $t_{0}$ by the following lemma.

Lemma 4.49. Let $\hat{\Lambda} \in P_{+}^{(m)}$. The representations $\pi_{m \hat{\Lambda}_{0}}: \hat{\mathscr{b}}(I) \rightarrow \pi_{m \hat{\Lambda}_{0}}(\hat{\mathscr{b}}(I))$ and $\pi_{\hat{\Lambda}}: \hat{\mathscr{b}}(I) \rightarrow \pi_{\hat{\Lambda}}(\hat{\mathscr{b}}(I))$ of $\widehat{L G}_{0}$ determine a representation $\pi_{\hat{\Lambda}}=\left\{\pi_{\hat{\Lambda}, I}\right\}_{I \subset S^{1}}$ of the vacuum conformal net $\mathscr{b}_{0}=\left\{\mathscr{b}_{m \hat{\Lambda}_{0}}(I)\right\}_{I \subset S^{1}}$ such that for each $I \subset S^{1}$,

$$
\pi_{\hat{\Lambda}, I}: \mathscr{C}_{m \hat{\Lambda}_{0}}(I) \rightarrow \mathscr{A}_{\hat{\Lambda}}(I)
$$

is an isomorphism.

Proof. It follows from the proof of Theorem 3.3 that for each interval $I \subset S^{1}$ there exists a unitary operator $U: \mathscr{H}_{m} \hat{\Lambda}_{0} \rightarrow \mathscr{H}_{\hat{\Lambda}}$ such that

$$
U \pi_{m \hat{\Lambda}_{0}}(f)=\pi_{\hat{\Lambda}}(f) U, \quad \forall f \in \hat{\mathscr{b}}(I) .
$$

Hence the map

$$
\pi_{\hat{\Lambda}, I}\left(\pi_{m \hat{\Lambda}_{0}}(f)\right):=\pi_{\hat{\Lambda}}(f), \quad \forall f \in \hat{\mathscr{C}}(I)
$$

extends to an isomorphism $\pi_{\hat{\Lambda}, I}=\mathscr{b}_{m \hat{\Lambda}_{0}}(I) \rightarrow \mathscr{b}_{\hat{\Lambda}}(I)$ for all intervals $I \subset S^{1}$. The consistence of these representations, Moebius covariance and the spectrum condition are immediate consequences of Eq. (4.28). This verifies all properties of Definition 4.1 and completes the proof of the lemma.

If $\hat{\Lambda} \in P_{+}^{(m)}$, we will also denote by $\hat{\Lambda}$ the corresponding superselection sector obtained by the preceding lemma. It follows from Lemma 4.49 and Lemma 4.6 that every representation $\left\{\pi_{\hat{\Lambda}, I}\right\}_{I \subset S^{1}}$ of $\mathscr{C}_{0}$ can be obtained by composing the vacuum representation $\pi_{m \hat{\Lambda}_{0}}$ with a localized endomorphism $\rho$ of $\mathscr{b}_{0}$. We may therefore 
apply the general theory presented in Sects. IV.1-IV.5 to define a composition of representations, unitary braid group representations, etc.

What is missing to complete the picture is a mathematically rigorous result showing that the statistical dimension, $d(\hat{\Lambda})$, of every sector $\hat{\Lambda}$ is finite and permitting to compute $d(\hat{\Lambda})$ explicitly. However, assuming that the conjecture described in Eq. (4.27) of the previous section holds, this gap can be filled. From Eq. (4.27) and formulas in [62] we obtain the following expression for $d(\hat{\Lambda})$ :

$$
d(\hat{\Lambda})=\lim _{\tau \rightarrow 0} \frac{\chi_{\hat{\Lambda}}(\tau ; \mathscr{G})}{\chi_{m \hat{\Lambda}_{0}}(\tau ; \mathscr{G})}
$$

where $\chi_{\hat{\Lambda}}(\tau ; \mathscr{G})$ are the specialized characters of Kac and Wakimoto (see ref. [62], Sect. 4.8). It follows from the asymptotic expansion given in Sect. III.8 and Eq. (2.2.1) of ref. [62] that

$$
d(\hat{\Lambda})=\frac{a(\hat{\Lambda})}{a\left(m \hat{\Lambda}_{0}\right)}=\operatorname{tr}_{\Lambda} \exp \left(\frac{2 \pi i \nu^{-1}(\rho)}{m+g}\right),
$$

where the trace is taken in the irreducible, finite-dimensional $\mathscr{G}$-module with highest weight $\Lambda$, and $\nu^{-1}(\rho)$ is the inverse image of $\rho=\sum_{\imath=1}^{l} \Lambda_{\imath}$ in the Cartan subalgebra $\mathfrak{h}$ of $\mathscr{G}$ by the isomorphism $\nu$ introduced in Sect. III.1. Due to Theorem 4.35, this formula coincides with a conjecture of Wassermann formulated in ref. [29]

Explicit formulas for the $S$ and $T$ matrices are also well-known in this context; (see ref. [62], Eqs. (2.1.5)-(2.1.11)):

$$
T=\left(T_{\hat{\Lambda}_{1} \hat{\Lambda}_{2}}\right)_{\hat{\Lambda}_{1}, \hat{\Lambda}_{2}} \in P_{+}^{(m)}, \quad T_{\hat{\Lambda}_{1} \hat{\Lambda}_{2}}=e^{2 \pi \imath s} \hat{\Lambda}_{1} \delta_{\hat{\Lambda}_{1} \hat{\Lambda}_{2}}
$$

where

$$
s_{\hat{\Lambda}}:=\frac{(\hat{\Lambda}+\hat{\rho} \mid \hat{\Lambda}+\hat{\rho})}{2(m+g)}-\frac{(\hat{\rho} \mid \hat{\rho})}{2 g}
$$

is called the modular anomaly by Kac and Wakimoto. Applying the so-called "strange" formula (see ref. [62], Eq. (1.1.1)), we obtain that

$$
s_{\hat{\Lambda}}=h_{\hat{\Lambda}}-\sigma,
$$

(see Sect. III.7 for the definition of $h_{\hat{\Lambda}}$ and $\sigma$ ) so that $h_{\hat{\Lambda}}$ coincides with the spin of Definition 4.47 and $\sigma$ with the constant defined in Eq. (4.23).

For the $S$ matrix one has

$$
\begin{aligned}
S & =\left(S_{\hat{\Lambda}_{1} \hat{\Lambda}_{2}}\right)_{\hat{\Lambda}_{1}, \hat{\Lambda}_{2}} \in P_{+}^{(m)}, \\
S_{\hat{\Lambda}_{1} \hat{\Lambda}_{2}} & =a\left(\hat{\Lambda}_{1}\right) \operatorname{tr}_{\Lambda_{2}}\left(\exp \frac{-2 \pi i \nu^{-1}\left(\Lambda_{1}+\rho\right)}{m+g}\right), \\
a\left(\hat{\Lambda}_{1}\right) & =\operatorname{tr}_{\Lambda_{1}}\left(\exp \frac{-2 \pi i \nu^{-1}(\rho)}{m+g}\right) \cdot\left(\sum_{\hat{\Lambda} \in P_{+}^{(m)}}\left(\operatorname{tr}_{\Lambda} \exp \frac{-2 \pi i \nu^{-1}(\rho)}{m+g}\right)^{2}\right)^{-1 / 2} .
\end{aligned}
$$

Given $\hat{\Lambda} \in P_{+}^{(m)}$, let ${ }^{t} \hat{\Lambda}:=m \hat{\Lambda}_{0}+{ }^{t} \Lambda$, where for a $\mathscr{G}$-module with highest weight $\Lambda$, ${ }^{t} \Lambda$ is defined as the highest weight of the contragredient $\mathscr{G}$-module. It follows from

$$
C=S^{2}=\left(\delta_{\hat{\Lambda}^{t} \hat{\Lambda}}\right) \text {, }
$$


where the first equality is given by Eqs. (4.24), (4.26) and the second follows by Eq. (2.1.11) of ref. [62] that ${ }^{t} \hat{\Lambda}$ is the conjugate sector to $\hat{\Lambda}$.

Finally, we remark that the braid group representations of Sect. IV.3 are expected to be of Hecke [72] or of Birman-Wenzl [73] type in these examples.

In conclusion, we have proven partial results strongly suggesting that the irreducible representations of a loop group at fixed level provide examples of algebraic conformal field theories with finitely many superselection sectors corresponding to chiral WZW-models [74].

For a complementary approach to these problems and complete results in the examples of the chiral Ising model and a class of level-one Kac-Moody algebras see [80].

\section{Appendix I. Notation and Geometry}

Let $S^{1}:=\{z \in \mathbb{C}|| z \mid=1\}$ be the unit circle in $\mathbb{C}$ and $S_{ \pm}:=\left\{z \in S^{1} \mid \operatorname{Im} z \gtrless 0\right\}$ its upper and lower part. If we denote by $I$ any open, non-dense interval of the circle then,

$$
I^{\prime}:=\left(S^{1} \backslash I\right)^{0}
$$

is the interior of its complement in $S^{1}$ : The group $S U(1 ; 1)$,

$$
S U(1 ; 1):=\left\{A=\left.\left(\begin{array}{cc}
\alpha & \beta \\
\bar{\beta} & \bar{\alpha}
\end{array}\right) \in M_{2}(\mathbb{C})|| \alpha\right|^{2}-|\beta|^{2}=1\right\}
$$

acts as a transformation group on $S^{1}$ :

$$
\begin{aligned}
(A ; z) \in S U(1 ; 1) \times S^{1} \mapsto A \cdot z & =\left(\begin{array}{cc}
\alpha & \beta \\
\bar{\beta} & \bar{\alpha}
\end{array}\right) \cdot z \\
: & =\frac{\alpha z+\beta}{\bar{\beta} z+\bar{\alpha}} .
\end{aligned}
$$

This action factors to a transitive action of $P S U(1 ; 1)=S U(1 ; 1) /\{ \pm \mathbb{1}\} . P S U(1 ; 1)$ is the group of fractional linear transformations of the circle (Moebius group).

The geometric interpretation of the action of $S U(1 ; 1)$ on $S^{1}$ is most easily seen by performing the stereographic projection of $S^{1} \backslash\{-1\}$ onto $\mathbb{R}$ which maps $-1 \in S^{1}$ on $\infty$ :

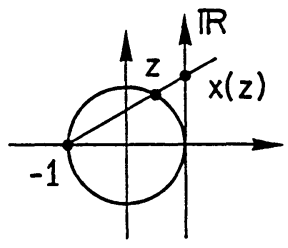

$$
z \in S^{1} \backslash\{-1\} \mapsto x(z) \in \mathbb{R}
$$

it is given by

$$
x:=(-i) \frac{z-1}{z+1}, \quad x( \pm i)= \pm 1
$$


Setting $T:=\left(\begin{array}{rr}-i & i \\ 1 & 1\end{array}\right)$ we may write

$$
x=T \cdot z
$$

using the same notation as in Eq. (AI.1). Conjugation of $S U(1 ; 1)$ by $T$ provides an isomorphism of $S U(1 ; 1)$ onto $S L(2 ; \mathbb{R})$,

$$
\begin{aligned}
\psi: S U(1 ; 1) & \stackrel{\cong}{\longmapsto} S L(2 ; \mathbb{R}) \\
A & \longmapsto \tilde{A}:=\psi(A), \\
& :=T A T^{-1} .
\end{aligned}
$$

In general, we will denote elements of $S U(1 ; 1)$ by capital latin letters and the corresponding elements in $S L(2 ; \mathbb{R})$ by the same letter tilded. If $A=\left(\begin{array}{cc}\alpha & \beta \\ \bar{\beta} & \bar{\alpha}\end{array}\right) \in$ $S U(1 ; 1)$ then $\tilde{A}=\left(\begin{array}{ll}\operatorname{Re} \alpha-\operatorname{Re} \beta & \operatorname{Im} \alpha+\operatorname{Im} \beta \\ \operatorname{Im} \beta-\operatorname{Im} \alpha & \operatorname{Re} \alpha+\operatorname{Re} \beta\end{array}\right):=\left(\begin{array}{cc}a & b \\ c & d\end{array}\right) \in S L(2 ; \mathbb{R})$. It follows from Eqs. (AI.1)-(AI.3) that the action of $S U(1 ; 1)$ on $S^{1}$ corresponds to the action of $S L(2 ; \mathbb{R})$ on $\mathbb{R}$ as fractional linear transformations,

$$
(\tilde{A} ; x) \in S L(2 ; \mathbb{R}) \times \mathbb{R} \cdot x \longmapsto \tilde{A} \cdot x:=\frac{a x+b}{c x+d} \in \mathbb{R} \cup\{\infty\} .
$$

Only the subgroup of $S U(1 ; 1)$ which leaves - 1 fixed corresponds to well-defined transformations of $\mathbb{R}$ onto itself. This subgroup is mapped by $\psi$ onto the subgroup of matrices of the form $\left(\begin{array}{ll}a & b \\ 0 & a^{-1}\end{array}\right)$ of $S L(2 ; \mathbb{R})$. We call this subgroup the Poincaré subgroup, since it corresponds to translations and dilatations (see below).

We now define the one-parameter subgroups of $S L(2 ; \mathbb{R})$ which are used in Chap. II of this work. We also exhibit the corresponding subgroups of $S U(1 ; 1)$ (obtained by applying the isomorphism $\psi^{-1}$ ).

(i) Translations

$$
\begin{aligned}
p \in \mathbb{R} \longmapsto \tilde{T}(p) & =\left(\begin{array}{cc}
1 & p \\
0 & 1
\end{array}\right) \in S L(2 ; \mathbb{R}) \longleftrightarrow \\
T(p) & =\left(\begin{array}{cc}
1+i p / 2 & i p / 2 \\
-i p / 2 & 1-i p / 2
\end{array}\right) \in S U(1 ; 1) \\
\tilde{T}(p) \cdot x & =x+p, \quad x \in \mathbb{R}
\end{aligned}
$$

(ii) Dilatations.

$$
\begin{aligned}
t \in \mathbb{R} \longmapsto \tilde{D}(t) & =\left(\begin{array}{cc}
e^{-\pi t} & 0 \\
0 & e^{\pi t}
\end{array}\right) \in S L(2 ; \mathbb{R}) \longleftrightarrow \\
D(t) & =\left(\begin{array}{cc}
\operatorname{ch} \pi t & \operatorname{sh} \pi t \\
\operatorname{sh} \pi t & \operatorname{ch} \pi t
\end{array}\right) \in S U(1 ; 1) . \\
\tilde{D}(t) \cdot x & =e^{-2 \pi t} \cdot x, \quad x \in \mathbb{R} .
\end{aligned}
$$


(iii) Special conformal transformations

$$
\begin{aligned}
n \in \mathbb{R} \longmapsto \tilde{S}(n) & :=\left(\begin{array}{cc}
1 & 0 \\
n & 1
\end{array}\right) \in S L(2 ; \mathbb{R}) \longleftrightarrow \\
S(n) & =\left(\begin{array}{rr}
1-i n / 2 & i n / 2 \\
-i n / 2 & 1+i n / 2
\end{array}\right) \in S U(1 ; 1) . \\
\tilde{S}(n) \cdot x & =\frac{x}{n x+1} \in \mathbb{R} \cup\{\infty\}, \quad x \in \mathbb{R} .
\end{aligned}
$$

(iv) Rotations on $\mathbb{R}$.

$$
\begin{aligned}
t \in \mathbb{R} / 2 \mathbb{Z} \longmapsto \tilde{R}(t) & =\left(\begin{array}{rr}
\cos \pi t & \sin \pi t \\
-\sin \pi t & \cos \pi t
\end{array}\right) \in S L(2 ; \mathbb{R}) \longleftrightarrow \\
R(t) & =\left(\begin{array}{cc}
e^{i \pi t} & 0 \\
0 & e^{-i \pi t}
\end{array}\right) \in S U(1 ; 1) . \\
\tilde{R}(t) \cdot x & =\frac{\cos \pi t x+\sin \pi t}{-\sin \pi t \cdot x+\cos \pi t} \in \mathbb{R} \cup\{\infty\}, \quad x \in \mathbb{R} .
\end{aligned}
$$

The following elementary facts about the structure of $S L(2 ; \mathbb{R})$ will be useful. $S L(2 ; \mathbb{R})$ is a simple, real, non-compact Lie group. Each element $A$ of $S L(2 ; \mathbb{R})$ can be uniquely decomposed as a product of translations, dilatations and rotations:

$$
\begin{aligned}
A & =\left(\begin{array}{ll}
a & b \\
c & d
\end{array}\right) \\
& =\left(\begin{array}{cc}
1 & \frac{a c+b d}{c^{2}+d^{2}} \\
0 & 1
\end{array}\right) \cdot\left(\begin{array}{cc}
\left(c^{2}+d^{2}\right)^{-1 / 2} & 0 \\
0 & \left(c^{2}+d^{2}\right)^{1 / 2}
\end{array}\right) \cdot \frac{1}{\sqrt{c^{2}+d^{2}}}\left(\begin{array}{cc}
d & -c \\
c & d
\end{array}\right)
\end{aligned}
$$

(Iwasawa decomposition). That is, as a manifold,

$$
\begin{aligned}
S L(2 ; \mathbb{R}) & \cong \underline{T} \times \underline{D} \times \underline{R}, \text { where } \\
\underline{T} & :=\left\{\tilde{T}(p)=\left(\begin{array}{cc}
1 & p \\
0 & 1
\end{array}\right) \mid p \in \mathbb{R}\right\}, \\
\underline{D} & :=\left\{\tilde{D}(t)=\left(\begin{array}{cc}
e^{-\pi t} & 0 \\
0 & e^{\pi t}
\end{array}\right) \mid t \in \mathbb{R}\right\}, \\
\underline{R} & :=\left\{\tilde{R}(t)=\left(\begin{array}{cc}
\cos \pi t & \sin \pi t \\
-\sin \pi t & \cos \pi t
\end{array}\right) \mid t \in \mathbb{R} / 2 \mathbb{Z}\right\},
\end{aligned}
$$

are the one-parameter subgroups of translations, dilatations and rotations, respectively. It follows that $S L(2 ; \mathbb{R})$ is connected and that its fundamental group is infinite cyclic.

The Iwasawa decomposition of a special conformal transformation is

$$
\left(\begin{array}{cc}
1 & 0 \\
\operatorname{tg} \theta & 1
\end{array}\right)=\left(\begin{array}{cc}
1 & \sin \theta \cos \theta \\
0 & 1
\end{array}\right)\left(\begin{array}{cc}
\cos \theta & 0 \\
0 & \frac{1}{\cos \theta}
\end{array}\right)\left(\begin{array}{cc}
\cos \theta & -\sin \theta \\
\sin \theta & \cos \theta
\end{array}\right)
$$

for $\theta \in\left(\frac{-\pi}{2}, \frac{\pi}{2}\right)$. This implies that the one-parameter subgroups of translations, dilatations and special conformal transformations generate $S L(2 ; \mathbb{R})$. 
The following convention will be used to describe intervals on $S^{1}$. If $z, w \in S^{1}$ then $(z ; w)$ is defined as the interval on $S^{1}$ which is covered by walking from $z$ to $w$ in anticlockwise direction (direction of increasing angle coordinate):

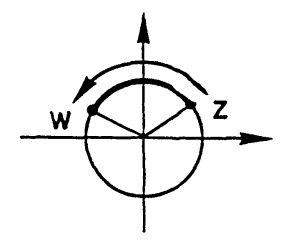

The interval $(w ; z)$ is then the complement of $(z ; w):(w ; z)=(z ; w)^{\prime}$. For example, $S_{+}=(1 ;-1)$ and $S_{-}=(-1 ; 1)=S_{+}^{\prime}$.

We now introduce two geometric transformations on $\mathbb{R}$ and on $S^{1}$ which will be of importance.

(i) The reflection about $(0 ; \infty)$ on $\mathbb{R} . x \mapsto-x$.

If we define $\tilde{I}_{(0 ; \infty)}:=\left(\begin{array}{rr}-1 & 0 \\ 0 & 1\end{array}\right)$, then $\tilde{I}_{(0 ; \infty)} \cdot x=-x$ holds, where $\tilde{I}_{(0 ; \infty)}$ acts as a fractional linear transformation on $\mathbb{R}$. The image of $\tilde{I}_{(0 ; \infty)}$, by $\psi^{-1}$ is $\left(\begin{array}{ll}0 & 1 \\ 1 & 0\end{array}\right)$. This induces a map of $S^{1}$ onto itself, $z \mapsto\left(\begin{array}{ll}0 & 1 \\ 1 & 0\end{array}\right) \cdot z=\frac{1}{z}$, which is a reflection about the axis passing through -1 and 1 . We define

$$
I_{S_{+}}=I_{(1 ;-1)}:=\left(\begin{array}{cc}
0 & 1 \\
1 & 0
\end{array}\right) \text {. }
$$

(ii) The reflection about $(-1 ; 1)$ on $\mathbb{R} . x \mapsto \frac{1}{x} \in \mathbb{R} \cup\{\infty\}$.

We set $\tilde{I}_{(-1 ; 1)}:=\left(\begin{array}{rr}0 & -1 \\ -1 & 0\end{array}\right)$, so that $\tilde{I}_{(-1 ; 1)} \cdot x=\frac{1}{x}$. The corresponding fractional linear transformation on $S^{1}$ is given by the matrix $I_{(-\imath ; i)}:=\psi^{-1}\left(I_{(-1 ; 1)}\right)=$ $\left(\begin{array}{rr}0 & -i \\ i & 0\end{array}\right)$. The map $z \mapsto I_{(-i ;))} \cdot z=-\frac{1}{z}$ is a reflection about the axis going through $\pm i$.

We define the group of improper Moebius transformations, $P S U(1 ; 1)_{ \pm}$as the group of transformations of $S^{1}$ generated by the Moebius group and the inversion $I_{S_{+}}$. This group is obtained from

$$
S U(1 ; 1)_{ \pm}:=\left\{A=\left.\left(\begin{array}{cc}
\alpha & \beta \\
\bar{\beta} & \bar{\alpha}
\end{array}\right) \in M_{2}(\mathbb{C})|| \alpha\right|^{2}-|\beta|^{2}= \pm 1\right\}
$$

by factoring out the subgroup which corresponds to the identity transformation, that is, $\{ \pm \mathbb{1}\}$. Hence,

$$
P S U(1 ; 1)_{ \pm}=S U(1 ; 1)_{ \pm} /\{ \pm \mathbb{1}\} .
$$


The image of $P S U(1 ; 1)_{ \pm}$by $\psi=\operatorname{ad} T$ is

$$
\begin{aligned}
P S L(2 ; \mathbb{R})_{ \pm} & =S L(2 ; \mathbb{R})_{ \pm} /\{ \pm \mathbb{1}\} \\
S L(2 ; \mathbb{R})_{ \pm} & :=\left\{\tilde{A}=\left(\begin{array}{ll}
a & b \\
c & d
\end{array}\right) \in M_{2}(\mathbb{R}) \mid \operatorname{det} \tilde{A}= \pm 1\right\}
\end{aligned}
$$

$S U(1 ; 1)$ is a normal subgroup of $S U(1 ; 1)_{ \pm}$of order two. Let $\phi$ be the outer automorphism of $S U(1 ; 1)$ defined by

$$
\phi: A=\left(\begin{array}{cc}
\alpha & \beta \\
\bar{\beta} & \bar{\alpha}
\end{array}\right) \mapsto I_{S_{+}} \cdot A \cdot I_{S_{+}}=\left(\begin{array}{cc}
\bar{\alpha} & \bar{\beta} \\
\beta & \alpha
\end{array}\right)=\bar{A}, \quad \phi^{2}=\mathrm{id}
$$

where the bar over $A$ means complex conjugation. It then follows that $S U(1 ; 1)_{ \pm}$is the semi-direct product of $S U(1 ; 1)$ and $\left\{\mathbb{1} ; I_{S_{+}}\right\}$with respect to $\phi$.

We now proceed to define a reflection about two arbitrary points $z, w \in S^{1}$. Let $N_{(z ; w)} \in S U(1 ; 1)$ be a transformation which maps +1 in $z,-1$ in $w$ and the interval $(1 ;-1)=S_{+}$onto the interval $(z ; w)$. Then we set

$$
I_{(z ; w)}:=N_{(z ; w)} I_{(1 ;-1)} N_{(z ; w)}^{-1} \in S U(1 ; 1)_{ \pm}
$$

If $N_{(z ; w)}^{(1)}$ and $N_{(z ; w)}^{(2)}$ are two transformations which map 1 in $z,-1$ in $w$ and $S_{+}$onto $(z ; w)$, then

$$
B=N_{(z ; w)}^{(1)-1} N_{(z ; w)}^{(2)} \in S U(1 ; 1)
$$

maps $S_{+}$onto $S_{+}$and leaves \pm 1 fixed. Hence $B= \pm D(t)$ for some $t \in \mathbb{R}$ and, since $B I_{S_{+}} B^{-1}=I_{S_{+}}$, it follows that $I_{(z ; w)}$ is independent of the particular choice of $N_{(z ; w)}$.

Since $I_{(-1 ; 1)}=R(1 / 2) I_{(1 ;-1)} R(-1 / 2)=-I_{(1 ;-1)}$ it follows that $I_{(z ; w)}=$ $-I_{(w ; z)} \forall z, w \in S^{1}$, so that $I_{(z ; w)}$ and $I_{(w ; z)}$ induce the same fractional linear transformation in $P S U(1 ; 1)_{ \pm}$. This transformation is the reflection about $z$ and $w$.

In the real picture it is easy to compute $\tilde{I}_{(x ; y)}:=\psi\left(I_{(z ; w)}\right)$, where $x$ and $y$ are the images of $z$ and $w$ by stereographic projection. If $x, y \in \mathbb{R}, x<y$, then

$$
\tilde{I}_{(x ; y)}=\frac{1}{y-z}\left(\begin{array}{cc}
-(x+y) & 2 x y \\
-2 & x+y
\end{array}\right)
$$

whereas,

$$
\tilde{I}_{(x ; \infty)}=\left(\begin{array}{rl}
-1 & 2 x \\
0 & 1
\end{array}\right), \quad x \in \mathbb{R}
$$

The group $S U(1 ; 1)_{ \pm}$is generated by reflections. This fact is most easily checked in the real picture using Eqs. (AI.4), (AI.5). It is sufficient to write translations, dilatations and special conformal transformations as products of reflections:

$$
\begin{aligned}
& \tilde{T}(p)=\tilde{I}_{(p / 2 ; \infty)} \tilde{I}_{(0 ; \infty)}, \\
& \tilde{S}(n)=\tilde{I}_{(0 ; 2 / n)} \tilde{I}_{(0 ; \infty)}, \\
& \tilde{D}(t)=\tilde{I}_{\left(-e^{-\pi t} ; e^{-\pi t}\right)} \tilde{I}_{(-1 ; 1)} .
\end{aligned}
$$




\section{Appendix II. Proof of Lemma 2.22}

Proof of Lemma 2.22. Let $U \in \mathscr{G}$. Since $S U(1 ; 1)_{ \pm}$is generated by reflections and

$$
\begin{aligned}
J_{(\alpha ; \beta)} & =\hat{\pi}_{0}\left(I_{(\alpha ; \beta)}\right), \\
\Delta_{(\alpha ; \beta)}^{i t} & =\pi_{0}\left(N_{(\alpha ; \beta)}\right) \pi_{0}(D(t)) \pi_{0}\left(N_{(\alpha ; \beta)}\right)^{*}
\end{aligned}
$$

for $N_{(\alpha ; \beta)} \in S U(1 ; 1)$ such that $N_{(\alpha ; \beta)} \cdot S_{+}=(\alpha ; \beta)$, it follows that $U \in \tilde{\mathscr{G}}$ is equivalent to

$$
U J_{I}=J_{I} U, \quad U \Delta_{I}^{\imath t}=\Delta_{I}^{i t} U, \quad \forall I \subset S^{1} .
$$

By the elementary result of Takesaki already used in the proof of Theorem 2.19, every $U \in \mathscr{G}$ commutes with $\Delta_{I}^{i t}, J_{I}, \forall I \subset S^{1}$. Hence $U$ satisfies (AII.l).

This completes the proof of the lemma.

Proof of Lemma 2.24. We only need to prove that if $U \in \tilde{\mathscr{G}}$ and $\Omega$ is cyclic for $\mathscr{B}$ then $U \in \mathscr{G}$.

Since $\Omega$ is cyclic for $\mathscr{B}$, it follows from the Reeh-Schlieder theorem (Corollary 2.8 ) that $\Omega$ is cyclic and separating for $\mathscr{A}^{U}(J), \forall J \subset S^{1}$. For each $I \subset S^{1}$ we define a von Neumann algebra $\tilde{\mathscr{b}}(I)$ by

$$
\tilde{f}(I):=U \mathscr{C}(I) U^{*} .
$$

Since $U$ leaves the vacuum vector invariant $\Omega$ is cyclic and separating for $\tilde{\mathscr{A}}(I)$, so that $\tilde{J}_{I}, \tilde{\Delta}_{I}^{i t}$, the modular conjugation and the modular group associated with $\tilde{\mathscr{b}}(I)$ and $\Omega$, are well defined. Clearly,

$$
\tilde{\Delta}_{I}^{i t}=U A_{I}^{\imath t} U^{*}, \quad \tilde{J}_{I}=U J_{I} U^{*} .
$$

But by assumption $U$ commutes with $J_{I}$ and $\Delta_{I}^{i t}$, that is, $\tilde{J}_{I}=J_{I}, \tilde{\Delta}_{I}^{i t}=\Delta_{I}^{i t}$.

Since $\Omega$ is cyclic and separating for

$$
\mathscr{C}^{U}\left(I^{\prime}\right)=\mathscr{A}\left(I^{\prime}\right) \cap \tilde{\mathscr{b}}\left(I^{\prime}\right),
$$

it follows that $\Omega$ is cyclic and separating for

$$
\mathscr{C}:=\mathscr{A}(I) \vee \tilde{\mathscr{b}}(I)
$$

by taking commutants. Since $S_{I}=J_{I} \Delta_{I}^{1 / 2}=\tilde{J}_{I} \tilde{\Delta}_{I}^{1 / 2}=\tilde{S}_{I}$ and $\Omega$ is separating for $\mathscr{C}$, it follows that $\mathscr{b}(I)=\tilde{\mathscr{b}}(I)$. This is a variant of Lemma 3 of ref. [75], Sect. 10.5. This completes the proof of the lemma.

Proof of Lemma 2.27. It is sufficient to find a conjugation $J_{\pi}$ satisfying Eq. (2.22). Clearly, if $J_{\pi}^{1}$ and $J_{\pi}^{2}$ satisfy Eq. (2.22) then $J_{\pi}^{1} J_{\pi}^{2}$ is a unitary operator commuting with the irreducible representation $\pi$ of $S U(1 ; 1)$ and consequently a phase factor. Hence $J_{\pi}$, if it exists, is unique up to an uninteresting phase factor.

We now construct $J_{\pi}$ explicitly in the real picture. When $S U(1 ; 1)$ is mapped onto $S L(2 ; \mathbb{R})$, the outer automorphism $A \rightarrow \bar{A}, A \in S U(1 ; 1)$ corresponds to conjugation by $\tilde{I}_{(0 ; \infty)}=\left(\begin{array}{rr}-1 & 0 \\ 0 & 1\end{array}\right)$ (see Appendix I). The one-parameter subgroups

$$
\begin{gathered}
\hat{R}(\theta):=\left(\begin{array}{rr}
\cos \theta & \sin \theta \\
-\sin \theta & \cos \theta
\end{array}\right), \quad \hat{D}(t):=\left(\begin{array}{cc}
e^{-t / 2} & 0 \\
0 & e^{t / 2}
\end{array}\right), \\
\hat{A}(s):=\left(\begin{array}{ll}
\operatorname{ch} s / 2 & \operatorname{sh} s / 2 \\
\operatorname{sh} s / 2 & \operatorname{ch} s / 2
\end{array}\right)
\end{gathered}
$$


have infinitesimal generators

$$
\mathfrak{z}:=\left.\frac{d}{d \theta}\right|_{\theta=0} \hat{R}(\theta), \quad a:=\left.\frac{d}{d t}\right|_{t=0} \hat{D}(t), \quad b:=\left.\frac{d}{d s}\right|_{s=0} \hat{H}(s)
$$

with the Lie algebra relations

$$
[\mathfrak{z} ; a]=2 b, \quad[\mathfrak{z} ; b]=-2 a, \quad[a ; b]=-\frac{1}{2} \mathfrak{z} .
$$

Let $x_{+}:=a-i b, x_{-}:=a+i b$.

An irreducible representation $\pi$ of $S L(2 ; \mathbb{R})$ of the holomorphic discrete series decomposes into a direct sum of one-dimensional eigenspaces of $\pi(\mathfrak{z})$ with orthonormal basis vectors $\left\{v_{k}\right\}_{k=n, n+2, \ldots} n \in \mathbb{Z}_{+}$satisfying

$$
\begin{aligned}
\pi(\mathfrak{z}) v_{k} & =i k v_{k}, \\
\pi\left(x_{+}\right) v_{k} & =\alpha_{k} v_{k+2}, \\
\pi\left(x_{-}\right) v_{k+2} & =-\alpha_{k} v_{k},
\end{aligned}
$$

where $\alpha_{k}:=\frac{1}{2}\left[(k+1)^{2}-(n-1)^{2}\right]^{1 / 2}$. The commutation relations $(2.22)$ are equivalent to the infinitesimal relations

$$
\pi(\mathfrak{z}) J_{\pi}=-J_{\pi} \pi(\mathfrak{z}), \quad \pi\left(x_{ \pm}\right) J_{\pi}=J_{\pi} \pi\left(x_{ \pm}\right)
$$

so that if we define $J_{\pi}$ as the unique antilinear conjugation which maps $v_{k}$ to $v_{k}$, one checks using Eqs. (AII.2) that Eqs. (AII.3) are satisfied. This completes the proof of the lemma.

Lemma AII.1. Let $\hat{\pi}$ be a corepresentation of $S U(1 ; 1)_{ \pm}$on $\mathscr{H}$, assume that its restriction $\pi$ to $S U(1 ; 1)$ has positive generator of rotations. Then there exists a Hilbert space $\mathscr{H}^{\prime}$, a corepresentation $\hat{\pi}^{\prime}$ of $S U(1 ; 1)_{ \pm}$on $\mathscr{H}^{\prime}$ and an invertible isometry $V: \mathscr{H} \rightarrow \mathscr{H}^{\prime}$ intertwining $\hat{\pi}$ and $\hat{\pi}^{\prime}$ such that

(i) $\mathscr{H}^{\prime}=\mathscr{H}_{1} \otimes \mathscr{H}_{\pi^{1}} \oplus \mathscr{H}_{2} \otimes \mathscr{H}_{\pi^{2}} \oplus \ldots \oplus \mathscr{H}_{\infty} \otimes \mathscr{H}_{\pi^{\infty}}, \operatorname{dim} \mathscr{H}_{2}=i$,

(ii) $\pi^{\prime}=\mathbb{1}_{1} \otimes \pi^{1} \oplus \mathbb{1}_{2} \otimes \pi^{2} \oplus \ldots \oplus \mathbb{1}_{\infty} \otimes \pi^{\infty}$,

where the $\pi^{i}$ 's are disjoint, multiplicity-free direct sums of holomorphic discrete series representations of $S U(1 ; 1){ }^{8}$

$$
\hat{\pi}^{\prime}\left(I_{S_{+}}\right)=\hat{J}_{\pi^{1}}\left(e^{1}\right) \oplus \hat{J}_{\pi^{2}}\left(e^{2}\right) \oplus \ldots \oplus \hat{J}_{\pi^{\infty}}\left(e^{\infty}\right),
$$

the notation being just as in Theorem 2.26.

Proof. Every direct sum of discrete series representations of $S U(1 ; 1)$ can be brought into the form given in (i), (ii), [76], so that we may assume that $\mathscr{H}=\mathscr{H}^{\prime}$ and $\pi=\pi^{\prime}$. It remains only to prove that there exists a unitary operator $U$ commuting with $\pi$ and such that

$$
U \hat{\pi}\left(I_{S_{+}}\right) U^{*}=\hat{\pi}^{\prime}\left(I_{S_{+}}\right) .
$$

Choose orthonormal bases $e^{i}$ in $\mathscr{H}_{i}$ and define $\hat{\pi}^{\prime}\left(I_{S_{+}}\right)$by means of Eq. (AII.4). It then follows that

$$
\pi^{\prime}\left(I_{S_{+}}\right) \pi(A) \pi^{\prime}\left(I_{S_{+}}\right)=\pi(\bar{A}) \quad \forall A \in S U(1 ; 1) .
$$

8 possibly trivial 
Hence $\hat{\pi}\left(I_{S_{+}}\right) \hat{\pi}^{\prime}\left(I_{S_{+}}\right)$commutes with $\pi(S U(1 ; 1))$ and by Schur's Lemma,

$$
\hat{\pi}\left(I_{S_{+}}\right)=W \cdot \hat{\pi}^{\prime}\left(I_{S_{+}}\right)
$$

where $W$ is unitary and

$$
W=U_{1} \otimes V_{\pi^{1}} \oplus U_{2} \otimes V_{\pi^{2}} \oplus \ldots \oplus U_{\infty} \otimes V_{\pi^{\infty}}
$$

for $U_{i} \in \mathscr{B}\left(\mathscr{H}_{i}\right)$ and $V_{\pi^{k}}=\bigoplus_{\alpha} e^{\imath \theta(k, \alpha)} \mathbb{1}_{\pi^{k, \alpha}}, \theta(k, \alpha) \in \mathbb{R}$. Defining $\bar{V}_{\pi^{k}}:=$ $\bigoplus_{\alpha} e^{-1 / 2 \theta(k, \alpha)} \mathbb{1}_{\pi^{k, \alpha}}, \bar{V}:=\mathbb{1}_{1} \otimes \bar{V}_{\pi^{1}} \oplus \ldots \oplus \mathbb{1}_{\infty} \otimes \bar{V}_{\pi^{\infty}}$ and conjugating both sides of (AII.5) by $\bar{V}$, we are reduced to the case of

$$
\hat{\pi}\left(I_{S_{+}}\right)=W^{\prime} \hat{\pi}^{\prime}\left(I_{S_{+}}\right),
$$

where $W^{\prime}=U_{1} \otimes \mathbb{1}_{\pi^{1}} \oplus \ldots \oplus U_{\infty} \otimes U_{\pi^{\infty}}$. Let $E_{\imath}$ be the orthogonal projection on $\mathscr{H}_{i} \otimes \mathscr{H}_{\pi^{i}}$ and set $\hat{\pi}\left(I_{S_{+}}\right)_{i}:=E_{i} \hat{\pi}\left(I_{S_{+}}\right) E_{i}$.

Since $\hat{\pi}\left(I_{S_{+}}\right)=\hat{\pi}\left(I_{S_{+}}\right)_{1} \oplus \ldots \oplus \hat{\pi}\left(I_{S_{+}}\right)_{\infty}$ it is sufficient to check that each

$$
\hat{\pi}\left(I_{S_{+}}\right)_{i}=\left(U_{i} \otimes \mathbb{1}_{\pi^{2}}\right) \hat{J}_{\pi^{2}}\left(e^{i}\right) \quad i=1,2, \ldots \infty
$$

is unitarily equivalent to $\hat{J}_{\pi^{i}}\left(e^{\imath}\right)$. Squaring both sides of (AII.7) and using the fact that

$$
\hat{\pi}\left(I_{S_{+}}\right)_{i}^{2}=\hat{J}_{\pi^{i}}\left(e^{i}\right)^{2}=\mathbb{1}_{\mathscr{H}_{\imath} \otimes \mathscr{H}_{\pi^{i}}},
$$

one sees easily that

$$
U_{i} J\left(e^{\imath}\right) U_{i} J\left(e^{\imath}\right)=\mathbb{1}_{\mathscr{H}_{\imath}}
$$

where $J\left(e^{i}\right)$ is the conjugation on $\mathscr{H}_{2}$ associated to $e^{i}$. If $i<\infty$, Eq. (AII.8) is $U_{i} \cdot \bar{U}_{i}=\mathbb{1}_{\mathscr{H}_{i}}$, where the bar means complex conjugation. Hence $U_{i}$ is a symmetric matrix and can be diagonalized by means of an orthogonal transformation $R_{i}$ :

$$
R_{i} U_{i} R_{i}^{t}=D_{i}
$$

where $D_{\imath}$ is diagonal. Conjugating both sides of (AII.6) by $R_{i} \otimes \mathbb{1}_{\pi^{i}}$ we see that

$$
\left(R_{\otimes} \mathbb{1}_{\pi^{i}}\right) \hat{\pi}\left(I_{S_{+}}\right)_{i}\left(R_{i} \otimes \mathbb{1}_{\pi^{\imath}}\right)^{*}=\left(D_{i} \otimes \mathbb{1}_{\pi^{i}}\right) \hat{J}_{\pi^{i}}\left(e^{i}\right)
$$

and we get rid of the remaining phase factors just as we did in Eqs. (AII.5), (AII.6).

If $i=\infty$, then $U_{\infty}$ has the spectral decomposition

$$
U_{\infty}=\int_{0}^{1} e^{2 \pi i \lambda} d E(\lambda)
$$

and in this case Eq. (AII.8) implies that $E(\lambda)$ commutes with $J\left(e^{\infty}\right)$ for all $\lambda$. We define

$$
W_{\infty}=\int_{0}^{1} e^{-\imath \pi \lambda} d E(\lambda), \quad W_{\infty}^{2}=U_{\infty}^{*}
$$

and obviously,

$$
J\left(e^{\infty}\right) W_{\infty} J\left(e^{\infty}\right)=W_{\infty}^{*}
$$


Hence conjugating both sides of (AII.7) by $W_{\infty} \otimes \mathbb{1}_{\pi^{\infty}}$ we obtain

$$
\begin{aligned}
& \left(W_{\infty} \otimes \mathbb{1}_{\infty}\right) \hat{\pi}\left(I_{S_{+}}\right)_{\infty}\left(W_{\infty}^{*} \otimes \mathbb{1}_{\infty}\right) \\
& \quad=\left(W_{\infty} \otimes \mathbb{1}_{\infty}\right)\left(U_{\infty} \otimes \mathbb{1}_{\infty}\right) \hat{J}_{\pi \infty}\left(e^{\infty}\right)\left(W_{\infty} \otimes \mathbb{1}_{\pi^{\infty}}\right) \\
& \quad=\left(W_{\infty} U_{\infty} W_{\infty} \otimes \mathbb{1}_{\pi^{\infty}}\right) \hat{J}_{\pi^{\infty}}\left(e^{\infty}\right) \\
& \quad=\hat{J}_{\pi^{\infty}}\left(e^{\infty}\right) .
\end{aligned}
$$

This completes the proof of the Lemma.

Proof of Theorem 2.26. Parts (i)-(iii) follow immediately from Lemma AII.1 and (iv) is an easy consequence of Lemma 2.22, we omit the proof.

\section{Appendix III. Proof of Theorem 4.42}

Proof of Theorem 4.42. Ad (i). It follows from Theorem 2.19 that the modular conjugation $J_{K}$ of $\mathscr{C}(K)$ implements antiunitarily the inversion $I_{K}$ on $\mathscr{H}$. Hence $j_{K} \circ \alpha_{h^{-1}}(\mathscr{A}(J))=\mathscr{f}\left(I_{K} \cdot h^{-1} \cdot J\right)$ and the right-hand side of Eq. (4.21) is welldefined. Since $\rho=\left\{\rho_{J}\right\}_{J \subset S^{1}}$ is a representation of $\mathscr{A}_{0}$, it follows at once that $\bar{\rho}^{K, h}=\left\{\bar{\rho}_{J}^{K, h}\right\}_{J \subset S^{1}}$ satisfies property (i) of Definition 4.1; properties (ii) and (iii) of Definition 4.1 follow from point (iii) of the theorem which will be proven below.

If $A \in \mathscr{A}\left(h \cdot I_{K} \cdot I^{\prime}\right)$ then

$$
\bar{\rho}_{h \cdot I_{k} \cdot I^{\prime}}^{K, h}(A)=\alpha_{j} \circ j_{K} \circ \rho_{I^{\prime}} \circ j_{K} \circ \alpha_{h^{-1}}(A)=A,
$$

since $\left.\rho_{I^{\prime}}\right|_{\mathscr{C}\left(I^{\prime}\right)}=\left.\mathrm{id}\right|_{\mathscr{C}\left(I^{\prime}\right)}$. It follows that $\bar{\rho}^{K, h}$ is localized in $h \cdot I_{K} \cdot I$.

Finally, local irreducibility follows from

$$
\begin{aligned}
& \bar{\rho}_{h \cdot h}^{K, h} I_{k}\left(\mathscr{A}\left(h \cdot I_{K} \cdot I\right)\right)^{\prime} \cap \mathscr{A}\left(h \cdot I_{K} \cdot I\right) \\
& \quad=\alpha_{h} \circ j_{K} \circ \rho_{I} j_{K} \circ \alpha_{h^{-1}}\left(\mathscr{A}\left(h \cdot I_{k} \cdot I\right)\right)^{\prime} \cap \mathscr{A}\left(h \cdot I_{K} \cdot I\right) \\
& \quad=\alpha_{h} \circ j_{K} \circ \rho_{I}(\mathscr{C}(I))^{\prime} \cap \mathscr{A}\left(h \cdot I_{K} \cdot I\right) \\
& \quad \cong \rho_{I}(\mathscr{A}(I))^{\prime} \cap j_{K} \circ \alpha_{h^{-1}}\left(\mathscr{A}\left(h \cdot I_{K} \cdot I\right)\right) \\
& \quad=\rho_{I}(\mathscr{A}(I))^{\prime} \cap \mathscr{A}(I)
\end{aligned}
$$

Ad (ii). By definition,

$$
\begin{aligned}
& \bar{\rho}^{K, h}(A)=\alpha_{h} \circ j_{K} \circ \rho \circ j_{K} \circ \alpha_{h^{-1}}(A), \quad A \in \mathscr{A}_{0}, \\
& \bar{\rho}^{K^{\prime}, h^{\prime}}(A)=\alpha_{h^{\prime}} \circ j_{k^{\prime}} \circ \rho \circ j_{K^{\prime}} \circ \alpha_{h^{-1}}(A), \quad A \in \mathscr{C}_{0} .
\end{aligned}
$$

An easy calculation shows that

$$
\bar{\rho}^{K, h}(A)=\operatorname{ad}\left[\alpha_{h} \circ j_{K}\left(\pi_{\rho}^{M}(\tilde{s})\right) \pi_{0}^{M}\left(h \cdot I_{K} \cdot I_{K^{\prime}} \cdot h^{-1}\right)\right] \bar{\rho}^{K^{\prime}, h^{\prime}}(A),
$$

where $\tilde{s} \in \widetilde{P S U}(1 ; 1)$ is chosen in such a way that $s=I_{K} h^{-1} h^{\prime} I_{K^{\prime}} \in P S U(1 ; 1)$.

Ad (iii). Plugging in the left-hand side of Eq.(4.22), the definitions of $\bar{\rho}_{g^{-1} J^{K}}^{K, h}(A)$ and of $\pi_{\tilde{\rho}^{K, h}}^{M}(\tilde{g})$, we obtain the right-hand side by direct computation, using the covariance of $\rho$.

Ad (iv). Let $h \in P S U(1 ; 1)$ be chosen in such a way that $h \cdot I_{J} \cdot I=I$. By Lemma 2.1 of ref. [33], it is sufficient to show the existence of a unitary implementing $\rho_{J}$ on 
$\mathscr{b}(J)$ such that $j_{J}\left(u^{*}\right)$ implements $\bar{\rho}_{J}^{J, h}$ on $\mathscr{C}(J)$. Since $\rho$ is localized in the interval $I$, the shifted representation

$$
\rho_{I_{J} \cdot h \cdot I_{J}}(A)=\operatorname{ad}\left[\Gamma_{\rho}\left(\operatorname{ad}_{I_{J}}(\tilde{h})\right)\right] \circ \rho(A), \quad A \in \mathscr{b}_{0}
$$

is localized in $I_{J} \cdot h \cdot I_{J} \cdot I=I_{J} \cdot I$. In this last equation, ad $I_{J}$ denotes the unique extension to $\widetilde{P S U}(1 ; 1)$ of the automorphism $h \mapsto I_{J} h I_{J}$ of $P S U(1 ; 1)$. If $A \in \mathscr{b}\left(I_{J} I^{\prime}\right)$ it follows from Eq. (AIII.1) that

$$
\begin{aligned}
\rho_{I_{J} \cdot I^{\prime}}(A) & =\operatorname{ad}\left[\Gamma_{\rho}\left(\operatorname{ad}_{I_{J}}(\tilde{h})\right)^{*}\right] \circ \rho_{I_{J} \cdot h \cdot I_{J}, I_{J} \cdot I^{\prime}}(A) \\
& =\operatorname{ad}\left[\Gamma_{\rho}\left(\operatorname{ad}_{I_{J}}(\tilde{h})\right)^{*}\right](A) .
\end{aligned}
$$

Since $I_{J} I^{\prime} \supseteq I_{J} \cdot J^{\prime}=J$ we may choose $u=\Gamma_{\rho}\left(\operatorname{ad}_{I_{J}}(\tilde{h})\right)^{*}$.

Moreover

$$
j_{J}\left(u^{*}\right)=j_{J}\left(\Gamma_{\rho}\left(\operatorname{ad}_{I_{J}}(\tilde{h})\right)\right)=j_{J} \circ \alpha_{I_{J} \cdot h \cdot I_{J}}\left(\Gamma_{\rho}\left(\operatorname{ad}_{I_{J}}\left(\tilde{h}^{-1}\right)\right)^{*}\right),
$$

by the cocycle identity for $\Gamma_{\rho}(\cdot)$; and

$$
\begin{aligned}
j_{J} \circ \alpha_{I_{J} \cdot h \cdot I_{J}}\left(\Gamma_{\rho}\left(\operatorname{ad}_{I_{J}}\left(\tilde{h}^{-1}\right)\right)^{*}\right) & =\alpha_{h} \circ j_{J}\left(\Gamma_{\rho}\left(\operatorname{ad}_{I_{J}}\left(\tilde{h}^{-1}\right)\right)\right)^{*}, \\
& =\left[\alpha_{h} \circ j_{J}\left(\pi_{0}^{M}\left(I_{J} \cdot h^{-1} \cdot I_{J}\right) \pi_{\rho}^{M}\left(\operatorname{ad}_{I_{J}}(\tilde{h})\right)\right)\right]^{*} \\
& =\left[\pi_{0}^{M}\left(h^{-1}\right) \alpha_{h} \circ j_{J}\left(\pi_{\rho}^{M}\left(\operatorname{ad}_{I_{J}}(\tilde{h})\right)\right)\right]^{*} \\
& =\Gamma_{\bar{\rho}} J, h\left(\tilde{h}^{-1}\right)^{*},
\end{aligned}
$$

by definition of $\pi_{\bar{\rho}, h}^{M}$.

Since the representation $\bar{\rho}^{J, h}$ is localized in $h \cdot I_{J} \cdot I$, it follows that the shifted representation

$$
\bar{\rho}_{h^{-1}}^{J, h}=\operatorname{ad} \Gamma_{\bar{\rho}^{J, h}}\left(\tilde{h}^{-1}\right) \circ \bar{\rho}^{J, h}
$$

is localized in $I_{J} \cdot I$. Hence on $\mathscr{A}\left(I_{J} \cdot I^{\prime}\right)$,

$$
A=\bar{\rho}_{h^{-1}, I_{J} I^{\prime}}^{J, h}(A)=\operatorname{ad} \Gamma_{\bar{\rho} J, h}\left(\tilde{h}^{-1}\right) \bar{\rho}_{I_{J} \cdot I^{\prime}}^{J, h}(A),
$$

$A \in \mathscr{b}\left(I_{J} I^{\prime}\right)$. This means that

$$
\begin{aligned}
\operatorname{ad} j_{J}\left(u^{*}\right)(A) & =\operatorname{ad}\left[\Gamma_{\bar{\rho} J, h}\left(\tilde{h}^{-1}\right)^{*}\right](A) \\
& =\bar{\rho}_{I_{J} I^{\prime}}^{J, h}(A), \quad \forall A \in \mathscr{A}\left(I_{J} I^{\prime}\right),
\end{aligned}
$$

so that $j_{J}\left(u^{*}\right)$ implements $\bar{\rho}^{J, h}$ on $\mathscr{A}\left(I_{J} \cdot I^{\prime}\right) \supseteq \mathscr{A}(J)$. This completes the proof of the theorem.

Acknowledgements. We are indebted to G. Felder, P. de la Harpe and, especially, to T. Kerler for many very helpful discussions and exchanges of ideas. We thank D. Buchholz, D. Kazhdan and A. Wassermann for valuable conversations and useful information. 


\section{References}

1. Belavin, A.A., Polyakov, A.M., Zamolodchikov, A.B.: Infinite conformal symmetry in twodimensional quantum field theory. Nucl. Phys. B 241, 333-380 (1984); For earlier work, see, e.g., Ferrara, S., Grillo, A.F., Gatto, R.: Conformal algebra in two space-time dimensions and the Thirring model. Il Nuovo Cimento 12A, 959-968 (1972); Fubini, S., Hanson, A.J., Jackiw, R.: New approach to field theory. Phys. Rev. D7, 1732-1760 (1973); Schroer, B.: A trip to scaling land. In: V Brazilian Symposium on Theoretical Physics, vol. 1, E. Ferreira (ed.), Rio de Janeiro: Livro Tecnicos E Cientificos Editora S.A., 1975; Lüscher, M., Mack, G.: unpubl. manuscript, 1976

2. Polyakov, A.M.: Conformal symmetry of critical fluctuations, JETP Lett.12, 381-383 (1970); Parisi, G., Peliti, L.: Calculation of critical indices, Lettere al Nuovo Cimento 2 no. 13, 627-628 (1971); For a review see: Cardy, J.: Conformal invariance, In: Phase transitions and critical phenomena 11, Domb, C., Lebowitz, J.L., eds., London: Academic Press, 1986, pp. 55-126

3. See, e.g., Fröhlich, J., Studer, U.: Incompressible quantum fluids, gauge-invariance and current algebra. In: New Symmetry Principles in Quantum Field Theory, Cargèse 1991, Plenum Press (to appear)

4. Fendley, P., Saleur, H.: Boston and Yale preprint BUHEP-92-15

5. Affleck, I.: A current algebra approach to the Kondo effect. Nucl. Phys. B 336, 517-532 (1990)

6. See, e.g., Green, M.B., Schwarz, J.H., Witten, E.: Superstring Theory. Vol. I, II, London, New York: Cambridge Univ. Press, 1987; Superstrings '89, Green M. et al. (eds.) Singapore: World Scientific, 1990

7. Goddard, P., Olive, D.: Kac-Moody and Virasoro algebras in relation to quantum physics. Int. J. Mod. Phys. A 1 no. 2, 303-414 (1986)

8. Friedan, D., Schenker, S.: The analytic geometry of two-dimensional conformal field theory. Nucl. Phys. B 281, 509-545 (1987)

9. Moore, G., Seiberg, N.: Classical and quantum conformal field theory. Commun. Math. Phys. 123, 177-254 (1989); Kerler, T.: Non-Tannakian Categories in quantum field theory. In: New Symmetry Principles in Quantum Field Theory, Cargèse 1991, Plenum Press, to appear; Ph.D. thesis, ETH 1992; and ref. [69]

10. Jones, V.: Index for subfactors. Invent. Math. 72, 1-25 (1983)

11. Felder, G.: BRST approach to minimal models. Nucl. Phys. B 241, 333-380 (1984); Felder, G., Wieczerkowski, C.: Topological Representations of the Quantum Group $U_{q}\left(s l_{2}\right)$. Commun. Math. Phys. 138, 583-605 (1991); Kerler, T.: in ref. [9]

12. Haag, R.: Local quantum field theory, In: Texts and Monographs in Physics, Berlin, Heidelberg, New York: Springer 1992

13. Doplicher, S., Haag, R., Roberts, J.: Local observables and particle statistics I, Commun. Math. Phys. 23, 199-230 (1971)

14. Doplicher, S., Haag, R., Roberts, J.: Local observables and particle statistics II. Commun. Math. Phys. 35, 49-85 (1974)

15. Buchholz, D., Fredenhagen, K.: Locality and the structure of particle states. Commun. Math. Phys. 84, 1-54 (1982)

16. Buchholz, D.: On particles, infraparticles and the problem of asymptotic completeness, VIIIth International Congress on Mathematical Physics, Marseille 1986, Mebkhout, M., Sénéor, R. (eds.), Singapore: World Scientific 1987; see also: Fröhlich, J., Morchio, G., Strocchi, F.: Charged sectors and scattering states in quantum electrodynamics. Ann. Phys. (N.Y.) 119, 241-284 (1979)

17. Buchholz, D., Doplicher, S., Longo, R.: On Noether's theorem in quantum field theory. Ann. Phys. (N.Y.) 170, 1-17 (1986)

18. Buchholz, D., Doplicher, S., Longo, R., Roberts, J.: A new look at Goldstone's theorem. Desy preprint 92-057, April 1992

19. Haag, R., Kastler, D.: An algebraic approach to quantum field theory. J. Math. Phys. 5, 848-861 (1964)

20. Doplicher, S., Roberts, J.: Why there is a field algebra with a compact gauge group describing the superselection structure in particle physics. Commun. Math. Phys. 131, 51-107 (1990)

21. Leinaas, M., Myrheim, J.: On the theory of identical particles. Il Nuovo Cimento 37B, 1-23 (1977)

22. Fredenhagen, K., Rehren, K., Schroer, B.: Superselection sectors with braid group statistics and exchange algebras, I. Commun. Math. Phys. 125, 201-226 (1989) 
23. Fröhlich, J., Gabbiani, F., Marchetti, P.-A.: Superselection structure and statistics in threedimensional local quantum field theory. In: Proc. $12^{\text {th }}$ John Hopkins Workshop on current problems in particle theory. Lusanna, G. (ed.) Singapore: World Scientific 1989

24. Fröhlich, J., Gabbiani, F., Marchetti, P.-A.: Braid statistics in three-dimensional local quantum theory. In: The algebraic theory of superselection sectors. Introduction and recent results, Singapore: World Scientific 1990

25. Fröhlich, J., Gabbiani, F.: Braid statistics in local quantum theory. Rev. Math. Phys. 2, 251-353 (1990)

26. Mack, G.: Introduction to conformal invariant quantum field theory in two and more dimensions. In: Non-perturbative quantum field theory. G. t' Hooft et al. (eds.) Plenum Press, 1988. Proceedings of the Cargèse Summer School 1987

27. Eguchi, T., Ooguri, H., Taormina, A., Yang, S.: Superconformal algebras and string compactification on manifolds with $S U(n)$ holonomy. Nucl. Phys. B 315, 193-221 (1989)

28. Warner, N.P.: Lectures on $N=2$ superconformal theories and singularity theory. In: Superstrings ' 89 Green, M. et al. (eds.) Singapore: World Scientific 1990

29. Wassermann, A.: Subfactors arising from positive-energy representations of some infinite dimensional groups, preliminary notes

30. Buchholz, D., Mack, G., Todorov, I.: The current algebra on the circle as a germ of local field theories. Nucl. Phys. B (Proc. Suppl.) 5B, 20-56 (1988)

31. Buchholz, D., Schulz-Mirbach, H.: Haag duality in conformal field theory. Rev. Math. Phys. 2, 105-125 (1990)

32. Fredenhagen, K.: Generalization of the theory of superselection sectors. In: The algebraic theory of superselection sectors. Introduction and recent results. Singapore: World Scientific 1990

33. Guido, D., Longo, R.: Relativistic invariance and charge conjugation in quantum field theory. (Preprint of the University of Rome 'Tor Vergata', 1992)

34. Borchers, H.J.: The CPT theorem in two-dimensional theories of local observables. Commun. Math. Phys. 143, 315-332 (1992)

35. Borchers, H.J.: On the converse of the Reeh-Schlieder theorem. Commun. Math. Phys. 10, 269-273 (1968)

36. Takesaki, M.: Tomita's theory of modular Hilbert algebras and its applications. Lecture Notes in Math. 128 (1970)

37. Bisognano, J., Wichmann, E.: On the duality condition for a Hermitian scalar field. J. Math. Phys. 16, 985-1007 (1975); J. Math. Phys. 17, 303-321 (1976)

38. Borchers, H.J.: A remark on a theorem of B. Misra. Commun Math. Phys. 4 315-323 (1967)

39. D'Antoni, C.: Technical properties of the quasi-local algebra. In: The algebraic theory of superselection sectors. Introduction and recent results. Singapore: World Scientific 1990

40. Bargmann, V.: Irreducible unitary representations of the Lorentz group. Ann. Math. 48, 568-640 (1947)

41. Lang, S.: $S L(2 ; \mathbb{R})$, Reading, MA: Addison-Wesley 1975

42. Pressley, A., Segal, G.: Loop groups. Oxford: Clarendon Press 1988

43. Driessler, W.: Comments on light-like translations and applications in relativistic quantum field theory. Commun. Math. Phys. 44, 133-141 (1975)

44. Connes, A.: Une classification des facteurs de type III. Ann. Scient. Ec. Norm. Sup. 4e Série, 6 (1973) pp. 133-252

45. Maison, D.: Eine Bemerkung zu Clustereigenschaften. Commun. Math. Phys. 10, $48-51$ (1968)

46. Buchholz, D., D'Antoni, C., Fredenhagen, K.: The universal structure of local algebras. Commun. Math. Phys. 111, 123-135 (1987)

47. Kac, V.: Infinite dimensional Lie algebras. $3^{\text {rd }}$ ed. Cambridge: Cambridge Univ. Press 1990

48. Borchers, H.J., Schumann, R.: A nuclearity condition for charged states. Lett. Math. Phys. 23, 65-77 (1991)

49. Buchholz, D., Yngvason, J.: Generalized Nuclearity conditions and the split property in quantum field theory. Lett. Math. Phys. 23, 159-167 (1991)

50. Doplicher, S.: Local aspects of superselection rules. Commun. Math. Phys. 85, 73-86 (1982)

51. Araki, H.: Von Neumann algebras of local observables for free scalar field. J. Math. Phys. 5, 1-13 (1964)

52. Eckmann, J.P., Osterwalder, K.: An application of Tomita's theory of modular Hilbert algebras: Duality for free Bose fields. J. Funct. Anal. 13, 1-12 (1973) 
53. Hislop, P.D., Longo, R.: Modular structure of the local algebras associated with the massless scalar field. Commun. Math. Phys. 84, 71-85 (1982)

54. Rigotti, C.: Remarks on the modular operators and local observables. Commun. Math. Phys. 61, 267-273 (1978)

55. Wigner, E.: In: Group theory and its applications to the quantum mechanics of atomic spectra. New York: Academic Press 1959

56. Landau, L., Wichmann, J.: On the translation invariance of local internal symmetries. J. Math. Phys. 11, 306-311 (1970)

57. Hamilton, R.S.: The inverse function theorem of Nash and Moser. Bull. Am. Phys. Soc. (New Series) 7, 65-222 (1982)

58. Segal, G.: Unitary representations of some infinite dimensional groups. Commun. Math. Phys. 80, 301-342 (1981)

59. Mickelsson, J.: Kac-Moody groups, topology of the Dirac determinant bundle and fermionization. Commun. Math. Phys. 110, 173-183 (1987)

60. Mickelsson, J.: In: Current algebras and groups. New York: Plenum Press 1989

61. Goodman, R., Wallach, N.: Structure and unitary cocycle representations of loop groups and the group of diffeomorphisms of the circle. J. für reine u. angew. Math. 347, 69-133 (1984)

62. Kac, V., Wakimoto, M.: Modular and conformal invariance constraints in the representation theory of affine algebras. Adv. Math. 70, 156-236 (1986)

63. Dixmier, J.: Les algèbres d'opérateurs dans l'espace Hilbertien. Paris: Gauthier-Villars 1956

64. Doplicher, S., Haag, R., Roberts, J.: Fields, Observables and Gauge transformations, II. Commun. Math. Phys. 15, 173-200 (1969)

65. Roberts, J.: Spontaneously broken gauge symmetries and superselection rules. In: Proceedings of the international school of mathematical physics, Camerino 1974, Gallavotti, G., (ed.) Università di Camerino 1976

66. Roberts, J.: Some applications of dilatation invariance to structural questions in the theory of local observables. Commun. Math. Phys. 37, 273-286 (1974)

67. Longo, R.: Index of subfactors and statistics of quantum fields, I. Commun. Math. Phys. 126, 217-247 (1989)

68. Longo, R.: Index of subfactors and statistics of quantum fields, II. Commun. Math Phys. 130, 285-309 (1990)

69. Fröhlich, J., Kerler, T.: On the rôle of quantum groups in low dimensional local quantum field theories. Preliminary version, July 1991, to appear in Springer Lect. Notes in Math.

70. Rehren, K.: Braid group statistics and their superselection rules. In: The algebraic theory of superselection sectors. Introduction and recent results, Singapore: World Scientific 1990

71. Fuchs, J.: Quantum dimensions, CERN preprint 6/56/91, July 1991

72. Wenzl, H.: Hecke algebras of type $A_{n}$ and subfactors. Inv. Math. 92, 349-383 (1988)

73. Wenzl, H.: Quantum groups and subfactors of type $B, C$ and $D$. Commun. Math Phys. 133, 383-432 (1990)

74. Alvarez, A., Singer, I.M., Windey, P.: The supersymmetric $\sigma$-model and the geometry of the Weyl-Kac character fomula. Preprint UCB-PTH 91/37

75. Strătilă, S., Zsidǒ, L.: In: Lectures on von Neumann algebras. England: Abacus Press, 1979

76. Mackey, G.: In: The theory of unitary group representation. $3^{\text {rd }}$ ed. Chicago, IL: The Univ. of Chicago Press, 1976

77. Fredenhagen, K., Rehren, K.-H., Schroer, B.: Superselection Sectors with Braid Group Statistics and Exchange Algebras, II. SFB 228, Preprint no. 10, Berlin, April 1992. Wiesbrock, H.-W.: Conformal Quantum Field Theory and Half- sided Modular Inclusions of von NeumannAlgebras. SFB 288, Preprint no. 35.

78. Goodman, R., Wallach, N.: Projective unitary Positive-energy representations of $\operatorname{Diff}\left(S^{1}\right)$. J. Funct. Anal. 63, 299-321 (1985)

79. Feigin, B.L., Fuchs, D.B.: Verma modules over the Virasoro algebra. In: Lecture Notes in Mathematics $\mathrm{n}^{\circ}$ 1060, Berlin, Heidelberg, New York: Springer 1984, pp. 230-245

80. Mack, G., Schomerus, V.: Conformal Field Algebras with Quantum Symmetry from the Theory of Superselection Sectors. Commun. Math. Phys. 134, 139-196 (1990). Fuchs, J., Ganchev, A., Vecsernyes, P.: Level 1 WZW superselection sectors. Commun. Math. Phys. 146, 553 (1992) 